\title{
Understanding Falls Risk Screening Practices and Potential for Electronic Health Record Data-Driven Falls Risk Identification in Select West Virginia Primary Care Centers
}

Adam Baus

Follow this and additional works at: https://researchrepository.wvu.edu/etd

\author{
Recommended Citation \\ Baus, Adam, "Understanding Falls Risk Screening Practices and Potential for Electronic Health Record \\ Data-Driven Falls Risk Identification in Select West Virginia Primary Care Centers" (2015). Graduate \\ Theses, Dissertations, and Problem Reports. 5166. \\ https://researchrepository.wvu.edu/etd/5166
}

This Dissertation is protected by copyright and/or related rights. It has been brought to you by the The Research Repository @ WVU with permission from the rights-holder(s). You are free to use this Dissertation in any way that is permitted by the copyright and related rights legislation that applies to your use. For other uses you must obtain permission from the rights-holder(s) directly, unless additional rights are indicated by a Creative Commons license in the record and/ or on the work itself. This Dissertation has been accepted for inclusion in WVU Graduate Theses, Dissertations, and Problem Reports collection by an authorized administrator of The Research Repository @ WVU.

For more information, please contact researchrepository@mail.wvu.edu. 
Understanding Falls Risk Screening Practices and Potential for Electronic Health Record Data-Driven Falls Risk Identification in Select West Virginia Primary Care Centers

\author{
Adam Baus \\ Dissertation submitted to the \\ School of Public Health \\ at West Virginia University \\ in partial fulfillment of the requirements for the degree of \\ Doctor of Philosophy \\ in \\ Public Health Sciences
}

\author{
Jeffrey Coben, MD, Chair \\ Keith Zullig, PhD \\ Dustin Long, $\mathbf{P h D}$ \\ Charles Mullett, MD, PhD \\ Cecil Pollard, MA \\ Henry Taylor, MD, MPH
}

Department of Social and Behavioral Sciences

\title{
Morgantown, West Virginia 2015
}

Keywords: Unintentional Falls, Electronic Health Record, Screening, Primary Care 


\title{
ABSTRACT \\ Understanding Falls Risk Screening Practices and Potential for Electronic Health Record Data-Driven Falls Risk Identification in Select West Virginia Primary Care Centers
}

\begin{abstract}
Adam Baus
Unintentional falls among older adults are a complex public health problem both nationally and in West Virginia. Nationally, nearly $40 \%$ of community-dwelling adults age 65 and older fall at least once a year, making unintentional falls the leading cause of both fatal and non-fatal injuries among this age group. This problem is especially relevant to West Virginia, which has a population ageing faster on average than the rest of the nation. Identifying falls risk in the primary care setting poses a serious challenge. Currently, the Timed Get-Up-and-Go test is the only recommended screening tool for determining risk. However, nationally this test is completed only $30-37 \%$ of the time. Use of electronic health record data as clinical decision support in identifying at-risk patients may help alleviate this problem. However, to date there have been no published studies on using electronic health record data as clinical decision support in the identification of this particular population. This presents opportunity to contribute to the fields of falls prevention and health informatics through novel use of electronic health record data. That stated, this research is designed to: 1) develop an understanding of current falls risk screening practices, facilitators, and barriers to screening in select West Virginia primary care centers; 2) assess the capture of falls risk data and the quality of those data to help facilitate identification of at-risk patients; and 3) build an internally validated model for using electronic health record data for identification of at-risk patients. Through focus group discussions with primary care partners, we find a significant lack of readiness to innovatively use routinely collected data for population health management for falls prevention. The topic of falls risk identification is a rarely discussed topic across these sites, with accompanying low rates of screening and ad-hoc documentation. The need for enhanced team-based care, policy, and procedure surrounding falls is evident. Using de-identified electronic health record data from a sample of West Virginia primary care centers, we find that it is both feasible and worthwhile to repurpose routinely collected data to identify older adult patients at-risk for falls. Among 3,933 patients 65 and older, only 133 patients $(3.4 \%)$ have an indication in their medical records of falling. Searching the free text data was vital to finding even this low number of patients, as $33.8 \%$ were identified using free text searches. Given the focus group findings, underreporting of falls on the part of the patients and missed opportunities to learn of falls due to lack of information sharing across health care service sites are also contributing factors. Similarly, documentation of falls risk assessments were sparse with only 23 patients $(0.6 \%)$ having documentation of a falls risk assessment in their medical records at some point in the past. As with falls, locating documentation of falls risk assessments was largely dependent on semistructured and free text data. Current Procedural Terminology coding alone missed $26.1 \%$ of all falls risk assessments. Repurposing electronic health record data in a population health framework allows for concurrent examination of primary and secondary falls risk factors in a way which is sensitive to time constraints of the routine office visit, complementary to the movement toward Meaningful Use, while providing opportunity to bolster low screening rates.
\end{abstract}




\section{DEDICATION}

To my wife, Angela, and our children, Samuel and Grace. 


\section{ACKNOWLEDGMENTS}

I write my sincere thanks to my Dissertation Committee, a group of individuals who took the time and energy to work with me and treat me like a colleague. Dr. Jeffrey Coben, you were instrumental in concept development and implementation. Your pragmatic advice and teaching has made this dissertation possible. Dr. Keith Zullig, I thank you for not only being a guide in this work but also having the trust in allowing me to be the first part-time student in this program. Dr. Dustin Long, I thank you for your leadership and vision for the statistical analysis in this research and for your support overall. Dr. Charles Mullett, your insight into the approach to the dissertation and informatics were vital. Dr. Henry Taylor, your ability to teach during everyday conversation helped to keep me grounded and the research actionable. Cecil Pollard, you have been and continue to be a true mentor to me. Working with you on this, as with our work together in the Office of Health Services Research, is a privilege.

Further, I give my thanks to Dr. Geri Dino. Your encouragement, support, and partnership continue to be a gift. Thank you too for providing funds to cover the focus group transcriptions. And, my sincere gratitude to Dr. Traci Jarrett for your guidance, willingness to listen, and ability to ask challenging questions. Thank you for that. Here's to many more years of working together. 


\section{TABLE OF CONTENTS}

ABSTRACT

DEDICATION__ iii

ACKNOWLEDGMENTS _

CHAPTER 1 _ 1

1.1 Introduction and Background __ 2

1.1.1 Etiology of unintentional falls among older adults in Appalachia __ 3

1.2 Statement of the Problem __ 4

1.2.1 Application of electronic health records to clinical quality improvement _ 5

1.2.2 Free text electronic health record data _ 7

1.3 Purpose of the Current Research__ 9

CHAPTER 2 는 10

2.1 Introduction

2.2 Methods 13

2.2.1 Participants — 13

2.2.2 Measures

2.2.3 Analysis 16

2.3 Results 19

2.3.1 Current culture: perceptions of older adult patients _ 19

2.3.2 Current culture: resources 22

2.3.3 Current culture: team-based care — 27

2.3.4 Current practice: policy and procedure _ 30

2.4 Discussion 33

2.4.1 Limitations — 35

2.5 Conclusions — 35

CHAPTER 3 _ 37

3.1 Introduction 38

3.2 Methods 40

3.2.1 Participants — 43

3.2.2 Measures _ 43

3.2.3 Analysis — 45

3.3 Results 46

3.4 Discussion 54

3.4.1 Limitations — 55

3.5 Conclusions — 56

CHAPTER 4 _ 57

4.1 Introduction

4.2 Methods 59

4.2.1 Participants — 60

4.2.2 Measures__ 61

4.2.3 Analysis — 62

4.3 Results 62 
4.3.1 Model 1:priority falls risk variables

4.3.2 Model 2:extended falls risk variables

4.3.3 Model 3:combined priority and extended falls risk variables

4.4 Discussion

4.4.1 Limitations

4.5 Conclusions

CHAPTER 5

5.1 Summary 80

5.2 Significance 81

5.3 Strengths and Weaknesses 83

5.4 Future Research

5.4.1 Electronic health record data quality

5.4.2 Testing external validity of falls risk identification models

5.5 Conclusions

APPENDIX A: AIM 1 INSTITUTIONAL REVIEW BOARD APPROVAL 89

APPENDIX B: FOCUS GROUP INVITATION LETTER 90

APPENDIX C: INFORMED CONSENT FORM 91

APPENDIX D: STEADI TOOLKIT 92

APPENDIX E: PHYSICIAN QUALITY REPORTING SYSTEM MEASURE 154 - FALLS RISK ASSESSMENT 93

APPENDIX F: QUALITATIVE ANALYSIS CODEBOOK 97

APPENDIX G: AIMS 2 AND 3 INSTITUTINOAL REVIEW BOARD

APPROVAL 113

APPENDIX H: PRIORITY FALLS RISK FACTORS, LOCATIONS OF DATA EXTRACTION, AND CODING

APPENDIX I: EXPANDED FALLS RISK FACTORS, LOCATIONS OF EXTRACTION, AND CODING

APPENDIX J: FALLS RISK FACTORS, REFERENCES, AND RISK

CATEGORIES 134

APPENDIX K: AIM 3 DATA DICTIONARY 137

REFERENCES 
Chapter 1 


\section{Chapter 1}

\section{Addressing unintentional falls among older adults using health information technology}

\subsection{Introduction and Background}

Unintentional falls among older adults are a complex, formidable public health problem both nationally and in West Virginia (WV). These events often result in moderate to severe injuries such as head trauma and fractures while increasing the risk of early death (Centers for Disease Control and Prevention, 2014). Recent information from the US Preventive Services Task Force (USPSTF) highlights that nearly $40 \%$ of community-dwelling adults age 65 and older fall at least once a year, making unintentional falls the leading cause of both fatal and nonfatal injuries among this age group (Michael, et al., 2010; Centers for Disease Control and Prevention, 2014). Unintentional falls accounted for more than $70 \%$ of emergency department visits among persons age 65 and older in 2010 (Villaveces, Mutter, Owens, \& Barrett, 2013). In 2012, there were 2.4 million non-fatal emergency department visits due to falls among older adults, with approximately 722,000 of those events resulting in hospitalizations (Centers for Disease Control and Prevention, 2014). Further, recent research highlights an increased prevalence of falls among older adults (Cigolle, et al., 2015). This problem is especially relevant to $\mathrm{WV}$, which has a population ageing faster on average than the rest of the nation (Christiadi, 2010; US Census Bureau, 2012). Further, poor health outcomes and complications following falls are exacerbated by various comorbidities prevalent among older adults (Carpenter, Scheatzle, D'Antonio, Ricci, \& Coben, 2009). Direct medical costs associated with these injuries were about $\$ 19.2$ billion in 2000 (Michael, et al., 2010), approximately \$30 billion in 2012 (Centers for Disease Control and Prevention, 2014), and are projected to reach $\$ 43.8$ billion by 2020 (Michael, et al., 2010). 
From 1995 to 1997, unintentional falls were the second leading cause of injury and death among West Virginians age 65 to 74 , and the leading cause of death among those age 75 and older (West Virginia Bureau for Public Health, 2008). During that time, falls were also the most common source of injury and hospital admissions among adults age 65 and older. While a WV Healthy People 2020 plan is not yet available, national Healthy People 2020 Objectives for older adults call for a 10\% reduction in emergency department visits due to falls (baseline: 5,235.1 emergency department visits per 100,000 in 2007; target: 4,716 emergency department visits per 100,000) (US Department of Health and Human Services, 2013). Despite the severity of the problem among older adults, less than half of those who do fall tell their health care providers about having fallen, making this largely preventable problem more difficult to address (Centers for Disease Control and Prevention, 2014).

\subsubsection{Etiology of unintentional falls among older adults in Appalachia}

Primary causes of falls among older adults in Appalachia include: being age 65 or older (Whiteman, Davidov, Tadros, \& D'Angelo, 2012); tripping and slipping on surfaces within the home, especially while at home alone during the winter months (Berg, Alessio, Mills, \& Tong, 1997); use of certain high-risk medications such as those effecting the central nervous system and those used as anti-hypertensives and diuretics (Blalock, et al., 2010; Casteel, Blalock, Ferreri, Roth, \& Demby, 2011; Johnson, 1985; Richardson, Hicks, \& Walker, 2002); pre-existing history of falls, especially falls occurring in the past 12 months (Carpenter, Scheatzle, D'Antonio, Ricci, \& Coben, 2009); low vision (Freeman, Muñoz, Rubin, \& West, 2007); certain neurological and cardiovascular health conditions (Lewis, Moutoux, Slaughter, \& Bailey, 2004); and diabetes (Maurer, Burcham, \& Cheng, 2004). 


\subsection{Statement of the Problem}

Identifying community-dwelling, non-institutionalized older adults at-risk for falling poses a serious challenge. Currently, the Timed Get-Up-and-Go test is the only screening tool recommended by the USPSTF for determining falls risk (Moyer, 2012). This test is performed by observing the time it takes a person to rise from an armchair, walk 3 meters (10 feet), turn, walk back, and sit down again (Podsiadlo \& Richardson, 1991). On average, a healthy adult 60 years of age or older can complete the test in less than 10 seconds. A time of 14 seconds or more is associated with high risk for falls (Bohannon, 2006). However, the Timed Get-Up-and-Go test is best considered within a larger battery of tests to more definitively measure physical mobility (Lindsay, James, \& Kippen, 2004; Piva, Fitzgerald, Irrgang, Bouzubar, \& Starz, 2004), and is dependent on clinicians using standard procedures and equipment (Siggeirsdóttir, Akranes, Jónsson Jr., \& Iwarsson, 2002). Furthermore, while it is possible to complete the test in less than a minute, this additional task, much like other preventive screenings, can be challenging to incorporate into brief office visits given the complex health needs of older patients (Boyd, et al., 2005; Jones, Ghosh, Horn, Smith, \& Vogt, 2011). National studies suggest that physicians caring for older adults provide recommended fall screening only 30-37\% of the time (Hayden, et al., 2004). This contributes to a more reactive rather than proactive approach to care, and contributes to overall low levels of quality indicators among older patients (Wenger, et al., 2003).

The challenges in incorporating falls risk screening into primary care culminate in a problem of missed opportunity for screening, counseling, intervention, and ultimately prevention of falls among older adults. Given the need for regular, ongoing falls risk screening within a challenging primary care environment, exploring use of electronic health record (EHR) data as clinical decision support in identifying at-risk patients may help alleviate this problem. Clinical 
decision support refers to a system or process designed to present the health care team with information to enhance the quality of patient care (American Medical Association, 2013; Malack, 2012). EHR data have been found to be viable clinical decision support in identifying patients with and at-risk for some chronic health conditions (Baus, Wood, Pollard, Summerfield, \& White, 2013; Hanna, Anderson, \& Maddox, 2005; Nichols, et al., 2012; Terry, et al., 2010). In a study using EHR data to identify patients with hypertension, a statistically significant increase in cases was detected based on combined use of diagnostic and free-text coding (mean =1,256.1, 95\% CI 1,232.3-1,279.7) compared to diagnostic coding alone (mean $=1,174.5,95 \% \mathrm{CI}$ 1,150.5-1,198.3) (Baus, Hendryx, \& Pollard, 2012). However, to date there have been no published studies on using EHR data as clinical decision support in the identification of older adults at-risk for falls. This presents opportunity to contribute to the fields of falls prevention and health informatics through novel use of EHR data to identify at-risk patients.

\subsubsection{Application of electronic health records to clinical quality improvement}

The potential benefits of EHRs for clinical quality improvement in primary care are well established. EHRs are intended to facilitate efficient, secure, and accurate data sharing across care sites, offer decision support for patient care, improve the management of medical information, reduce health disparities, and help improve patient care at reduced cost (Hanna, Anderson, \& Maddox, 2005; Milery \& Kukafka, 2010; Murphy, 2010; Simon, Rundall, \& Shortell, 2005; Vishwanath, Singh, \&Winkelstein, 2010). These systems are also intended to increase opportunities for outcomes research and population level surveillance in primary care settings (Dean, et al., 2009; Ethredge, 2010; Hanna, Anderson, \& Maddox, 2005; Persell, Kho, Thompson, \& Baker, 2009; Terry, et al., 2010; Weiner, Lyman, Murphy, \& Weiner, 2007). 
However, there are well-known barriers to full adoption and integration of EHRs that prevent the potential benefits of using these systems from being realized. Previous research has focused on barriers at the national and organizational levels. Common barriers are:

- lack of national standards in EHR data formats (Amatayakul, 2005; Baron R. , 2007;

Bates, 2005; Bradley, Penberthy, Devers, \& Holden, 2010; Bristol, 2005; Hanna, Anderson, \& Maddox, 2005; Miller \& Sim, 2004; Satinsky, 2004; Taylor, et al., 2005);

- lack of clinic-level readiness to adopt the EHR (Amatayakul, 2005; Himmelstein \& Woodlander, 2005; Satinsky, 2004);

- difficulties in redesigning the clinic to integrate the EHR into the office flow (Baron R., 2007; Berg M. , 1999; Berg W. , 1997; Hersh, 2002; Kuhn \& Guise, 2001; Lorenzi \& Riley, Managing change: an overview, 2000; McDonald, 1997; Vishwanath, Singh, \& Winkelstein, 2010);

- lack of clinic-level leadership to foster and help advance the EHR (Burton, Anderson, \& Kues, 2004; California Healthcare Foundation, 2003; Doolan, Bates, \& James, 2003; Lorenzi, Riley, Blyth, Southon, \& Dixon, 1997; Lorenzi \& Riley, Managing change: an overview, 2000; Satinsky, 2004; Wagner, Lee, White, Ward, \& Ornstein, 2000);

- lack of time, training and resources for care providers to be proficient in using the EHR (Bates, 2005; Gans, Kralewski, Hammons, \& Dowd, 2005; Hersh, 2002; Kristianson, Ljunggren, \& Gustafsson, 2009; May, 2005; Satinsky, 2004; Walsh, 2004).

Various federal initiatives and incentives have been implemented, beginning in the 1960s, to help spur EHR adoption and overcome the aforementioned barriers. Despite these efforts, full-adoption remains limited (Baron, Fabens, Schiffman, \& Wolf, 2005; Bristol, 2005; Gans, Kralewski, Hammons, \& Dowd, 2005; Goldschmidt, 2005; McDonald, 1997; Miller \& 
Sim, 2004; Office of the National Coordinator for Health Information Technology, 2015).

Recent federal initiatives, such as the Framework for Strategic Action, the Health Information Technology for Economic and Clinical Health Act, and the National Committee for Quality Assurance Patient-Centered Medical Home, represent more than $\$ 30$ billion in incentives for EHR adoption and use (Blumenthal \& Tavenner, 2010; Etheredge, 2010). Significant federal funding continues to be allocated to EHR adoption and meaningful use of EHR data through efforts such as the Medicare EHR incentive program (Office of the National Coordinator for Health Information Technology, 2015).

\subsubsection{Free text electronic health record data}

Research on barriers to full adoption and integration of EHRs in US primary care tends to focus on barriers at the national and organizational levels. However, an important barrier to fulluse of EHRs for patient care, tracking, quality improvement, and practice-based research that has not received much research attention is poor EHR data quality due to free text data entry. Free text data entry, as opposed to use of drop-down menus and pick-lists, results in non-standardized data that are difficult to retrieve due to coding inconsistencies, and results in reports and patient lists that are inaccurate (Benin, et al., 2005; Chan, Fowles, \& Weiner, 2010; Dean, et al., 2009; Forster, et al., 2008; Hanna, Anderson, \& Maddox, 2005; Hoff, Ottestad, Skaflotten, Bretthauer, \& Moritz, 2009; Milery \& Kukafka, 2010; Nahm, Pieper, \& Cunningham, 2008; Terry, et al., 2010; Weiner, Lyman, Murphy, \& Weiner, 2007, Wrightson, 2010). Such inaccuracy can lead to physician distrust of the data, and in-turn increased resistance to using the EMR (MaxwellDowning, 2011). Data quality as a barrier to EMR use contrasts with the previously listed national and organizational barriers in that data quality is primarily an end-user consideration. Only recently has improving the management of EHR data started to gain attention as a vital 
component in the overall success of EHR-based projects and research (Chan, Fowles, \& Weiner, 2010; Damberg, et al., 2010; Dean, et al., 2009; Farley, Dalal, Mostashari, \& Frieden, 2010; Hoff, Ottestad, Skaflotten, Bretthauer, \& Moritz, 2009; Kristianson, Ljunggren, \& Gustafsson, 2009; Pandza, 2009; Romano \& Stafford, 2011; Terry, et al., 2010; Wrightson, 2010).

Compromised data quality due to free text entries has received little research attention. There is a dearth of research into the underlying reasons why free text entry occurs. Research has instead tended to focus on development of methods for coping with free text results, such as through the use of natural language processing software to search data recorded in EHR problem lists (Friedman \& Hripcsak, 1999; Hazlehurst, et al,, 2005; Heinze, Morsch, \& Holbrook, 2001; Hersh, Campbell, Evans, \& Brownlow, 1996, Meystre \& Haug, 2005; Meystre \& Haug, 2006). Similarly, little research attention has been given to how the evolution of the medical record impacts documentation and physician interaction with the medical record. Siegler (2010) helps to fill this research gap. Through in-depth qualitative research into the history of the medical record, Siegler cautions that the structure of medical records can impact the ways in which physicians practice and document care. Siegler also cautions that the transition from paper-based to electronic-based records should be informed by the evolution of the health record during eighteenth and nineteenth century US medicine - a period marked by the introduction of more structured, standardized paper forms compared to free text, retrospectively written medical records. This structure was not well received overall, resulting in many physicians continuing to provide narrative records on the back-sides of the standardized forms to retain creativity in thought and contextual information regarding care. Siegler's work cautions that the design of modern-day EHRs, characterized with check-boxes and standardized templates, may lead to the same shortcomings in documentation. 


\subsection{Purpose of the Current Research}

This research is designed to: develop an understanding of current falls risk screening practices as well as facilitators and barriers to screening in select WV primary care centers; assess the capture of falls risk data in the EHR and the quality of those data; determine potential for use of EHR data to help facilitate identification of at-risk patients; and build an internally validated model for using EHR data for identification of at-risk patients. The resultant information, methods, and tools can help foster the Institute for Healthcare Improvement's Triple Aim of improved quality of patient-level care, improved health of patient populations, and decreased health care costs (Institute for Healthcare Improvement, 2013). That stated, the aims of this research are as follows:

Aim 1. Develop an understanding of current falls screening practices in select primary care centers, the impeding and promoting determinants to falls screening, and the potential for EHR data-based clinical decision support to be incorporated into the care setting.

Aim 2. Examine the utility of importing EHR data into an external clinical information system to systematically identify older patients at risk for falls, incorporating methods for determining the accuracy and completeness of the data. A sub-aim uses natural language processing methods to assess the potential for and value of additional falls risk information from free text or narrative data in the EHR.

Aim 3. Build and internally validate a model for case finding of older patients at-risk for falls based on EHR data for clinical decision support in the early identification of at-risk patients. 
Chapter 2 


\section{Chapter 2}

\section{Better understanding falls screening practices in select West Virginia primary care centers}

\subsection{Introduction}

Aim 1 develops an understanding of falls screening practices in select WV primary care centers, the impeding and promoting determinants to falls screening, and the potential for EHR data-based clinical decision support for falls screening to be incorporated into the care setting.

Risk factors for falls among older adults are often poorly identified in clinical practice. National studies suggest that physicians caring for older adults provide recommended falls screening only $30-37 \%$ of the time (Hayden, et al., 2004). This contributes to a reactive rather than proactive approach to care and to lower levels of quality indicators among older patients (Shires, et al., 2012; Wenger, et al., 2003). Given prior successes in applying EHR data to efforts in patient identification and research (Hanna, Anderson, \& Maddox, 2005; Okun, et al., 2013; Terry, et al., 2010), the use of EHR data as a supplemental means of identifying patients at-risk for falls has potential to support overall efforts in patient screening. This should be approached with caution, however, as numerous potential innovations in primary care have been unsuccessful because they were introduced without knowledge of provider willingness to adopt (Kaplan \& Harris-Salamone, 2009; Poses, 1999) and lacked sensitivity to the interrelation between the innovation and the organization (Berg, 2001).

Given the challenges in incorporating regular, ongoing falls risk screening in primary care, this study explores use of EHR data as clinical decision support in identifying at-risk older adults. This study aims to develop an understanding of current falls screening practices in select WV primary care centers, the impeding and promoting determinants to falls screening, and the potential for EHR data-based clinical decision support to be incorporated into the care setting. 
Understanding these issues can bolster falls risk screening and identification of at-risk patients for the purpose of falls prevention. Such clinical decision support may queue medical providers to target the Timed Get Up and Go test to specific patients and/or queue referrals for specialty care for patients identified as at-risk based on findings generated from medical records data.

Stage Theory of Organizational Change and Diffusion of Innovations Theory are the theoretical constructs guiding this aim. Taken together, these theories help identify key factors and estimate the changeability of those factors in improving falls risk screening. Stage Theory of Organizational Change is especially relevant when policies and practices of formal organizations, such as primary care centers, have been identified as environmental factors to be changed (Glanz, 2002). A change in policy, culture, and/or environmental conditions in the organization is often needed to enable the adoption, implementation, and sustainability of an innovation (Bartholomew, Parcel, Kok, Gottlieb, \& Fernandez, 2011). Innovation in primary care describes a new approach, program, or product relating to patient care. Diffusion is the process by which the innovation becomes implemented and ultimately adopted (Bush, Lord, \& Borrott, 2009). Diffusion of Innovations Theory cautions that an innovation's acceptance is dependent on an interplay of factors spanning the intended audience to the innovation itself (Fitzgerald, Ferlie, Wood, \& Hawkins, 2002; Rogers, 1983; Sanson-Fisher, 2004). In primary care, stages of acceptance, decision patterns, and change agents are at-work in the choice to accept or reject an innovation (California HealthCare Foundation, 2002; Clarke, 1999; Rogers, 1983; Sanson-Fisher, 2004). Innovations are more likely to be accepted if they clearly demonstrate a relative advantage, are compatible with existing office flows, are observable or visible to others, allow for ease in trialability, and afford opportunity for the end-user to provide 
input and refinement (California HealthCare Foundation, 2002; Harting, Rutten, Rutten, \& Kremers, 2009; Rogers, 1983; Sanson-Fisher, 2004).

This study uses focus group interviews as a qualitative application of Stage Theory of Organizational Change and Diffusion of Innovations Theory to: develop an understanding of current falls screening practices in select primary care centers; identify the impeding and promoting determinants to falls screening; and determine the potential for EHR data-based clinical decision support to be incorporated into the care setting. Focus groups are efficient for gaining insight into complex topics, allow for information gathering directly from persons who have vested interest and in-depth knowledge (Miles \& Huberman, 1994), have been effective in gathering information in primary care (Harting, Rutten, Rutten, \& Kremers, 2009; Twohig \& Putnam, 2002) and have been effectively used to study EHR adoption (Rose, et al, 2005)

The focus groups were conducted in a way which ensures confidentiality of participants and organizations. The nature of the questions does not entail personal or necessarily emotional information, and thus there was minimal risk of harm. No person was required to take part, and was given opportunity to end their participation at any time and/or have the information gleaned from talking with them removed from the results. This study was reviewed by the West Virginia University Institutional Review Board and granted exempt status (protocol number 1403223131) (Appendix A).

\subsection{Methods}

\subsubsection{Participants}

This study was carried out in rural WV primary care centers. These clinics are considered to be safety-net locations, providing care for patients in medically underserved areas of the state. Site recruitment began by contacting the administrators of the identified sites to introduce the 
study and gain approval. Given administrator approval, the physicians, nurses, and medical staff were provided information on the study and invited to participate by health center administration. See Appendix B for a copy of the focus group invitation provided to health center administrators, and Appendix $\mathrm{C}$ for a copy of the informed consent form used at the time of the focus groups. To help facilitate site recruitment, the Stopping Elderly Accidents, Deaths \& Injuries (STEADI) tool kit for health care providers (Centers for Disease Control and Prevention, 2013) was provided (Appendix D). The STEADI toolkit includes patient education materials designed to help at-risk patients understand their risks for falls, prevent falls, check their homes for safety, plus exercises to help promote strength and balance. Additionally, information on meeting Physician Quality Reporting System and National Quality Forum guidelines for falls screening (American Medical Association, 2012) was provided to help support regular, ongoing patient screening (Appendix E). Site recruitment was further assisted through existing rapport between the West Virginia University Office of Health Services Research (OHSR) and partner primary care centers. Each session lasted approximately one-hour, and was held during lunch as to avoid disruption of patient care. Lunch for all participants during the focus group sessions was also provided.

\subsubsection{Measures}

Table 2.1 provides the framework of open-ended questions guiding the focus group interviews. This framework is intended to be flexible and conversational, allowing for probing and follow-up questions as needed (Krueger, 2002). While guiding questions were provided as an outline of the structure by which the study was conducted, the focus groups retained a conversational tone with probing as needed to uncover additional information. This study aims to understand current falls screening practices in select primary care centers, the impeding and 
promoting determinants to falls screening, and the potential for EHR data-based clinical decision support to be incorporated into the care setting.

Table 2.1

Focus group discussion guide

Consent process

- Consent forms for focus group participants will be provided and completed in advance by all those agreeing to participate

Welcome and introduction

- Introductions among discussion leader, assistant, and participants

- Overview of the study purpose

- Explain to participants why they were asked to take part, and that we are having conversations like this with other primary care centers

- State that we want to learn from them, value their information, and will try to assure that everyone is heard

- Reinforce participant confidentiality and voluntary participation

- Ask permission to start audio recording to make sure we capture their thoughts and ideas

- Note that the conversation will last about 45 minutes

\section{Organizational factors}

Current culture

To get the discussion started, we'd like to know about your perspectives and experiences in caring for older patients (those 65 and older) at your clinic.

Probe as needed to learn about their experiences in caring for older patients, using this question as opportunity to hear from all participants and start a conversation flow.

Current culture

One topic we'd like to discuss in particular is accidental falls among older patients. How often do you encounter this problem? Probe as needed to determine:

- estimated extent of accidental falls among older patients;

- extent to which accidental falls are perceived as a significant issue.

Current practice

What do you think the role of primary care is in falls prevention among older patients?

Probe as needed to learn their perspectives on:

- falls risk screening and if/when screening occurs;

- what screening instrument(s) is used if any;

- patient referral processes;

- availability of community/educational resources for patients at-risk; 
Current practice

Innovativeness /

Organization norm

Additional information

Conclusion of discussion
- facilitators and barriers to screening;

- whether the approach is ad-hoc or based on policy/procedures.

There's a variety of falls-related information that can be gathered when caring for patients. What do you think about the potential for using your EHR to manage and use that data for falls screening?

Probe as needed to learn if the EHR is used, and if so the:

- documentation approaches and procedures;

- breadth of data recorded.

We've been developing a new approach to screening, using EHR data to identify patients at-risk for falls. What are your thoughts on using EHR data to identify at-risk patients?

Probe as needed using the innovation characteristics framework to help determine participants' perceptions of the:

- perceived relative advantage compared to existing procedures;

- compatibility or fit of the approach with the clinic;

- complexity or feasibility of the approach.

What other information would you like for us to know about?

- Thank the participants for talking with us.

- Offer opportunity for the participants to provide additional information at this or a later time via contact information provided.

\subsubsection{Analysis}

The focus group audio files were transcribed verbatim by a professional transcription service (Averbach Transcription, 2015). All references to identifying information, including names of persons, locations, and organizations were removed from the transcripts to ensure confidentiality in health centers, specific locations in which they serve patients, and focus group participants. The transcripts were compared to the original audio files and notes for accuracy, with edits made as appropriate. NVivo version 10.0 was used to code and categorize themes from the data (NVivo, 2015). A combined inductive approach to content analysis, allowing for patterns and themes to emerge from the data, and directed content analysis to explore areas of theory as outlined in the focus group discussion guide, was used in reviewing and coding the transcriptions (Hsieh \& Shannon, 2005; Bradley, Curry, \& Devers, 2007). Transcripts were 
independently coded and reviewed in a two-stage process by the primary and secondary

researchers until agreement on codes and themes was reached. Stage one of the coding involved development of mutually agreed-upon themes, while stage two involved a further refinement and synthesis of the data into key theory-based constructs and variables necessary for analysis. This iterative, team-based approach to coding allowed for identification of common themes, meaningful differences, patterns, and important variables (Janetti, 2005; Prine, 1998; Strauss \& Corbin, 1994). See Appendix F for the analysis codebook. Reflections on the focus group sessions were also documented by the primary researcher directly after each session to help inform the process. Initial coding schema are presented in Table 2.2.

Table 2.2

Initial focus group coding: sources and references, by focus group conversation area

Coding

1. Experiences in caring for older adults

Complex care needs

Difficulty communicating to patients

Generational differences

Informal ways of identifying falls risk

Lacking knowledge about patient

circumstances

Need for home safety

Patient independence

Patient reluctance to use assistive or safety devices

Patient transportation problems

Rewarding to care for older patients

Treating older adults differently

$\begin{array}{llr}\begin{array}{c}\text { Sources } \\ \text { (Focus groups) }\end{array} & \text { References } & \text { \% total } \\ 3 & & \\ 4 & 12 & 12.24 \\ 1 & 9 & 9.18 \\ 4 & 3 & 3.06 \\ 2 & 8 & 8.16 \\ & 3 & 3.06 \\ 2 & 5 & 5.10 \\ 4 & 29 & 29.59 \\ 3 & 14 & \\ & & 14.29 \\ 3 & 4 & 4.08 \\ 2 & 7 & 7.14 \\ 2 & 4 & 4.08 \\ & 98 & 100.00\end{array}$

3

3
43.75

56.25

100.0

3. Role of primary care is in falls prevention among older adults 
Absence of policies and procedures

Difficulty in addressing falls factors

Educating patients on falls risks

Financial barriers to falls screening

Ideas spurred on practice changes

Need for home health

Need for team-based care

Reluctance to screen

Visits not dedicated to falls prevention

$\begin{array}{ll}4 & 18 \\ 3 & 6 \\ 2 & 8 \\ 3 & 22 \\ 2 & 11 \\ 4 & 14 \\ 4 & 25 \\ 2 & 5 \\ 2 & 3\end{array}$

18

6

8

22

11

14

25

5

3

112
16.07

5.36

7.14

19.64

9.82

12.50

22.32

4.46

2.68

100.00

4. Potential for using your EHR to manage and use that data for falls screening Inconsistent use of the EHR Yes potential

$\begin{array}{ll}3 & 8 \\ 3 & 6\end{array}$

5. Thoughts on using EHR data to identify at-risk patients

Organizational barriers to EHR use 4

Technology barriers to EHR use 4

8

4

100.00

\begin{tabular}{rlr}
4 & 9 & 39.13 \\
4 & 14 & 60.87 \\
& 23 & 100.00 \\
\hline
\end{tabular}

Based on reflection and synthesis of the initial coding structure by the primary and secondary researcher, a final set of theory-based constructs and variables emerged from the data. There are four resultant variables (i.e., perceptions of the patient population, resources, team-based care, and policy and procedure) across two Organizational Change Theory-based constructs (i.e., current culture and current practice) serving as the framework for analysis (Table 2.3).

\section{Table 2.3}

Second focus group coding: sources and references, by theory-based construct

\begin{tabular}{|c|c|c|c|}
\hline Coding & $\begin{array}{c}\text { Sources } \\
\text { (Focus groups) }\end{array}$ & References & $\%$ total \\
\hline \multicolumn{4}{|l|}{ 1. Current culture } \\
\hline Perceptions of older adult patients & 4 & 55 & 33.74 \\
\hline Resources & 4 & 48 & 29.45 \\
\hline \multirow[t]{2}{*}{ Team-based care } & 4 & 60 & 36.81 \\
\hline & Total & 163 & 100.0 \\
\hline \multicolumn{4}{|l|}{ 2. Current practice } \\
\hline \multirow[t]{2}{*}{ Policy and procedure } & 4 & 68 & 100.0 \\
\hline & Total & 68 & 100.0 \\
\hline
\end{tabular}




\subsection{Results}

Focus groups were carried out in four rural, WV primary care centers. The focus groups were completed between August, 2014 and January, 2015. Participants included physicians (Medical Doctors and Doctors of Osteopathy), nurse practitioners, nurses, and medical assistants. In one instance, a health center's chief information officer took part. The number of participants per focus group range from a minimum of 6 to a maximum of 15, with an average of 10 health care team members taking part per session.

Results are organized using Stage Theory of Organizational Change constructs as a framework for identifying important variables regarding the current culture and current practice of falls screening among older adult patients in these participating primary care centers. Within the framework of current culture, results are here presented in terms of perceptions of older adult patients, resources to care for those patients, and the need for team-based care to best care for patients overall including falls screening and prevention. Within the framework of current practice, results are here presented in terms of policy and procedure in these primary care sites for a systems approach to patient and population health management.

\subsubsection{Current culture: perceptions of older adult patients}

All focus group participants were asked to share information on their perspectives and experiences in helping to care for older adults. This more general question served the dual purpose of helping participants become comfortable talking in the group setting while also allowing for information gathering on an important contextual issue on the current culture of these primary care centers.

Participants consistently reported that they find older patients to be some of the most respectful and appreciative of all age groups. Further, participants consistently expressed that the 
care they provide is worthwhile and rewarding, to the point of helping them find enjoyment in being a health care provider. As stated during Focus Group 3:

“...it's frustrating, but it's also very rewarding to take care of older people, especially those who don't have a lot of resources and ability to care for themselves, and family support, because you're really their only support they have in some situations." -Focus

\section{Group 3}

The tendency for older adult patients to want to remain independent was commonly cited. The importance placed by older patients in retaining their independence in living arrangements, in driving, and in their overall activities of daily living was consistently shared across discussions. The topic of unintentional falls naturally entered discussions of patient independence. Falls were often cited as a trigger of a larger series of events and the "biggest thing that leads to them [older adult patients] losing their independence" (Focus Group 4). Independence was cited as important enough to lead some patients to deny the presence of falls risk factors, deny use of assistive devices such as walkers, canes, and bath tub railings, and deny participation in programs to help with, for example, balance improvement. As noted in Focus Groups 1, 2, and 3:

"They evaluated my mom for a walker, told her to go ahead and get it since she had Medicaid and it helped pay for it. And she's like, 'I ain't using it. I ain't taking it." And she's like, 'I'll embarrass you.' I said, 'It'll embarrass me more if you fall on the floor than if you use that stupid thing. " -Focus Group 1

"[Patient] came in the other day, he was real unstable. I said, "You have a cane or walker? Do you need one?" Because he says he's been like that, and he's always like that. And he said, "No, I have a cane. I just didn't want to bring it." And I said, "Well, 
just seeing you walk in here, you need to use it." And I said, "I'm not saying that to be mean, but you'd much rather use your cane than fall." Because, I mean, he could barely, like, make it through the hallway. He was swaying back and forth."-Focus Group 2 “...I think that the resistance to the big leap of going from not needing any aid to be mobile and needing the simplest of aids is huge for some people, and it is the sign to many of that population that it's their first step in decline, and if they can just resist that aid, they can resist the whole decline process, which is illogical, but I think we all have some of that." -Focus Group 3

The families of the patients, on the other hand, were considered to be more accepting to suggestions regarding use of assistive devices and programs to help with physical strength and balance. From Focus Group 2:

"Yes [home health agency] did that [helping with balance]. And I don't know how effective it is or anything else. It's like a day training. But you know, they don't want that. Their families might want that for them. Yeah, I think we see that a lot more. Families wanting the home health and referrals, and fall risk assessments, and the physical therapy type things in the home. And that patients are more reluctant to agree to that, but their families want it more than they do." -Focus Group 2

Throughout the focus groups, there was a tendency for respondents to draw on stories about their own families and loved ones, as opposed to only their patients, when talking about the relationship between patient independence and falls among older adults and the perception that falls are, as a whole, underreported.

"My 70-year-old great uncle on the roof, fixin' the roof, had to be fixed, broke his hip." Focus Group 3 
"My father was in a dementia facility, He just went there in May. And he had several falls, and he fell in July and hit his head, and it killed him. And so it's a huge thing. And my mother in law died of a head injury too, from a fall in the bathroom. On the death certificate is says 'congestive heart failure.' It says 'COPD' on my mother in law's, but that's not what killed her. So I think there's a whole lot more falls that we don't know about. Those are anecdotal stories, but I think there's a whole lot of people that fall is their demise. And I don't know the answer. I wish I did." -Focus Group 1

“My mom's 62, God bless her, and I don't know, how long is her driveway? It's pretty long, and it's straight up and down, and she cleans it by herself with a shovel and a broom in winter. 'Mom, you want any help?' 'Nope, I'm getting it.' She said, 'I want to be able to do what I did when I was 40.' And I'm like, 'Mom, you're 62 years old. You're gonna' slip and break your hip one day.' 'Nope.' She said, 'Just come pick me up off the ground and I'll be all right.' I'm like, 'Okay.'-Focus Group 1

"My mom's 64, 65. She still climbs ladders and mows the hillside. Gives us all heart attacks, but she does it." -Focus Group 1

\subsubsection{Current culture: resources}

A perceived lack of resources, creating barriers to addressing falls screening and prevention among older adults was consistently expressed across focus groups. Inadequate health insurance coverage among their patient populations was a common theme. Lacking health insurance coverage is considered to be a health systems barrier to screening. As noted in Focus Group 3:

"... and I think one of the biggest challenges I have is not - and I think we all have it - is not so much dealing with them; it's more of resources, and especially in the area. 
They're all under a limited income, and getting them to and from doctor's appointments or physical therapy or what have you - even getting things ordered, because the copay, 'I can't afford that,' or 'I just got my lab bill back and it was \$300, and I can't afford that. It 'll take me a year to pay that off.' That's one of the biggest challenges that I can think, as far as taking care of them."-Focus Group 3

Reimbursement for falls screening in primary care is problematic. Lacking reimbursement tends to contribute to an overall lack of a sense of feasibility in building in-house procedures for addressing falls. For example, there was a clear tendency for focus group participants to acknowledge the importance of falls screening and the benefits of home visits to address environmental risk factors, while at the same time citing barriers as to why these issues go unaddressed. From Focus Groups 1, 3, and 4:

“That's one of the reasons why I don't think it's [falls screening] being done with such compliance. Because we don't get money. ...it's that we only do what we get reimbursed for."-Focus Group 1

“They'll let us know it they've got stuff going on at home that needs to be taken care of that they think is a problem. But you know, somebody's got to pay for that."-Focus Group 3

“...Which is the only way you can really tell what is happening in someone's home, is to have someone physically go there. And the only thing I can foresee that you could do is home visits, but out current system doesn't really allow for those very easily." -Focus

\section{Group 3}

"If we knew, what are the diagnoses that are covered, and [health care team member] had a list of 'This is what's covered, what's not.' So sometimes someone may be eligible 
for a service and we just don't know they are, or we refer them and we get turned down, but it's just because we didn't list it the right way." -Focus Group 4

Outside of the issue of care reimbursement, we find an overall sense of lacking time, personnel, and care delivery systems to address falls screening and prevention in primary care. Across the focus groups, we find a perceived lack of feasibility in incorporating standard procedures for addressing falls among older adults during routine care. As noted in Focus Groups 1, 2, and 3:

“You could automatically say, 'Anybody who's over this age, we're going to go ahead and give them fall [information].' That would be easy enough. But the problem is who's going to give them the information? Who's going to pay for them to give the information? Ten minutes on patients in the morning, that's a lot of time. Who's going to pay for that time? ...Right now you single out one or two people, and you spend that time. But if you had to do it for everyone first thing in the morning, then that would take a person a good amount of time. ....It'd be at least a full-time equivalent extra person." -Focus Group 3 "I think there's a lot that's overlooked, because we've got so many things going on." Focus Group 1

“I've got 25 things to do, I'm not going to assess your falls today." -Focus Group 1 "It depends on the patient and what all we have to do too. If somebody's just come in for a blood pressure, then it's not as much as if they're coming in for diabetes and blood pressure and cholesterol and 500 other things. " -Focus Group 1

"I get a lot of this stuff comes across from insurance companies. Medicare and Blue Cross/Blue Shield. Quality nurse comes and talks about a bunch of things, wants 
everybody screened for falls. And I have 20\% of my Alcs greater than 9. We can only deal with so many things. And I'm not going to throw not one more thing on to the providers, because they're already busy enough." -Focus Group 1

"So yes, if the patient is smoking, I like to do a smoking cessation talk every time. I like to do a cage question every time I see a drunk. I like to do intimate partner violence every time I see a person who has that there. So all these activities and little thingies, the screens, I like to do those, but if I've got 15 minutes to see a chronic, complicated patient, plus I've got to walk in there or work in there with some other problem, and plus my computer is not cooperating, that time is gone. I can't do it. I like to do the falls precautions and fall preventions and refer them to this and that, but..." -Focus Group 2

In one health center taking part in the focus groups, an in-house frail elder program was for a time instituted using grant funding. This program was used to help screen for issues in physical strength, balance, bone density, and falls risk factors among their older adult patient population. Once the grant funded ended, however, the program ceased. Participants in this focus group were particularly reflective on that program, the benefit it offered, the gap in their patient care process it left once it ended, and the sense of not being able to address these issues in the absence of the program. As noted in Focus Group 4:

"But I do think as a clinician, one of the barriers to asking about or screening for falls is that we don't have that [frail elder program] available anymore." -Focus Group 4

“...But now I know we don't have that going on, [health team member's] not doing that anymore. So if I find out that someone's falling, there's less that I can do now, because the in-home intervention is so key - the throw rugs, seeing what's in the environment. And especially if it's an elderly person coming in without family." -Focus Group 4 
"And if we don't have that grant available anymore through our health center and home health, it's like pulling teeth to get Medicaid to pay for home health services." -Focus Group 4

Finally in regard to resources, there were in some instances a sense of futility in addressing falls risk factors among older adult patients due to a general inability to effectively address those problems. While this was not the consensus across focus groups, this tone among some providers and medical care team members is a potentially important factor in the overall culture of care in these clinics. As noted in Focus Group 4:

“They don't quality for home health because their insurance won't pay for home health, they don't have the right diagnosis or whatever. It's kind of like I don't know that there's much I can do, even if I find out they're falling. So I guess a barrier for me would be there's not much I feel like I can do as a clinician currently based on resources." -Focus Group 4

“If I knew I could just quickly click 'referral to home health' and then I knew it would go through and it was covered, and it wouldn't be something that would keep coming back to me saying, 'It's not covered because they don't have this diagnosis, but what do you want to do?' this and that, then yes, I'd be more likely to screen then."-Focus Group 4 "Falls is a very complex problem. So it's also a Pandora's Box that, if you're already dealing with a lot of other medical problems, you might feel like, 'I don't have the time to try and figure out what's causing their falls. "' -Focus Group 4. 


\subsubsection{Current culture: team-based care}

Across focus groups, health care providers and team members find value in team-based care for falls screening and prevention among older adult patients. During our discussions, teambased care was described as an integrated effort of health care providers both internal and external to the primary care center. External partners included home health agencies, physical therapists, specialists, and other community-based programs. The notion of team-based care at times changed the tone of conversation from one of impossibility in addressing falls to one of possibility. This was especially true at the health center which used to have a frail elder program for risk factors screening. As noted in Focus Group 4:

"What we did - it's been a few years ago - the providers would recommend patients to me that were on high risk to fall. So we had like a criteria that we did. I'd ask them these questions and then we would have a walk test with them and all these and see how they scored. Then if they scored within a certain range, I'd go do home visits on them, and make sure, if they had throw rugs and things, recommend they get rid of those... I could have up to 50 patients I'd do home visits on three times a year. I did that for quite a while... And through grants from different people, we were able to build wheelchair ramps for people, so they didn't have to do stairs in and out of their house, and made sure they had bars, and just general safety things. So we did that for, I don't know, four or five years, maybe."-Focus Group 4

The need for established partnerships and processes to conduct regular home visits to help assess environmental risk for falls was often expressed. The desire for team-based care, however, is not acted on and remains a gap in the patient care process. 
“... Which is the only way you can really tell what is happening in someone's home, is to have someone physically go there. And the only thing I can foresee that you could do is home visits, but our current system doesn't really allow for those very easily." -Focus Group 3

“...but I find it much harder to get home health services for patients, and when it comes to falls, the first thing I'm thinking is someone to help figure out what's going on at home." -Focus Group 4

“And that's how I feel about home health services in general for vulnerable older people. Gosh, it seems like such a good way to spend health care money, to send, have a couple of visits, have a really low threshold to have a nurse go out there and evaluate someone a couple times, rather than just wait and see if they end up falling." -Focus Group 4

Moreover, there was at times admittance among focus group participants that they cannot manage falls screening and prevention on their own and that team-based care is essential to organizational movement towards addressing falls. From Focus Groups 1, 3, and 4:

"This needs to be addressed based on the risks we've identified for all of the different diseases. But how do we get around to doing that? Do have to have case managers? And now many case managers for how many problems are we going to be doing quality measures on? We'd be happy to get just one." -Focus Group 1

"If you want it [falls risk identification] to happen, somebody has to go out to the home, somebody has to be there, evaluate what the risks are at the home." -Focus Group 3

"To be honest, most of the screenings that we do are not done at a clinician level; usually it's something done at the intake by the MA. Or something that the computer just 
automatically calculates, like the BMI. The most likely I am to do a screening is if I don't have to do it personally." -Focus Group 4

Further, at times we find that focus group discussion allowed participants to ask themselves questions regarding what other health care professionals, such as home health agencies and physical therapists, could do to aid in the screening process and if these resources are available in their communities. As noted in Focus Groups 1 and 3:

"I forget which home health agency it is. I tried to get my dad to do it, where they come to your house and they have you stand on this pillow, and they do these things that are supposed to help you with your balance. Where does that come from?"-Focus Group 1 “...I guess it's possible you could ask the physical therapist to do a fall risk assessment. Because they're going to be watching them on a treadmill or stationary bike or doing any strength tests, working with them individually, will observe them for longer periods of time with activities. I don't know that they can do that; does anybody? Do PTs do fall risk assessment?"-Focus Group 3

In some instances, focus group discussion regarding team-based care and falls screening prompted commentary on delivery system design overall. As noted in Focus Groups 1 and 2: "Falls are of interest, but I think the general method of determining risk and applying that in a group effort to improved care got my interest. Because we can apply it to other situations. - Focus Group 1 "I'm going to be honest with you, until we had this [focus group] - if somebody comes in and it's obvious they're falling, I'll say, 'Oh, gosh, let's write you for a quad cane, whatever.' That's pretty much as far as it goes, I haven't referred a lot for gait training, 
to PT or anything. And if I do, are that gonna go? I should do the referrals, and at least I've done everything I can, but..."-Focus Group 2

"Care coordinators. That may be something we can incorporate, because my vision for these people - and I've talked about this for years - is to be able to look at the provider's schedule next week, review those patient charts... The preventive care and fall risk assessment may be something we can incorporate into that review of the chart and take it to the nurse at the beginning of the week and say, 'These are the patients that are coming in this week. These are the gaps we need to close in their care.' -Focus Group 2

\subsubsection{Current practice: policy and procedure}

Across all focus groups, we find a consistent lack of policy and procedure for addressing falls among older adult patients. Variations in care span the patient care process, from the ways in which risk factors are identified, to the ways in which data are entered into the EHRs, to the ways in which providers care for their patients. From Focus Group 2:

"I don't think there's a formal screening I use per se, but I watch them as they get up from the chair and walk over to the exam room. I usually watch them walking down the hallway, if they're ambulating at al. And of course just questions."

"Yeah, because if I see you come in and you do what I call the furniture walk... 'What do Ineed to grab on my way to get there?' I'll grab this chair. Ooh, it's a rolling chair." “Absolutely, if you're holding onto a chair, we know you're at risk. Or if we see you totter. Yeah, you're right." -Focus Group 2

In our discussions, providers and care team members were at times candid about the absence of policies and procedures, contrasting falls care with that of diabetes, obesity, and tobacco 
cessation - all of which involve commonly collected metrics often used for quality of care improvement and are also for required reporting to agencies such as the Health and Resources Services Administration. From Focus Groups 2 and 4:

"I don't think it [falls risk screening] happens all the time. Like automatically checking an Alc in a diabetic. I don't think it's a reflex, 'We need to do it,' type of thing yet. I don't think that the awareness is all that great yet, and I don't think we comply with doing that every single visit also." - Focus Group 2

"I don't think we have a check option like we do in... where BMI has been counseled and documented, tobacco cessation has been counseled and documented. We don't have a spot there to say fall risk assessed and documented."-Focus Group 2

“We don't know about them. We don't ask. I mean, I don't ask. That's not one of the things we ask, 'Do you fall a lot?'”-Focus Group 2

"Especially when their blood pressure's normal on presentation. If it's low, maybe they would have an inclination to do it on prompt, but I think if the blood pressure's normal, nobody would have a tendency to do all the stats." -Focus Group 2

"I don't screen unless it's obvious..." -Focus Group 2

"But is there something more that we should be doing? Like should more than eight meds on their list trigger an in-depth, maybe polypharmacy? Should something else trigger it? Or should we just leave each individual clinician to use their magical medical powers." -Focus Group 4

A conversation between providers and nurses during Focus Group 4 regarding entry of falls history data into the EHR reveals not only a lack of policy and procedure but moreover the need 
for training on and consensus in best practices for using these systems for patient care. From Focus Group 4:

Participant A: "You put it [history offalls] under the diagnosis, don't you?"

Participant B: "Yeah, I put it on the diagnosis. I put it on the problem list if someone had frequent or recurrent falls - I'd put it on the problem list because I think that's really important to keep that there as one of their medical diagnoses."

Participant C: "But just one fall, I wouldn't put it on the problem list, and I would be willing to bet that the EHR probably does have some checkbox for falls..."

Participant A: "I use different parts of the chart more so than they [nurses] do, but I've never seen any, unless you just want to put it in a dropdown box or something."

Participant B: "I put it on the list to investigate, I don't think we have a good way of documenting -." -Focus Group 4

Similar discussion occurred in Focus Group 2, this time with thought given to the impact data entry habits can have on continuity of patient care. From Focus Group 2:

"I bet there's something in the EHR, but the problem is there's so many things to check off and ask in the intake that you can get... And a lot of it isn't charted. The other people see it, though. And what you might think is irrelevant might not be irrelevant to somebody else. So if you didn't chart it, then somebody else didn't see it, and they think, 'Well, that person's okay, 'so it depends on what you think is relevant versus their information as well." -Focus Group 2

Further, we find a lack of knowledge about the clinical data relating to falls being routinely collected and how it can be used for falls screening. This was especially apparent in Focus Group 2 in which participants were unable to identify key metrics relating to falls risk identification: 
"And I'm thinking, what do we have in there right now that might trigger this flag?

Nothing. Unless the blood pressure's real high." -Focus Group 2

At times, we found that discussion of the EHRs helped to give context to the lack of

policy and procedure in these health centers - for falls among older adults as well as other health conditions. We find the tendency for the clinical decision support afforded in the EHRs to serve as a proxy for policies and procedures. However, while clinical decision support stands to be helpful in promoting national care guidelines and overall quality of care improvement, these tools are often cited as nuisances and used after the fact rather than at point-of-care as intended. From Focus Group 2:

Participant A: "I'm sure they're [decision support] useful, but they're a pain in the butt. [laughter] They are a pain, because half the time... I don't ask her if she had a Pap last year. That's the kind of thing."

Participant B: "You are supposed to ask them that."

Participant A: “You know what I'm saying. They've already left by the time I look at it. Well, I rely on it after the fact, and then I send [health care team member] the flag and say, 'Call her and ask her if she's had a Pap in the last year,' which is terrible. But I mean, I'm sure they're a good thing. I'm sure they're a wonderful thing."

\subsection{Discussion}

Focus group discussions reveal, overall, that these primary care sites are under a tremendous amount of pressure to meet the needs of their patients. The collective narrative from these focus groups reveals a caring, dedicated collection of health care providers and team members helping to care for older adult patients with complex health care needs and wanting to remain independent. While falls risk identification and prevention are acknowledged as 
important, the health care providers and team members are at a deficit for resources to adequately address the complex care needs of older adults within the time and energy constraints of brief office visits. While the need for team-based care within the clinic and linkages with resources and expertise outside of the clinic is acknowledged, we find a consistent sense of frustration and futility in building and sustaining such a system - especially as it relates to reimbursement. Further, we find an overall lack of readiness to appropriately use health information technology and inability to systematically document and use EHR data to inform falls screening and prevention. Moreover, we found a lack of awareness on the part of providers that data germane to falls risk identification are routinely collected in EHRs. This stands in contrast to metrics for chronic health conditions which are more commonly tracked and expected to be available for required reporting needs. Lastly, the dearth of not only policies and procedures to address falls screening and prevention but moreover the lack of readiness to acknowledge the problem as capable of being addressed in primary care is a central issue. Decision support offered by the EHRs, which is itself often bypassed, tends to be a proxy for actual policies and procedures. The EHRs are viewed much more as a patient-level tool for recording information rather than a population-level tool capable of providing data necessary for efforts in prevention, identification of at-risk patient populations, and population health management. Overall, we find the issues raised during focus group discussions to be informative of not only falls screening and prevention but to overall efforts in systems improvement through a closer understanding of contextual issues in providing care in rural West Virginia. These findings can help inform not only public health efforts in falls prevention but efforts to effectively partner with primary care on quality of care improvement and systems change efforts. 


\subsubsection{Limitations}

This study is based on a non-randomized sampling of WV primary care centers, and is therefore limited in terms of its generalizability. Further, the focus group interviews are susceptible to facilitator bias which can harm the validity and reliability of the study findings (Miles \& Huberman, 1994). Focus groups are also subject to both positive and negative group effects; ranging from problems due to dominant group members, unwillingness to share in a group setting, and issues in power and position (Kaplowitz \& Hoehn, 2001). However, this observational study can facilitate understanding of current falls screening practices in select primary care centers, the impeding and promoting determinants to screening, and the potential for EHR data-based clinical decision support to be incorporated into the care setting. Understanding these key issues is prerequisite for increasing screening among older adults.

\subsection{Conclusions}

Focus group discussion with primary care partners was revealing in terms of helping to understand falls risk screening practices in these sites, the impeding and promoting determinants to screening, and the potential for EHR data-based clinical decision support to be incorporated into the care setting. Basing our findings in the context of Stage Theory of Organizational Change, we find a significant lack of readiness to innovatively use routinely collected EHR data for population health management for falls prevention due to a myriad of environmental barriers and perceived obstacles to change. The topic of falls risk identification and prevention is a rarely discussed topic across these primary care centers. Likewise, the extent to which older adult patients are screened for risk and referred for services is sparse at best. While national care guidelines call for the gold-standard in falls risk identification (the Timed Get Up and Go test), the four primary care centers in this study use no form of consistent, standardized screening. 
Data routinely collected in EHRs such as age, demographics, diagnoses, and medications, while applicable to falls risk identification, are not viewed as such. Moreover, key falls data necessary to accurately identifying important population health metrics, such as history of prior falls, is not collected in standardized, well-understood ways. Our focus group discussions spurred renewed and at times initial discussion on the importance of falls among older adults and the ability for this issue to be addressed in the primary care setting. Public health partnerships to support primary care through well-informed, sensitive efforts in practice facilitation may help overcome some of the environmental and organizational barriers identified in this study. 
Chapter 3 


\section{Chapter 3}

\section{Developing methods in repurposing electronic health record data for identification of older adults at-risk for unintentional falls}

\subsection{Introduction}

Detecting community-dwelling, older adults at-risk for falling poses a serious challenge. The Timed Get-Up-and-Go test is the gold-standard assessment recommended by the USPSTF for determining falls risk (Moyer, 2012). However, this test is best considered within a larger battery of assessments to more definitively measure physical function (Lindsay, James, \& Kippen, 2004; Piva, Fitzgerald, Irrgang, Bouzubar, \& Starz, 2004) and is dependent upon clinicians using standard procedures and equipment (Siggeirsdóttir, Akranes, Jónsson Jr., \& Iwarsson, 2002). The test can be completed in less than a minute, but this additional task can be challenging to incorporate into brief office visits given the complex health needs of older patients (Boyd, et al., 2005). Nationally, screening for falls risk is completed only 30-37\% of the time (Hayden, et al, 2004).

Given the need for efficient, systematic primary care screening for falls risk, exploring use of EHR data to identify at-risk patients is warranted. EHRs have the potential to be valuable tools for health outcomes research in primary care (Dean, et al., 2009; Ethredge, 2010; Hanna, Anderson, \& Maddox, 2005; Weiner, Lyman, Murphy, \& Weiner, 2007; de Lusigna \& van Weel, 2005) and a critical component in reducing preventable deaths through increased adherence to preventive services (Farley, Dalal, Mostashari, \& Frieden, 2010). However, EHRs are primarily designed to support patient-level care and often lack population-level reporting and health analytics features essential to public health efforts (Benin, et al., 2005; Dean, et al., 2009; Kukafka, et al., 2007; Terry, et al., 2010; Tolar \& Balka, 2011). Moving the EHR data to an 
external system allows for more in-depth querying of the data, data transparency in that key data within the EHR (i.e., patient diagnoses, demographics, vitals, laboratory results, and services) can be queried for coding consistency and completeness, and analysis of free text or narrative data. Analysis of free text or narrative data is of particular interest due to the potential for essential information to be found in these locations and not in the coded areas of the EHR data (Chen, Hripcsak, \& Friedman, 2006; Friedman \& Hripcsak, 1999; Gerbier, et al., 2011; Ware, Mullett, \& Jagannathan, 2009; Botsis, Hartvigsen, Chen, \& Weng, 2010; Hayrinen, Saranto, \& Nykanen, 2008).

While repurposing EHR data for the identification of patients at-risk for some chronic health conditions has been explored (Baus, Wood, Pollard, Summerfield, \& White, 2013; Hanna, Anderson, \& Maddox, 2005; Terry, et al, 2010), to date there have been no published studies on using EHR data for identification of older adults at-risk for falls. There is an absence of methodology and guidelines for performing a search for this specific population. Given this gap in knowledge, this study examines the utility of importing EHR data into an external clinical information system to systematically identify older patients at risk for falls, incorporating methods for determining the accuracy and completeness of the data, or internal validity. Considering the tendency for important information to be entered into EHRs through free-text or narrative as opposed to data entry (Chen, Hripcsak, \& Friedman, 2006; Friedman \& Hripcsak, 1999; Gerbier, et al., 2011; Ware, Mullett, \& Jagannathan, 2009; Botsis, Hartvigsen, Chen, \& Weng, 2010; Hayrinen, Saranto, \& Nykanen, 2008), a sub-aim of this study uses natural language processing methods to assess the potential for and value of additional falls risk information from free text or narrative data in the EHR. 
This study explores the feasibility of using de-identified EHR data to identify cases of older patients at-risk for falls among select WV primary care centers. The research question is whether use of an external clinical information system to analyze EHR data is a viable option to gather data pertinent to the identification of at-risk patients in that key risk criteria can be gathered from existing data, assembled for analysis, and examined for internal validity. The outcome of interest is development of methods in repurposing EHR data to identify this particular at-risk patient population for the purpose of early identification of falls risk and efforts toward prevention.

\subsection{Methods}

This nonexperimental retrospective study examines the utility of importing EHR data into an external clinical information system to systematically identify older patients at risk for falls. Previous research points to the common limitations of EHRs in not having functionality necessary for analysis and research, as they are instead designed primarily to support patient care (Benin, et al., 2005; Dean, et al., 2009; Kukafka, et al., 2007; Terry, et al., 2010; Tolar \& Balka, 2011). Given this limitation, this research repurposes EHR data for falls risk identification, paying particular attention to determining the value added in data gathered from various areas of the medical record including free text notes. This expanded use of EHR data increases opportunity to transform data collected at the time of patient care into knowledge that can be applied to better target services and intervention to patients in need, inform health care decisions, and bolster practice-based research (Okun, et al., 2013). Further, this approach offers the advantage of moving from an acute model of patient-by-patient screening to one of a planned, population model of data-driven clinical decision support for falls risk identification. 
Data were gathered using extract, transform, and load (ETL) methodology (Business Intelligence Insider, 2014). The ETL process here used involves extracting data from the EHR, being the origin of the data, transforming those data into a format capable of analysis, and then loading those data into a common repository for verification and analysis.

The extract process was completed via secure desktop connections between OHSR and the participating primary care centers. Appropriate data were selected and collected for analytical processing using SAP Business Objects (SAP Business Objects, 2013). This is proprietary software linked to the EHR. SAP Business Objects provides a mechanism for exporting data from the EHR to .DAT format, which is a text file format. This software is intended for use by primary care center administration, technical support staff, and quality improvement partners to generate reports and export data from the EHR.

Transformation of the .DAT files occurred using a Microsoft Access-based clinical information system (West Virginia University Office of Health Services Research, 2013). This tool is open-source, public domain software shown effective in previous research analyzing EHR data for diagnostic coding (Baus, Hendryx, \& Pollard, 2012) and in identifying patients at-risk for diabetes (Baus, Wood, Pollard, Summerfield, \& White, 2013). At this stage, data were deidentified, transformed into variables specific to falls risk identification, and prepared for more in-depth analysis. Data were de-identified using the Safe Harbor Method of data de-identification (US Department of Health \& Human Services, 2014). Data excluded from the resultant deidentified data set are: patient names; zip codes; all elements of dates (expect for year) including dates of birth; all ages over 89 and all elements of dates including year indicative of such age; all contact information; social security numbers; medical record numbers; health plan beneficiary numbers; and all other unique identifiers. Given the Safe Harbor Method of de-identification, 
indications of dates were transformed into time intervals in days relative to each patient's first date of visit or service. This allows for determination of duration and sequence in data elements while preserving the de-identification standard. Data transformation steps occurred via secure desktop connections, allowing only de-identified data to be shared with OHSR.

De-identified data were loaded from the Microsoft Access-based clinical information system into JMP Pro version 11.0, serving as the common data repository for analysis. EHR data completeness and accuracy, measures of internal validity, were examined using JMP by calculation of percentages of missing, out-of-range, and questionable results for each data element (Chan, Fowles, \& Weiner, 2010). As an added check, cases with and without expected medications by diagnosis were calculated under the premise that a larger proportion of unmatched cases would indicate unmatched medications and the need to reexamine data import specifications.

Natural language processing techniques were used to examine potential for value-added information from free text or narrative data in the medical record. This was an iterative process, examining case finding ability through a successive series of search term refining. Using string handling functions in Microsoft Access Visual Basic for Applications (VBA), pertinent clinical narrative for falls risk were identified, extracted, and coded into the same database format as the coded EHR data to retain continuity in database structure to help ensure the information could be presented in a way which is suitable for use by clinicians and researchers (Chen, Hripcsak, \& Friedman, 2006). Value added in locating data throughout various parts of the medical record (i.e., structured, semi-structured, and free text) was determined through examination of percent of cases missed when accounting for International Classification of Diseases $9^{\text {th }}$ Revision (ICD9) or Current Procedural Terminology (CPT) coding alone. 


\subsubsection{Participants}

This study is a nonexperimental retrospective analysis of de-identified EHR data from two primary care center organizations, representing nine physical locations excluding schoolbased health centers and dental clinics, partnering with OHSR. These centers are part of a larger network of primary care centers in the state strategically positioned in medically underserved areas (Kaiser Family Foundation, 2013; Rural Assistance Center, 2013).

Purposive sampling is used to identify primary care organizations for inclusion. Inclusion criteria are: 1) established partnership and de-identified EHR data sharing with OHSR; and 2) use of an EHR which allows for export of the necessary data. De-identified data sharing from these centers to OHSR is made possible through signed business associate agreements and memoranda of understanding. This study was classified as non-human subjects research by the WVU Office of Research Integrity and Compliance (protocol number 1402217616) as it involves secondary data that do not include Health Insurance Portability and Accountability Act protected information (Appendix G).

\subsubsection{Measures}

Three categories of modifiable risk factors are associated with falls among older adults: biological, behavioral, and environmental (Stevens \& Schuster, 2013). The primary risk criteria for falls included in this study are biological and behavioral in nature (i.e., intrinsic) as these data elements are more apt to be gleaned from EHR data. Criteria used to identify falls risk reflect current falls prevention guidelines presented in a systematic review from current USPSTF guidelines and meta-analysis on falls risk factors among community-dwelling older adults (Deandrea, et al., 2010; Michael, et al, 2010). Key variables of interest are: being greater than or equal to 65 years of age; being female; gait or balance impairment; having a history of falls; fear 
of falling; vision impairment; hearing impairment; diagnosis of Parkinson's disease;

dizziness/vertigo; cognitive impairment; use of a walking aid or device; current prescription for a sedative medication; current prescription for an anti-epileptic medication; current prescription for an antihypertensive medication; and currently taking four or more medications, also known as polypharmacy. These variables coincide with data elements commonly collected in EHRs. Pertinent clinical findings regarding falls assessment and risk factors were extracted from the general notes portion of the encounter data. Appendix $\mathrm{H}$ lists the priority factors or variables, indications of the three potential locations in which the data were located (i.e. structured or coded data, semi-structured Medcin findings, and/or free text information), and the coding used to locate the data in each location of the EHR data.

This core set of variables was expanded to include a set of secondary variables, based on a literature review of potential falls risk factors, with the intent to examine the association among a more extensive set of variables and unintentional falls. Expanded factors or variables of interest are: race; ethnicity; insurance status; falls assessment; falls guidance; hypertension; hypotension; dementia; osteoporosis; muscle weakness; rheumatoid arthritis; type I diabetes; type II diabetes; diabetic retinopathy; diabetic neuropathy; epilepsy; current prescription for rheumatoid arthritis medication; current prescription for vertigo medication; current prescription for dementia medication; current prescription for type I diabetes medication; current prescription for type II diabetes medication; current prescription for anti-epileptic medication; current prescription for hypotension medication; current prescription for osteoporosis medication; current prescription for Parkinson's disease medication; height; weight; body mass index (BMI); systolic blood pressure; and diastolic blood pressure. The prescription variables were included on this list for internal validity purposes, and insurance status was included to account for potential 
differences among patient groups. Appendix I lists the expanded set of variables, indications of the three potential locations in which the data were located (i.e. structured or coded data, semistructured Medcin findings, and/or free text information), and the coding used to locate the data in each location of the EHR data. Appendix $\mathbf{J}$ lists falls risk factors with reference to peer reviewed literature citing those factors and an indication of the risk category (i.e., biologic and/or behavioral).

\subsubsection{Analysis}

Data for this study are analyzed in-part using a Microsoft Access-based patient clinical information system. This is an intentional choice to facilitate the later development of clinical decision support tools which can be shared with partner centers for their use in quality of care improvement efforts. Queries were built in the system to search the EHR data to: 1) identify unduplicated, active patients age 65 and older and account for demographic characteristics (i.e., gender, race, ethnicity, and health insurance information); 2) identify from that subset patients with a current diagnosis of a cognitive impairment, dizziness/vertigo, a history of falls, gait/balance impairment, Parkinson's disease, vision impairment, or other secondary, targeted health conditions; 3) identify from the subset in step 1 patients with active prescriptions for an anti-anxiety, anti-depressant, anti-epileptic, anti-hypertensive, anti-psychotic, phenothiazine, or sedative medication; 4) identify patients with current prescriptions for four or more medications, known as polypharmacy; and 5) identify patients based on the composite of demographic, diagnostic, and medication risk factors. Microsoft Access VBA modules were developed to handle the free text or string functions to provide a natural language processing framework to assess the potential for and value of additional falls risk information from free text or narrative data in the EHR. 
Demographic characteristics of the patient populations were performed by univariate analysis on the EHR data using JMP Pro version 11.0. Data completeness and accuracy was examined through calculation of percentages of missing, out-of-range, and questionable results for each data element (Chan, Fowles, \& Weiner, 2010). Value added in locating data throughout various parts of the medical record (i.e., structured, semi-structured, and free text) was determined through examination of percent of cases missed when accounting for ICD-9 and CPT coding alone.

\subsection{Results}

The datasets from the two primary care organizations included in this study are comprised of nine unique locations excluding school-based health centers and dental clinics. Among these nine sites, there were 50,433 unique patients. Of these, 43,531 patients (86.3\%) were determined to be active based on having at least one documented office visit, service, or laboratory test within 3 years of the date of data extraction (3/31/2014). Among the 43,531 active patients, 3,933 (9.03\%) were age 65 and older (Table 3.1). This finding is slightly lower than recent Uniform Data System results from state-wide FQHCs for patients 65 and older for 2013 which is $12.8 \%$, yet more comparable to national $\mathrm{FQHCs}$ at $7.0 \%$ patient population age 65 and older. (US Department of Health and Human Services - Health Resources and Services Administration, 2013).

Table 3.1

Target patient population (patients age 65 and older)

Total unduplicated patients

\begin{tabular}{ll}
\multicolumn{1}{c}{ Number } & \multicolumn{1}{c}{ Percent } \\
\hline 50,433 & -- \\
43,531 & 86.3 \\
3,933 & 9.03 \\
\hline
\end{tabular}


Table 3.2 provides demographic data for the 3,933 patients age 65 and older. While statewide data for $\mathrm{FQHC}$ patients were sought for comparability, only gender statistics are available for the 65 and older patient population (87.9\% female; 12.1\% male) (US Department of Health and Human Services - Health Resources and Services Administration, 2013). Demographics in Table 3.2 detail patient age categories, gender, race, ethnicity, and health insurance information. Patients tend to be: age 65-74 (62.1\%) with a mean age of 73.5 years; female (61.3\%); White (95.7\%); Not Hispanic/Latino (99.1\%); with Medicare as a source of insurance (63.1\%). Data completeness and quality were strong as: all demographic data were coded consistently, likely attributable to standardization in the EHR data selections upon data entry; there were no missing data (i.e., empty cells) across these metrics; only $0.1 \%$ refused to report race or having race marked as unreported; and only $0.2 \%$ refused to report ethnicity or having ethnicity marked as unreported. 
Table 3.2

Demographic data for patients age 65 and older

\begin{tabular}{|c|c|c|c|}
\hline \multicolumn{2}{|c|}{ Active pateints age 65 and older } & Number & \multirow[t]{2}{*}{ Percent } \\
\hline \multirow{4}{*}{ Age } & & 3,933 & \\
\hline & $65-74$ & 2,443 & 62.1 \\
\hline & $75-84$ & 1,069 & 27.2 \\
\hline & 85 and older & 421 & 10.7 \\
\hline \multirow{2}{*}{ Gender } & Female & 2,411 & 61.3 \\
\hline & Male & 1,522 & 38.7 \\
\hline \multirow{7}{*}{ Race } & American Indian or Alaska Native & 1 & 0.0 \\
\hline & Asian & 7 & 0.2 \\
\hline & Black or African American & 148 & 3.8 \\
\hline & Multiple races & 7 & 0.2 \\
\hline & Other Pacific Islander & 1 & 0.0 \\
\hline & Unreported/Refused to report & 4 & 0.1 \\
\hline & White & 3,765 & 95.7 \\
\hline \multirow{3}{*}{ Ethnicity } & Hispanic/Latino & 27 & 0.7 \\
\hline & Not Hispanic/Latino & 3,899 & 99.1 \\
\hline & Unreported/Refused to report & 7 & 0.2 \\
\hline \multirow{4}{*}{$\begin{array}{l}\text { Insurance } \\
\text { source }\end{array}$} & Medicaid & 268 & 6.8 \\
\hline & Medicare & 2482 & 63.1 \\
\hline & Private & 1178 & 30.0 \\
\hline & Public & 5 & 0.1 \\
\hline
\end{tabular}

Table 3.3 provides vitals data for the 3,933 patients age 65 and older. Vitals detail patient height, weight, BMI, and systolic and diastolic blood pressures. In general, patients tend to be overweight with relatively controlled blood pressure. However, a chi-square test of independence was performed to further examine the relation between age and BMI. The relation between these variables was significant, $\chi^{2}(1, N=3607)=127.3, p<.0001$. Patients age $65-84$ were more likely to be overweight or obese than patients 85 and older. A check on data completeness and quality reveal some issues, with $8.0 \%$ of patients 65 and older having no documented height in their medical records, $2.9 \%$ having no documented weight, and $1.3 \%$ having no documentation of systolic or diastolic blood pressure readings. Interestingly, the majority of patients with these 
data missing are in the 65-84 age range (90.1\% height, $91.1 \%$ weight, $91.1 \%$ BMI, $89.3 \%$ systolic, $89.8 \%$ diastolic).

Table 3.3

Vitals data for patients age 65 and older

\begin{tabular}{lllllll}
\hline & $\mathrm{N}$ & $\%$ missing & Mean & Minimum & Maximum & $\begin{array}{c}\text { Std } \\
\text { Deviation }\end{array}$ \\
\cline { 2 - 7 } & 3620 & 8.0 & 65.4 & 50 & 79 & 3.9 \\
Height (in) & 3818 & 2.9 & 178.64 & 64.6 & 417.0 & 42.9 \\
$\begin{array}{l}\text { Weight (lbs) } \\
\text { Body mass index }\end{array}$ & 3607 & 8.3 & 29.3 & 13.8 & 60.4 & 6.4 \\
$\begin{array}{l}\text { Systolic blood pressure } \\
\text { (mmHg) }\end{array}$ & 3883 & 1.3 & 130.2 & 72 & 394 & 17.9 \\
$\begin{array}{l}\text { Diastolic blood pressure } \\
\text { (mmHg) }\end{array}$ & 3883 & 1.3 & 73.9 & 28 & 238 & 10.6 \\
\hline
\end{tabular}

Priority health conditions relating to unintentional falls were identified in a step-wise process using data from multiple areas of the EHR in order to build a data set as complete as possible. These areas are: 1) ICD-9 coding; 2) Medcin findings which are semi-structured data; 3) free text notes; and 4) vitals as they relate to both high and low blood pressure diagnoses. Table 3.4 provides data on: ability to identify patients by condition based on use of ICD-9 codes alone; cases identified with the addition of Medcin findings; cases identified with the addition of free text searches; cases identified with the addition of blood pressure results for hypertension and hypotension specifically; total unduplicated counts for each condition; percent of patients missed by ICD-9 coding alone; and indication of prevalence of each condition among patients 65 and older. ICD-9 coding alone missed from a minimum of $1.2 \%$ of cases (diabetes type 2 ) to a maximum of $98.1 \%$ of cases (vision impairment), with a median of $39.8 \%$ of cases missed across all conditions. Looking to multiple areas of the EHR data to identify patients with priority health conditions offers a clear advantage in case finding. Noteworthy, fear of falling, which is one of 
the priority falls risk metrics, was identified in only $1(0.02 \%)$ patient record across all search methods. Likewise, use of a walking aid was identified in only $6(0.1 \%)$ patient records. Those instances were identified using free text notes as opposed to coded information. Appendix $\mathrm{H}$ provides information on the specific text string used to search for this key word.

Table 3.4

Counts of patients by diagnoses according to search criteria

\begin{tabular}{|c|c|c|c|c|c|c|c|c|c|c|}
\hline \multirow[b]{2}{*}{ Condition } & \multirow{2}{*}{$\begin{array}{c}\text { ICD-9 } \\
\text { coding }\end{array}$} & \multicolumn{2}{|c|}{$\begin{array}{l}\text { Medcin } \\
\text { finding }\end{array}$} & \multicolumn{2}{|c|}{ Notes } & \multicolumn{2}{|c|}{ Vitals } & \multirow{2}{*}{$\begin{array}{l}\text { Undup } \\
\text { Count }\end{array}$} & \multirow{2}{*}{$\begin{array}{l}\% \text { missed } \\
\text { ICD-9 } \\
\text { coding } \\
\text { alone }\end{array}$} & \multirow{2}{*}{$\begin{array}{c}\% \\
65+\end{array}$} \\
\hline & & Total & Added & Total & Added & Total & Added & & & \\
\hline Arthritis & 84 & 99 & 23 & 0 & 0 & . & . & 107 & 21.5 & 2.7 \\
\hline $\begin{array}{l}\text { Cognitive } \\
\text { Impairment }\end{array}$ & 63 & 65 & 44 & 3 & 3 & . & . & 110 & 42.7 & 2.8 \\
\hline Dementia & 142 & 162 & 28 & 5 & 0 & . & . & 170 & 16.5 & 4.3 \\
\hline $\begin{array}{l}\text { Diabetes } \\
\text { type } 1\end{array}$ & 106 & 110 & 21 & 0 & 0 & . & . & 127 & 16.5 & 3.2 \\
\hline $\begin{array}{l}\text { Diabetes } \\
\text { type } 2\end{array}$ & 1178 & 533 & 12 & 11 & 2 & . & . & 1192 & 1.2 & 30.3 \\
\hline $\begin{array}{l}\text { Diabetic } \\
\text { Neuropathy }\end{array}$ & 41 & 97 & 64 & 2 & 1 & . & . & 106 & 61.3 & 2.7 \\
\hline $\begin{array}{l}\text { Diabetic } \\
\text { Retino- } \\
\text { pathy }\end{array}$ & 43 & 61 & 22 & 2 & 2 & . & . & 67 & 35.8 & 1.7 \\
\hline $\begin{array}{l}\text { Dizziness / } \\
\text { Vertigo }\end{array}$ & 575 & 0 & 0 & 67 & 39 & . & . & 614 & 6.4 & 15.6 \\
\hline Epilepsy & 48 & 71 & 41 & 0 & 0 & . & . & 89 & 46.1 & 2.3 \\
\hline $\begin{array}{l}\text { Essential } \\
\text { Hyper- } \\
\text { tension }\end{array}$ & 2400 & 2574 & 340 & 38 & 6 & 224 & 27 & 2773 & 12.2 & 70.5 \\
\hline $\begin{array}{l}\text { Fear of } \\
\text { Falling }\end{array}$ & 0 & 0 & 0 & 1 & 1 & . & . & 1 & -- & 0.02 \\
\hline $\begin{array}{l}\text { Gait / } \\
\text { Balance } \\
\text { Impairment }\end{array}$ & 106 & 149 & 94 & 7 & 4 & . & . & 204 & 48.0 & 5.2 \\
\hline
\end{tabular}




\begin{tabular}{lcccccccccc}
$\begin{array}{l}\text { Hearing } \\
\text { Impairment }\end{array}$ & 214 & 457 & 296 & 9 & 4 &. &. & 514 & 58.4 & 13.1 \\
$\begin{array}{l}\text { History of } \\
\text { Falls }\end{array}$ & 51 & 72 & 37 & 47 & 45 &. &. & 133 & 61.7 & 3.4 \\
$\begin{array}{l}\text { Hypo- } \\
\text { tension }\end{array}$ & 117 & 102 & 2 & 4 & 0 & 74 & 66 & 185 & 36.8 & 4.7 \\
$\begin{array}{l}\text { Muscle } \\
\text { Weakness }\end{array}$ & 90 & 261 & 135 & 2 & 2 &. &. & 227 & 60.4 & 5.8 \\
$\begin{array}{l}\text { Osteo- } \\
\text { porosis }\end{array}$ & 466 & 465 & 259 & 27 & 9 &. &. & 734 & 36.5 & 18.7 \\
$\begin{array}{l}\text { Parkinson's } \\
\text { Disease }\end{array}$ & 23 & 54 & 31 & 1 & 0 &. &. & 54 & 57.4 & 1.4 \\
$\begin{array}{l}\text { Vision } \\
\text { Impairment }\end{array}$ & 9 & 460 & 455 & 10 & 6 &. &. & 470 & 98.1 & 12.0 \\
\hline
\end{tabular}

In sum, there were 238 instances in which falls were documented among patients 65 and older. These falls were documented across 133 unique patients. Falls range from a minimum of one documented fall among 80 patients $(60.1 \%)$ to a maximum of 16 documented falls among one patient $(0.7 \%)$, with a median of one documented fall.

Free text information was especially important in the identification of patients with a history of falls, with $33.8 \%$ of all cases added through free text notes. Even with this expanded search method however, only 133 patients (3.4\%) have an indication in their medical records of having had an unintentional fall at some point in the past. This is likely a low estimate, as one out of three adults aged 65 and older falls each year nationwide, yet less than half of these individuals talk with their healthcare providers about falling (Centers for Disease Control and Prevention, 2014). Free text searches were also developed to identify falls cases using the derivations "slip," "trip," and "stumble." Only 1 patient record $(0.02 \%)$ had an indication of having had stumbled. This notation, however, included no mention of a fall and therefore affords no value added to case finding. No patient records were identified through "slip" or "trip." Appendix I provides information on the specific text strings used to search for these key words. 
A recent systematic review from current USPSTF guidelines and meta-analysis on falls risk factors among community-dwelling older adults (Deandrea, et al., 2010; Michael, et al., 2010) highlights sedatives, anti-epileptic medications, and antihypertensive medications as associated with increased risk for unintentional falls. Further, polypharmacy, defined as currently taking four or more medications (Deandrea, et al., 2010; Michael, et al., 2010), is also highlighted as associated with increased risk for unintentional falls. Table 3.5 provides information on counts of patients identified as having current prescriptions for these priority medications and polypharmacy among active patients 65 and older. Data on medications were found in the medications portion of the EHR data only. Eighty-five percent of patients 65 and older are characterized with polypharmacy.

\section{Table 3.5}

Counts of patients with select medications and polypharmacy

\begin{tabular}{|c|c|c|c|}
\hline Medication category & Count & $\%$ Patients & $65+$ \\
\hline Anti-epileptic & 597 & & 15.2 \\
\hline Anti-hypertensive & 1,750 & & 44.5 \\
\hline Sedative & 294 & & 7.5 \\
\hline Polypharmacy & 3343 & & 85.0 \\
\hline
\end{tabular}

As an added check on data quality, percent of patients by health conditions with active prescriptions for appropriate medications were calculated (Table 3.6). The EHR data exports offer medication information through the brand and generic names of the medications, as opposed to coding schema, which decreases the ability to match. Appendix I provides detailed information on the brand and generic medications used to create these classes of medications. Results range from a minimum of $0.0 \%$ of patients with hypotension with record of a current prescription for that condition to a maximum of $70.4 \%$ of patients with Parkinson's disease with 
record of a current prescription for that condition, with a median of $45.4 \%$ of patients with

current prescriptions for appropriate medications across all conditions.

Table 3.6

Counts of patients with select health conditions and current prescription for appropriate medications

\begin{tabular}{crr}
\hline \multicolumn{1}{c}{ Condition } & Number & Percent \\
\hline Total active patients 65 and older & 3933 & -- \\
Active patients 65 and older with dementia & 170 & 4.3 \\
$\bullet \quad$ with current prescription for dementia & 4 & 2.3 \\
Active patients 65 and older with diabetes type 1 & 128 & 3.2 \\
$\bullet \quad$ with current prescription for diabetes type 1 & 68 & 53.1 \\
Active patients 65 and older with diabetes type 2 & 1192 & 30.3 \\
$\bullet \quad$ with current prescription for diabetes type 2 & 827 & 69.4 \\
Active patients 65 and older with dizziness/vertigo & 614 & 15.6 \\
$\bullet \quad$ with current prescription for vertigo & 136 & 22.1 \\
Active patients 65 and older with epilepsy & 89 & 2.3 \\
$\bullet \quad$ with current prescription for anti-epileptic & 53 & 59.6 \\
Active patients 65 and older with hypertension & 2775 & 70.6 \\
$\bullet \quad$ with current prescription for anti-hypertensive & 1508 & 54.3 \\
Active patients 65 and older with hypotension & 189 & 4.8 \\
$\bullet \quad$ with current prescription for hypotension & 0 & 0.0 \\
Active patients 65 and older with osteoporosis & 541 & 13.8 \\
$\bullet \quad$ with current prescription for osteoporosis & 224 & 41.4 \\
Active patients 65 and older with Parkinson's disease & 54 & 1.4 \\
$\bullet \quad$ with current prescription for Parkinson's disease & 38 & 70.4 \\
Active patients 65 and older with rheumatoid arthritis & 107 & 2.7 \\
$\bullet \quad$ with current prescription for rheumatoid arthritis & 35 & 32.7 \\
\hline
\end{tabular}

Documented falls risk assessments were identified using data from multiple areas of the EHR. These areas are: 1) CPT coding; 2) Medcin findings; and 3) free text notes. Table 3.7 provides information on the counts of patients with documented falls risk assessments according to each search method, the numbers of patients added in each consecutive data step, total unduplicated counts for each, and indication of prevalence for each among patients 65 and older. Noteworthy, only 23 patients $(0.6 \%)$ have documentation of a falls risk assessment in their 
medical records at some point in the past. CPT coding alone missed $26.1 \%$ of all falls risk assessments. Value added by free text notes alone is $13.0 \%$ of all assessments. Further, only two patient records $(0.05 \%)$ have indication of having received anticipatory falls guidance at any time. Both of those instances were located in semi-structured Medcin findings. Neither of these patients have documentation of having had fallen. Appendix $G$ provides information on the specific text strings used to search for these metrics.

Table 3.7

Counts of patients with documented falls risk assessments by search method

\begin{tabular}{|c|c|c|c|c|c|}
\hline $\begin{array}{c}\text { CPT } \\
\text { coding }\end{array}$ & Medcin finding & Notes & $\begin{array}{l}\text { Undup } \\
\text { Count }\end{array}$ & $\begin{array}{c}\% \text { missed } \\
\text { CPT } \\
\text { coding } \\
\text { alone }\end{array}$ & $\begin{array}{c}\% \text { Patients } \\
65+\end{array}$ \\
\hline
\end{tabular}

\begin{tabular}{crrrrrrrr} 
Measure & Total & Total & Added & Total & Added & & & \\
Falls risk & 17 & 20 & 3 & 10 & 3 & 23 & 26.1 & 0.6 \\
assessment & 17 & & & & & & & \\
\hline
\end{tabular}

\subsection{Discussion}

This study supports the development of a novel methodology for repurposing EHR data to identify older patients at-risk for falls for the purpose of early identification of risk and efforts toward prevention. Further, findings from this study draw attention to the need for increased emphasis on falls prevention during routine office visits. Among the 3,933 patients 65 and older, only 133 patients $(3.4 \%)$ have an indication in their medical records of having had an unintentional fall at some point in the past. Searching the free text data was vital to finding even this low number of patients, as $33.8 \%$ were identified using free text searches. Given the national statistic that falls occur among approximately $40 \%$ of adults 65 and older, we can be confident that falls are underreported and/or under-documented in this sample. Likewise, falls risk assessments were sparse with only 23 patients (0.6\%) with documentation of a falls risk 
assessment in their medical records at some point in the past. As with falls, locating falls risk assessments in the EHR data was largely dependent on semi-structured and free text data. CPT coding alone missed $26.1 \%$ of all falls risk assessments. While this study is based on one EHR only, the implications for more thoroughly accounting for multiple data types when searching for clinical information are important for quality data needed for population health management, quality of care improvement, and practice based research.

This study draws attention to a multifaceted problem with falls identification in this sample of outpatient clinics. While there is an issue of low documentation of falls, this is combined with documentation practices which make it difficult to retrieve those data which are recorded. This research highlights a complex problem deserving of targeted quality improvement efforts and practice-based research. While reporting of data and benchmarking regarding unintentional falls is receiving some attention by the Physician Quality Reporting System and the National Quality Forum, health conditions and metrics more commonly measured, such as diabetes, hypertension, vitals, and patient demographics, were by far more commonly documented among this sample of clinics. While duration of EHR use may be a factor, all clinics in this study have used an EHR for at least a six year period.

\subsubsection{Limitations}

One primary limitation of this study is that purposive sampling is used to identify primary care organizations for inclusion, thereby decreasing the generalizability of the findings. Second, this study focuses on intrinsic, biologic/behavioral falls risk factors and not extrinsic, environmental risk factors due to the type of data afforded through the EHR. Combining data made available from EHRs with data sources offering extrinsic information would be beneficial. Third, this study is subject to limitations in the documentation of EHR data such as miscoding, 
missing falls data, and gaps in data due to limited sharing of information from hospitals, physical rehabilitation, and other care locations where falls information may have been recorded. Further, in terms of medications data, the EHR data exports offer medication information through the brand and generic names of the medications, as opposed to coding schema, which decreases the ability to match.

\subsection{Conclusions}

This expanded use of EHR data increases opportunity to transform data collected at the time of patient care into knowledge that can be applied to better target services and intervention to patients in need, inform health care decisions, and bolster practice-based research (Okun, et al., 2013). Further, this approach offers the advantage of moving from an acute model of patientby-patient screening to one of a planned, population model of data-driven clinical decision support for falls risk identification.

The strength of this study in its current form is one of practical importance to public health: facilitating the identification of a sector of the patient population at increased risk for falls in a way which is efficient and data-driven given the health care demands of primary care. For EHR data to be most useful to not only unintentional falls but any health condition or injury, issues of data quality, format, and accessibility need to be addressed. (Mendes \& Rodrigues, 2011). Recognizing the limits in EHR data and developing steps or interventions to improve those data are paramount to not only health informatics but to patient care and outcomes. 
Chapter 4 


\section{Chapter 4}

\section{An electronic health record data-driven model for identifying older adults at-risk for unintentional falls}

\subsection{Introduction}

Unintentional falls among older adults are multi-causal, resulting from an interaction of diverse risk factors (American Geriatrics Society, 2001). Currently, the Timed Get-Up-and-Go test is the only screening tool recommended by the USPSTF for determining risk for falls (Moyer, 2012). However, the frequency of use of this test in primary care remains low given time constraints of brief office visits (Boyd, et al., 2005). Effective care coordination and population-level management requires timely communication of clinical information (National Committee for Quality Assurance, 2013). Applying EHR data as clinical decision support in falls risk identification may serve as a means for efficient, systematic screening and support efforts in identifying at-risk older adults. Further, this use of data could help bolster use of the Timed GetUp-and-Go test by proactively identifying patients apt for screening and targeting efforts specifically to those patients.

Given the absence of published studies on using EHR data as clinical decision support in the identification of older adults at-risk for falls, this study aims to build and internally validate an EHR data-driven case finding model for use in identifying at-risk patients. A validated model would help advance the field of falls prevention through novel use of EHR data, while facilitating care coordination and population- level management of falls risk among older patients. This repurposing of EHR data can also support Meaningful Use of EHR data, specifically Stage 3 to be achieved by 2016 which gives focus to enhanced clinical decision support and improved population health (Office of the National Coordinator for Health 
Information Technology, 2015), while supporting increased capacity of primary care to repurpose data for quality improvement, practice-based research, and public health initiatives.

\subsection{Methods}

The de-identified EHR data used in this analysis are initially, by nature of the source of the data and the way in which the data were exported from the EHRs, organized in a relational database schema. That said, each type of data (i.e., patient demographics, health condition, medications, services provided, and visit/vitals information) are held in their own respective tables. These tables are linked by two unique identifiers per patient record: 1) an auto-identifier; 2) a clinic code to ensure that potential duplicate auto-identifiers across sites were able to be accounted for and distinguished. For logistic regression analysis using JMP, the data tables were collapsed into a composite flat file format using Microsoft Access queries. Adhering to the Safe Harbor Method for data de-identification, dates of service are recorded as time intervals from the first visit date documented for each patient. Days in whole numbers are used as the relative time interval. In regards to vitals data, we find:

- 557 patient records with missing heights at last visit date. Given that, the most recent documented height was used to fill-in data for 242 of those records. The remaining 315 patient heights are treated as missing data.

- 301 patient records with missing weights at last visit date. Given that, the most recent documented weight was used to fill-in data for 182 of those records. The remaining 119 patient weights are treated as missing data.

- 103 patients with missing systolic and diastolic blood pressure readings at last visit date.

Given that, the most recent documented blood pressure readings were used to fill-in data 
for 53 of those records. The remaining 50 patient systolic and diastolic blood pressure readings are treated as missing data.

- Given the potential for height, weight, BMI, and blood pressure to be associated with an unintentional fall, four additional variables were created which take into account the most proximal result for each of these metrics relative to the date of the last documented fall. Appendix $\mathrm{K}$ lists all variables included in the final data set, definitions for those variables, as well as their data types, modeling types, and value labels. All data stem from two primary care organizations, representing nine clinical sites, using the same Certification Commission for Healthcare Information Technology certified EHR.

Criteria used to identify falls risk reflect current falls prevention guidelines presented in a systematic review from current USPSTF guidelines and meta-analysis on falls risk factors among community-dwelling older adults (Deandrea, et al., 2010; Michael, et al., 2010). Key variables of interest are: being greater than or equal to 65 years of age; being female; gait or balance impairment; having a history of falls; fear of falling; vision impairment; hearing impairment; diagnosis of Parkinson's disease; dizziness/vertigo; cognitive impairment; use of a walking aid or device; current prescription for a sedative medication; current prescription for an anti-epileptic medication; current prescription for an antihypertensive medication; and currently taking four or more medications, known as polypharmacy. These variables coincide with data elements commonly collected in EHRs.

\subsubsection{Participants}

The study is accomplished by using the de-identified EHR data developed in Aim 2. This study is a nonexperimental retrospective analysis of de-identified EHR data from two primary care center organizations, representing nine physical locations excluding school-based health 
centers and dental clinics, partnering with OHSR. These centers are part of a larger network of primary care centers in the state strategically positioned in medically underserved areas (Kaiser Family Foundation, 2013; Rural Assistance Center, 2013).

Purposive sampling is used to identify primary care organizations for inclusion. Inclusion criteria are: 1) established partnership and de-identified EHR data sharing with OHSR; and 2) use of an EHR which allows for export of the necessary data. De-identified data sharing from these centers to OHSR is made possible through signed business associate agreements and memoranda of understanding. This study was classified as non-human subjects research by the WVU Office of Research Integrity and Compliance (protocol number 1402217616) as it involves secondary data that do not include Health Insurance Portability and Accountability Act protected information.

\subsubsection{Measures}

This study aims to build and internally validate an EHR data-driven case finding model for use in identifying older patients at-risk for falls based on current USPSTF guidelines and meta-analysis. There are three categories of modifiable risk factors associated with falls among older adults: biological, behavioral, and environmental (Stevens \& Schuster, 2013). The primary risk criteria for falls included in this study are biological and behavioral in nature (i.e., intrinsic) as these data elements are intrinsic to the individual and more apt to be gleaned from EHR data. Key variables of interest are: being greater than or equal to 65 years of age; being female; gait or balance impairment; having a history of falls; fear of falling; vision impairment; hearing impairment; diagnosis of Parkinson's disease; dizziness/vertigo; cognitive impairment; use of a walking aid or device; current prescription for a sedative medication; current prescription for an anti-epileptic medication; current prescription for an antihypertensive medication; and currently 
taking four or more medications, also known as polypharmacy (Deandrea, et al., 2010; Michael, et al, 2010). Extended variables of interest are: race (Non-White; White); ethnicity (Hispanic; Non-Hispanic); insurance source (Public; Private); hypertension; diabetes type 1; diabetes type 2; diabetic neuropathy; diabetic retinopathy; osteoporosis; hypotension; dementia; rheumatoid arthritis; epilepsy; muscle weakness; falls assessment; and falls guidance (Freeman, Muñoz, Rubin, \& West, 2007; Maurer, Burcham, \& Cheng, 2004).

\subsubsection{Analysis}

Analysis of the demographic characteristics, health profile, services received, and medication records of the patient population were performed by univariate analysis. Independent samples t-tests and tests of independence were used to examine potential associations across variables, in particular in relation to documented falls. Nominal logistic regression analysis with accompanying ROC analysis was used to examine the collective associations of priority and extended measures in regards to documented falls among this patient population. All analyses were completed using JMP Pro version 11.0.

\subsection{Results}

Univariate statistics were generated on patient demographics, health profile, medications, and services (Table 4.1). Results are presented in highest to lowest rank order for each data type. While these statistics were also generated in Aim 2, those statistics were derived from a relational database compared to the flat file format used in this aim. Comparison of results between analyses reveals no discrepancies, helping to validate the internal validity of the data post flat file transformation. Appendix I lists all variables included in the final data set, definitions for those variables, as well as their data types, modeling types, and value labels. 
Table 4.1

Demographics, health profile, medications, and services data for active patients age 65 and older by falls status and overall

\begin{tabular}{|c|c|c|c|c|c|c|c|}
\hline & & \multicolumn{2}{|c|}{$\begin{array}{c}\text { Patients with } \\
\text { documented falls }\end{array}$} & \multicolumn{2}{|c|}{$\begin{array}{l}\text { Patients without } \\
\text { documented falls }\end{array}$} & \multicolumn{2}{|c|}{ Total } \\
\hline & & Number & Percent & Number & Percent & Number & Percent \\
\hline \multicolumn{2}{|c|}{$\begin{array}{l}\text { Active patients age } 65 \text { and older } \\
\text { Demographics }\end{array}$} & 133 & 3.4 & 3800 & 96.6 & 3,933 & 100.0 \\
\hline \multirow[t]{2}{*}{ Age } & $65-84$ & 101 & 2.6 & 3411 & 86.7 & 3,512 & 89.3 \\
\hline & 85 and older & 32 & 0.8 & 389 & 9.9 & 421 & 10.7 \\
\hline \multirow[t]{2}{*}{ Gender } & Female & 97 & 2.5 & 2314 & 58.8 & 2,411 & 61.3 \\
\hline & Male & 36 & 0.9 & 1486 & 37.8 & 1,522 & 38.7 \\
\hline \multirow[t]{2}{*}{ Race } & White & 129 & 3.3 & 3636 & 92.4 & 3,765 & 95.7 \\
\hline & Non- White & 4 & 0.1 & 164 & 4.2 & 168 & 4.3 \\
\hline \multirow[t]{3}{*}{ Ethnicity } & $\begin{array}{l}\text { Not } \\
\text { Hispanic/Latino }\end{array}$ & 133 & 3.4 & 3766 & 95.8 & 3,899 & 99.1 \\
\hline & Hispanic/Latino & 0 & 0.0 & 27 & 0.7 & 27 & 0.7 \\
\hline & $\begin{array}{l}\text { Unreported/Refused } \\
\text { to report }\end{array}$ & 0 & 0.0 & 7 & 0.2 & 7 & 0.2 \\
\hline \multirow{2}{*}{$\begin{array}{l}\text { Insurance } \\
\text { source }\end{array}$} & Public & 97 & 2.5 & 2658 & 67.6 & 2,755 & 70.0 \\
\hline & Private & 36 & 0.9 & 1142 & 29.0 & 1,178 & 30.0 \\
\hline \multicolumn{8}{|c|}{ Health profile } \\
\hline \multicolumn{2}{|c|}{ Polypharmacy } & 127 & 3.2 & 3216 & 81.8 & 3,343 & 85.0 \\
\hline \multicolumn{2}{|c|}{ Hypertension } & 109 & 2.8 & 2666 & 67.8 & 2,775 & 70.6 \\
\hline \multicolumn{2}{|c|}{ Diabetes type 2} & 52 & 1.3 & 1140 & 29.0 & 1,192 & 30.3 \\
\hline \multicolumn{2}{|c|}{ Dizziness/Vertigo } & 37 & 0.9 & 577 & 14.7 & 614 & 15.6 \\
\hline \multicolumn{2}{|c|}{ Osteoporosis } & 35 & 0.9 & 506 & 12.9 & 541 & 13.8 \\
\hline \multicolumn{2}{|c|}{ Hearing impairment } & 27 & 0.7 & 488 & 12.4 & 515 & 13.1 \\
\hline \multicolumn{2}{|c|}{ Vision impairment } & 29 & 0.7 & 441 & 11.2 & 470 & 11.9 \\
\hline \multicolumn{2}{|c|}{ Gait/Balance impairment } & 21 & 0.5 & 183 & 4.7 & 204 & 5.2 \\
\hline \multicolumn{2}{|c|}{ Hypotension } & 12 & 0.3 & 177 & 4.5 & 189 & 4.8 \\
\hline \multicolumn{2}{|l|}{ Dementia } & 20 & 0.5 & 150 & 3.8 & 170 & 4.3 \\
\hline \multicolumn{2}{|c|}{ History of falls } & 133 & 3.4 & 0 & 0.0 & 133 & 3.4 \\
\hline \multicolumn{2}{|c|}{ Diabetes type 1} & 9 & 0.2 & 119 & 3.0 & 128 & 3.2 \\
\hline \multicolumn{2}{|c|}{ Cognitive impairment } & 9 & 0.2 & 101 & 2.6 & 110 & 2.8 \\
\hline \multicolumn{2}{|c|}{ Rheumatiod arthritis } & 9 & 0.2 & 98 & 2.5 & 107 & 2.7 \\
\hline \multicolumn{2}{|c|}{ Diabetic neuropathy } & 5 & 0.1 & 101 & 2.6 & 106 & 2.7 \\
\hline \multicolumn{2}{|l|}{ Epilepsy } & 9 & 0.2 & 80 & 2.0 & 89 & 2.2 \\
\hline \multicolumn{2}{|c|}{ Muscle weakness } & 9 & 0.2 & 83 & 2.1 & 92 & 2.3 \\
\hline \multicolumn{2}{|c|}{ Parkinson's disease } & 2 & 0.1 & 52 & 1.3 & 54 & 1.4 \\
\hline
\end{tabular}




\begin{tabular}{|c|c|c|c|c|c|c|}
\hline Diabetic retinopathy & 2 & 0.1 & 48 & 1.2 & 50 & 1.3 \\
\hline Walking aid & 1 & 0.0 & 5 & 0.1 & 6 & 0.1 \\
\hline Fear of falling & 0 & 0.0 & 1 & 0.0 & 1 & 0.0 \\
\hline \multicolumn{7}{|l|}{ Services } \\
\hline Falls assessment & 16 & 0.4 & 4 & 0.1 & 20 & 0.5 \\
\hline Falls guidance & 0 & 0.0 & 2 & 0.1 & 2 & 0.1 \\
\hline \multicolumn{7}{|l|}{ Medications } \\
\hline Anti-hypertensive medication & 76 & 1.9 & 1674 & 42.6 & 1,750 & 44.5 \\
\hline Diabetes type 2 medication & 36 & 0.9 & 882 & 22.4 & 918 & 23.3 \\
\hline Osteoporosis medication & 32 & 0.8 & 742 & 18.9 & 774 & 19.7 \\
\hline Rheumatiod arthritis medication & 33 & 0.8 & 594 & 15.1 & 627 & 15.9 \\
\hline Anti-epileptic medication & 29 & 0.7 & 568 & 14.4 & 597 & 15.2 \\
\hline Sedative medication & 17 & 0.4 & 277 & 7.0 & 294 & 7.4 \\
\hline Vertigo medication & 16 & 0.4 & 261 & 6.6 & 277 & 7.0 \\
\hline Diabetes type 1 medication & 17 & 0.4 & 256 & 6.5 & 273 & 6.9 \\
\hline Parkinson's medication & 11 & 0.3 & 137 & 3.5 & 148 & 3.8 \\
\hline Dementia medication & 1 & 0.0 & 7 & 0.2 & 8 & 0.2 \\
\hline Hypotension medication & 0 & 0.0 & 3 & 0.1 & 3 & 0.1 \\
\hline
\end{tabular}

Univariate statistics were also generated on patient vitals data (height, weight, BMI, and blood pressure) for patients with and without documented falls (Table 4.2).

Table 4.2

Vitals data for active patients age 65 and older by falls status and overall

\begin{tabular}{|c|c|c|c|c|c|c|c|}
\hline & \multicolumn{2}{|c|}{$\begin{array}{c}\text { Patients with } \\
\text { documented falls }\end{array}$} & \multicolumn{2}{|c|}{$\begin{array}{l}\text { Patients without } \\
\text { documented falls }\end{array}$} & \multicolumn{2}{|c|}{ Total } & \multirow[b]{2}{*}{$t(p)$} \\
\hline & $\begin{array}{l}\text { Mean } \\
\text { (SD) }\end{array}$ & $\begin{array}{l}\text { Percent } \\
\text { Missing }\end{array}$ & $\begin{array}{c}\text { Mean } \\
\text { (SD) }\end{array}$ & $\begin{array}{l}\text { Percent } \\
\text { Missing }\end{array}$ & $\begin{array}{l}\text { Mean } \\
\text { (SD) }\end{array}$ & $\begin{array}{l}\text { Percent } \\
\text { Missing }\end{array}$ & \\
\hline Height (in) & $\begin{array}{l}64.5 \\
(4.2)\end{array}$ & 3.8 & $\begin{array}{l}65.5 \\
(3.9)\end{array}$ & 8.1 & $\begin{array}{l}65.4 \\
(4.0)\end{array}$ & 8.0 & $\begin{array}{l}2.76 \\
(<0.01)\end{array}$ \\
\hline Weight (lbs) & $\begin{array}{l}172.1 \\
(44.6)\end{array}$ & .75 & $\begin{array}{l}178.8 \\
(42.9)\end{array}$ & 3.0 & $\begin{array}{l}178.6 \\
(43.0)\end{array}$ & 3.0 & $\begin{array}{l}1.79 \\
(>0.05)\end{array}$ \\
\hline BMI & $\begin{array}{l}29.0 \\
(6.4)\end{array}$ & 3.8 & $\begin{array}{l}29.3 \\
(6.4)\end{array}$ & 8.4 & $\begin{array}{l}29.3 \\
(6.4)\end{array}$ & 8.3 & $\begin{array}{l}0.48 \\
(>0.05)\end{array}$ \\
\hline $\begin{array}{l}\text { Systolic blood pressure } \\
(\mathrm{mmHg})\end{array}$ & $\begin{array}{l}130.7 \\
(29.9)\end{array}$ & 0.0 & $\begin{array}{l}130.1 \\
(17.3)\end{array}$ & 1.3 & $\begin{array}{l}130.1 \\
(17.9)\end{array}$ & 1.3 & $\begin{array}{l}0.35 \\
(>0.05)\end{array}$ \\
\hline $\begin{array}{l}\text { Diastolic blood pressure } \\
(\mathrm{mmHg})\end{array}$ & $\begin{array}{l}73.3 \\
(17.8)\end{array}$ & 0.0 & $\begin{array}{l}73.9 \\
(10.2)\end{array}$ & 1.3 & $\begin{array}{l}73.9 \\
(10.6)\end{array}$ & 1.3 & $\begin{array}{l}0.67 \\
(>0.05)\end{array}$ \\
\hline
\end{tabular}


Given the potential for height, weight, BMI, and blood pressure to be associated with an unintentional fall, four additional variables were created which take into account the most proximal result for each of these metrics relative to the date of the last documented fall. Results for height, weight, BMI, and blood pressure for patient with documentation of falls versus patient with no documentation of falls were analyzed using independent-samples t-tests. Analysis reveals the following:

- Significant difference in height between the two groups: $t(3618)=2.76 ; p<0.01$. Sample means illustrate that patients without documented falls are significantly taller than patients with documented falls (for non-fallers, Mean $=65.5, \mathrm{SD}=3.9$; for fallers, Mean $=64.5, \mathrm{SD}=4.2$ ). The observed difference between means was 1.0 , and the $95 \%$ confidence interval for the difference between means extended from 0.28 to 1.68 . We reject the null hypothesis that there is no difference in height between patients with and without documented falls. However, the effect size was computed as $d=-0.1$ representing a weak effect.

- Non-significant difference in weight between the two groups: $t(3816)=1.79 ; p>0.05$. Sample means illustrates that patients with and without documented falls demonstrate similar mean weights (for non-fallers, Mean $=178.9, \mathrm{SD}=42.9$; for fallers, Mean $=$ 172.1, $\mathrm{SD}=44.6)$. The observed difference between means was 6.8 , and the $95 \%$ confidence interval for the difference between means extended from -0.7 to 14.3 . We fail to reject the null hypothesis that there is no difference in weight between patients with and without documented falls.

- Non-significant difference in BMI between the two groups: $t(3605)=0.48 ; p>0.05$. Sample means illustrates that patients with and without documented falls demonstrate 
similar mean BMI (for non-fallers, Mean $=29.3, \mathrm{SD}=6.4$; for fallers, Mean $=6.4, \mathrm{SD}=$ 6.4). The observed difference between means was 0.0 , and the $95 \%$ confidence interval for the difference between means extended from -0.9 to 1.4 . We fail to reject the null hypothesis that there is no difference in BMI between patients with and without documented falls.

- Non-significant difference in systolic blood pressure between the two groups: $t(3881)=-$ $0.35 ; p>0.05$. Sample means illustrates that patients with and without documented falls demonstrate similar mean systolic blood pressure results (for non-fallers, Mean $=130.2$, $\mathrm{SD}=17.3$; for fallers, Mean $=130.7, \mathrm{SD}=29.9$ ). The observed difference between means was 0.5 , and the $95 \%$ confidence interval for the difference between means extended from -3.6 to 2.5 . We fail to reject the null hypothesis that there is no difference in systolic blood pressure between patients with and without documented falls.

- Non-significant difference in diastolic blood pressure between the two groups: $t(3881)=$ $0.67 ; p>0.05$. Sample means illustrates that patients with and without documented falls demonstrate similar mean diastolic blood pressure results (for non-fallers, Mean =73.9, $\mathrm{SD}=10.2$; for fallers, Mean $=73.3, \mathrm{SD}=17.8$ ). The observed difference between means was 0.6 , and the $95 \%$ confidence interval for the difference between means extended from -1.2 to 2.5 . We fail to reject the null hypothesis that there is no difference in systolic blood pressure between patients with and without documented falls.

Chi-square tests of independence were performed to examine the relation between falls and the priority and extended variables in an unadjusted sense. Table 4.3 displays these results. In regards to the priority variables, we can reject the null hypothesis and conclude that the following variables are related to falls: age category (85 and older; 65-84); gender (female; 
male); gait/balance impairment; vision impairment; hearing impairment; dizziness/vertigo; cognitive impairment; sedative medication; anti-epileptic medication; anti-hypertension medication; and polypharmacy. In regards to the extended variables, we can reject the null hypothesis and conclude that the following variables are related to falls: hypertension; type 2 diabetes; type 1 diabetes; osteoporosis; hypotension; dementia; rheumatoid arthritis; epilepsy; muscle weakness; and falls assessment.

Table 4.3

Chi-square tests of independence for falls

\begin{tabular}{llllll}
\hline & \multicolumn{1}{c}{$\chi^{2}$} & \multicolumn{1}{c}{$P$} & \multicolumn{1}{c}{$O R$} & $\begin{array}{c}\text { Lower 95\% } \\
\text { CI }\end{array}$ & $\begin{array}{c}\text { Upper } \\
95 \% \text { CI }\end{array}$ \\
\cline { 2 - 6 } Priority measures & & & & \\
\hline Age category (85 and older; 64-85) & 25.69 & $<0.00^{* * * *}$ & 0.36 & 0.24 & 0.54 \\
Gender (Female; Male) & 7.85 & $0.01^{*}$ & 0.58 & 0.39 & 0.85 \\
Gait/Balance impairment & 31.47 & $<0.00^{* * * *}$ & 3.71 & 2.27 & 6.04 \\
Vision impairment & 12.70 & $0.00^{* * *}$ & 2.12 & 1.39 & 3.24 \\
Hearing impairment & 6.28 & $0.01^{*}$ & 1.73 & 1.12 & 2.67 \\
Parkinson's disease & 0.017 & 0.90 & 1.10 & 0.27 & 4.57 \\
Dizziness/Vertigo & 15.57 & $<0.00^{* * * *}$ & 2.15 & 1.46 & 3.18 \\
Cognitive impairment & 7.98 & $0.00^{*}$ & 2.66 & 1.31 & 5.38 \\
Walking aid & 3.25 & 0.07 & 5.75 & 0.67 & 49.56 \\
Sedative medication & 5.61 & $0.02^{*}$ & 1.86 & 1.10 & 3.15 \\
Anti-epileptic medication & 4.69 & $0.03^{*}$ & 0.63 & 0.41 & 0.96 \\
Anti-hypertension medication & 8.92 & $0.00^{* *}$ & 1.69 & 1.19 & 2.40 \\
Polypharmacy & 11.88 & $0.00^{* * *}$ & 3.84 & 1.69 & 8.76 \\
Fear of falling & 0.035 & 0.85 & 0.00 & $\cdot$ &. \\
Extended measures & & & & & \\
\hline Race (Non-White; White) & 0.54 & 0.46 & 0.69 & 0.25 & 1.88 \\
Ethnicity (Hispanic; Non-Hispanic) & 0.95 & 0.33 & 0.00 &. &. \\
Insurance source (Public; Private) & 0.55 & 0.46 & 1.16 & 0.78 & 1.71 \\
Hypertension & 8.61 & $0.00^{* *}$ & 1.93 & 1.24 & 3.02 \\
Diabetes type 2 & 5.04 & $0.02^{*}$ & 1.50 & 1.05 & 2.14 \\
Osteoporosis & 18.31 & $<.00^{* * * *}$ & 2.32 & 1.56 & 3.46 \\
Hypotension & 5.351 & $0.02^{*}$ & 2.03 & 1.10 & 3.74 \\
Dementia & 38.22 & $<.00^{* * * *}$ & 4.31 & 2.61 & 7.12 \\
Diabetes type 1 & 5.39 & $0.02^{*}$ & 2.25 & 1.11 & 4.52 \\
Rheumatiod arthritis & 8.52 & $0.00^{* *}$ & 2.74 & 1.35 & 5.55 \\
Diabetic neuropathy & 0.60 & 0.44 & 1.43 & 0.57 & 3.57 \\
Epilepsy & 12.63 & $0.00^{* * *}$ & 3.38 & 1.66 & 6.88 \\
Muscle weakness & 11.81 & $0.00^{* * *}$ & 3.25 & 1.60 & 6.62
\end{tabular}


Diabetic retinopathy

Falls assessment

$0.06 \quad 0.81$

$361.18<.00 * * * *$

1.19

0.29

4.96

$\begin{array}{lccc}\text { Falls guidance } & 0.07 & 0.79 & 0\end{array}$. measures except Ethnicity, $N=3926$ and $d f=1$.

\subsubsection{Model 1: priority falls risk variables}

Nominal logistic regression analysis was performed on all priority falls risk variables as identified by the USPSTF guidelines and meta-analysis on falls risk factors among communitydwelling older adults (Deandrea, et al., 2010; Michael, et al., 2010). Only one patient record had documentation of fear of falling, therefore for the reliability of the model that variable was excluded. The variables included in the Model 1: being greater than or equal to 65 years of age; being female; gait or balance impairment; having a history of falls; vision impairment; hearing impairment; diagnosis of Parkinson's disease; dizziness/vertigo; cognitive impairment; use of a walking aid or device; current prescription for a sedative medication; current prescription for an anti-epileptic medication; current prescription for an antihypertensive medication; and currently taking four or more medications, also known as polypharmacy. All 3,933 patient records, 133 of which have documentation of a fall, were included as there were no missing data across the priority variables for any case.

A test of the full model with all predictors against a constant-only model was statistically significant, $\chi^{2}(13, N=3,933)=67.43, p<0.0001$, indicating that the predictors, as a set, reliably distinguish between patients who have documentation of a history of falls and those who do not. Table 4.4 provides the chi-square values and indication of significance, odds ratio results, and lower and upper $95 \%$ confidence intervals for each of the predictor variables for Model 1. Chisquare results indicate that only four variables reliably predict falls status: age category $\chi^{2}(1, N$ $=3,933)=10.47, p<0.01$; gait/balance impairment $\chi^{2}(1, N=3,933)=5.18, p<0.05$; 
dizziness/vertigo $\chi^{2}(1, N=3,933)=3.88, p<0.05$; and polypharmacy $\chi^{2}(1, N=3,933)=5.48$, $p<0.05$. For the significantly associated variables, odds ratio results indicate the following:

- patients age 85 and older have 2.1 times higher odds for documentation of falls compared to patients age 65 to 84 controlling for all variables in the model, 95\% CI [1.37, 3.27];

- patients with documentation of gait/balance impairment have 1.9 times higher odds for documentation of falls compared to patients without documentation of gait/balance impairment controlling for all variables in the model, 95\% CI [1.07, 3.28];

- patients with documentation of dizziness/vertigo have 1.53 times higher odds for documentation of falls compared to patients without documentation of dizziness/vertigo controlling for all variables in the model, however the $95 \%$ confidence interval indicates a non-signific ant relationship, 95\% CI [1.00, 2.28];

- patients with polypharmacy have 2.4 times higher odds for documentation of falls compared to patients without documentation of polypharmacy controlling for all variables in the model, $95 \% \mathrm{CI}[1.12,6.30]$. Noteworthy, planned refinements to this analysis will account for potential multicollinearity between polypharmacy and medication classes included in this model, taking into account the average number of active medications per patient, to enrich information on this particular patient population. ROC analysis indicates a relatively poor ability of the model to discriminate between patients with documentation of falls and those without documentation of falls (AUC $=0.69)$. Model fit statistics indicate $\mathrm{AICc}=1123.02$ and $\mathrm{BIC}=1210.80$ (Figure 4.1). 
Table 4.4

Nominal logistic regression results - Model 1 with all priority risk variables

\begin{tabular}{llllll}
\hline & \multicolumn{1}{c}{$\chi^{2}$} & $P$ & & OR & \multicolumn{2}{c}{ Lower } & \multicolumn{2}{c}{ Upper 95\% } \\
Age category (85 and older; 64-85) & 10.74 & $0.00^{* *}$ & 2.15 & 1.37 & 3.27 \\
Gender (Female; Male) & 3.65 & 0.06 & 1.46 & 0.99 & 2.20 \\
Gait/Balance impairment & 4.78 & $0.03^{*}$ & 1.91 & 1.07 & 3.28 \\
Vision impairment & 3.37 & 0.07 & 1.55 & 0.97 & 2.40 \\
Hearing impairment & 0.84 & 0.36 & 1.25 & 0.77 & 1.97 \\
Parkinson's disease & 0.27 & 0.61 & 0.69 & 0.11 & 2.38 \\
Dizziness/Vertigo & 3.89 & $0.04^{*}$ & 1.53 & 1.00 & 2.28 \\
Cognitive impairment & 0.64 & 0.42 & 1.38 & 0.61 & 2.79 \\
Walking aid & 0.50 & 0.48 & 2.43 & 0.12 & 17.39 \\
Sedative medication & 0.33 & 0.56 & 1.18 & 0.65 & 2.01 \\
Anti-epileptic medication & 0.46 & 0.50 & 0.86 & 0.56 & 1.36 \\
Anti-hypertension medication & 2.34 & 0.13 & 1.33 & 0.92 & 1.91 \\
Polypharmacy & 5.11 & $0.02^{*}$ & 2.41 & 1.11 & 6.30 \\
\hline
\end{tabular}

Note. $*=p<0.05, * *=p<0.01, N=3933$, and $d f=1$ for all analyses.

Figure 4.1

ROC results - Model 1 with all priority risk variables $(A U C=0.69)$

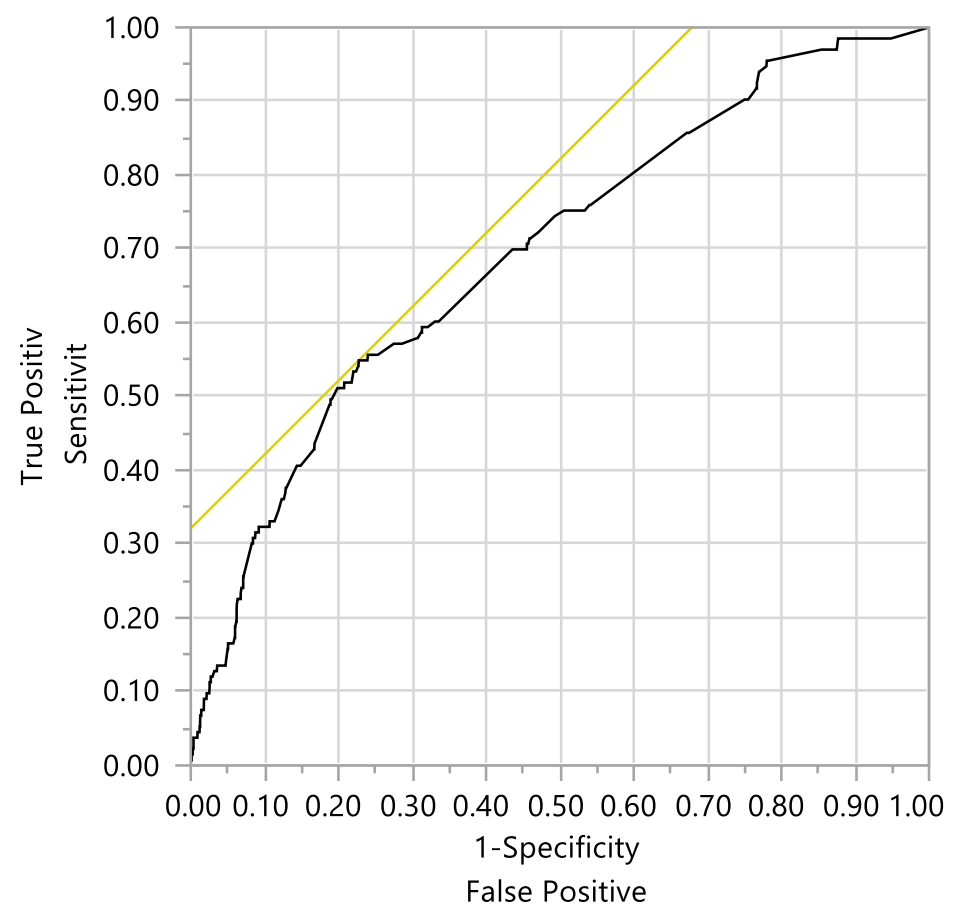




\subsubsection{Model 2:extended falls risk variables}

Model 2 was built to evaluate only the extended set of falls risk variables (i.e., those indicated in literature as important potential associations with unintentional falls but not highlighted by the USPSTF or recent systematic review as priority indicators). Variables included in the Model 2 are: race (Non-White; White); ethnicity (Hispanic; Non-Hispanic); insurance source (Public; Private); hypertension; diabetes type 1; diabetes type 2; osteoporosis; hypotension; dementia; rheumatiod arthritis; diabetic neuropathy; epilepsy; muscle weakness; diabetic retinopathy; falls assessment; and falls guidance. Race, ethnicity, and insurance status were added to the model to examine potential demographic factors. This model was found to be unstable due to low counts of patients receiving falls guidance and low counts of patients who are Hispanic. Given this, the variables ethnicity and falls guidance were removed from the model. The resulting model was statistically significant, $\chi^{2}(14, N=3,933)=160.64, p<0.001$, indicating that the predictors, as a set, reliably distinguish between patients who have documentation of a history of falls and those who do not. Table 4.5 provides the chi-square values and indication of significance, odds ratio results, and lower and upper $95 \%$ confidence intervals for each of the predictor variables. Chi-square results indicate that the following variables reliably predict falls status: osteoporosis $\chi^{2}(1, N=3,933)=8.00, p<0.05$; dementia $\chi$

${ }^{2}(1, N=3,933)=20.50, p<0.0001$; rheumatoid arthritis $\chi^{2}(1, N=3,933)=5.95, p<0.05$;

epilepsy $\chi^{2}(1, N=3,933)=3.98, p<0.05$; muscle weakness $\chi^{2}(1, N=3,933)=5.24, p<0.05$;

and falls assessment $\chi^{2}(1, N=3,933)=97.57, p<0.0001$. For the significantly associated variables, odds ratio results indicate the following: 
- patients with documentation of osteoporosis have 1.91 times higher odds for documentation of falls compared to patients without documentation of osteoporosis controlling for all variables in the model, 95\% CI [1.23, 2.92];

- patients with documentation of dementia have 3.96 times higher odds for documentation of falls compared to patients without documentation of dementia controlling for all variables in the model, $95 \% \mathrm{CI}[2.27,6.61]$;

- patients with documentation of rheumatoid arthritis have 2.76 times higher odds for documentation of falls compared to patients without documentation of rheumatoid arthritis controlling for all variables in the model, 95\% CI [1.24, 5.46];

- patients with documentation of epilepsy have 2.38 times higher odds for documentation of falls compared to patients without documentation of epilepsy controlling for all variables in the model, $95 \% \mathrm{CI}[1.02,4.95]$;

- patients with documentation of muscle weakness have 2.62 times higher odds for documentation of falls compared to patients without documentation of muscle weakness controlling for all variables in the model, 95\% CI [1.16, 5.26];

- patients with documentation of having received a falls risk assessment have 182.68 times higher odds for documentation of falls compared to patients without documentation of having received a falls risk assessment controlling for all variables in the model, $95 \% \mathrm{CI}$ $[63.67,662.98]$.

ROC analysis indicates a fair ability of the model to discriminate between patients with documentation of falls and those without documentation of falls $(\mathrm{AUC}=0.75)$. Model fit statistics indicate $\mathrm{AICc}=1031.82, \mathrm{BIC}=1125.86$ (Figure 4.2). 
Table 4.5

Nominal logistic regression results - Model 2 with all extended falls risk variables

\begin{tabular}{|c|c|c|c|c|c|}
\hline & $\chi^{2}$ & $P$ & OR & $\begin{array}{l}\text { Lower } \\
95 \% \text { CI }\end{array}$ & $\begin{array}{c}\text { Upper } 95 \% \\
\text { CI }\end{array}$ \\
\hline Race (Non-White; White) & $\overline{1.77}$ & 0.18 & 0.49 & 0.13 & 1.35 \\
\hline Insurance source (Public; Private) & 0.19 & 0.66 & 1.10 & 0.73 & 1.70 \\
\hline Hypertension & 1.96 & 0.16 & 1.40 & 0.88 & 2.33 \\
\hline Diabetes type 1 & 1.50 & 0.22 & 1.66 & 0.72 & 3.44 \\
\hline Diabetes type 2 & 1.85 & 0.17 & 1.34 & 0.88 & 2.01 \\
\hline Osteoporosis & 8.01 & $0.01 *$ & 1.91 & 1.23 & 2.92 \\
\hline Hypotension & 1.07 & 0.30 & 1.43 & 0.71 & 2.66 \\
\hline Dementia & 20.50 & $<0.00 * * * *$ & 3.96 & 2.27 & 6.61 \\
\hline Arthritis & 5.95 & $0.01 *$ & 2.76 & 1.24 & 5.46 \\
\hline Diabetic neuropathy & 0.05 & 0.82 & 1.12 & 0.37 & 2.73 \\
\hline Epilepsy & 3.98 & $0.05^{*}$ & 2.38 & 1.02 & 4.95 \\
\hline Muscle weakness & 5.24 & $0.02 *$ & 2.62 & 1.16 & 5.26 \\
\hline Diabetic retinopathy & 0.13 & 0.72 & 0.77 & 0.12 & 2.72 \\
\hline Falls assessment & 97.57 & $<0.00 * * * *$ & 182.68 & 63.67 & 662.99 \\
\hline
\end{tabular}

Figure 4.2

ROC results - Model 2 with all extended risk variables $(A U C=0.75)$

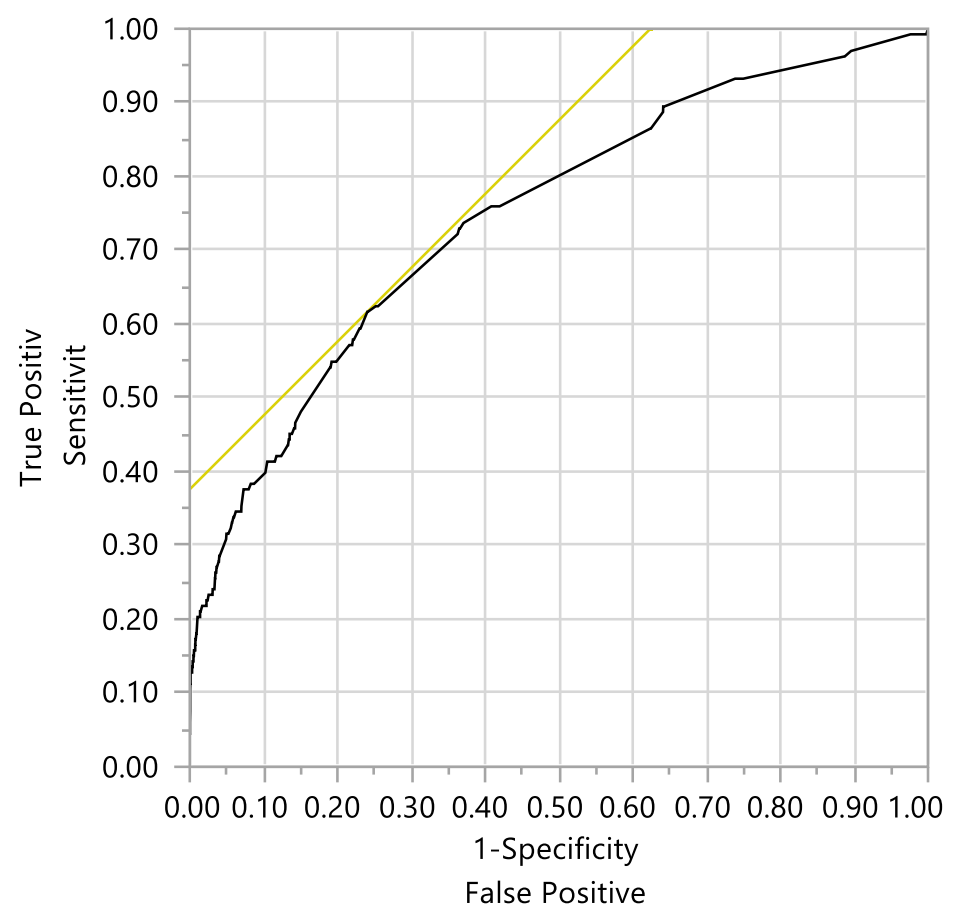




\subsubsection{Model 3:priority and extended falls risk variables}

Model 3 accounts for both the priority and extended falls risk factors examined in Models 1 and 2. That stated, the variables included in Model 3 are: being greater than or equal to 65 years of age; being female; gait or balance impairment; having a history of falls; vision impairment; hearing impairment; diagnosis of Parkinson's disease; dizziness/vertigo; cognitive impairment; use of a walking aid or device; current prescription for a sedative medication; current prescription for an anti-epileptic medication; current prescription for an antihypertensive medication; polypharmacy; race (Non-White; White); insurance source (Public; Private); hypertension; diabetes type 1; diabetes type 2; osteoporosis; hypotension; dementia; rheumatiod arthritis; diabetic neuropathy; epilepsy; muscle weakness; diabetic retinopathy; and falls assessment. The model was statistically significant, $\chi^{2}(27, N=3,933)=203.60, p<0.0001$, indicating that the predictors, as a set, reliably distinguish between patients who have documentation of a history of falls and those who do not. Table 4.6 provides the chi-square values and indication of significance, odds ratio results, and lower and upper $95 \%$ confidence intervals for each of the predictor variables in Model 3. Chi-square results indicate that the following variables in this combined model reliably predict falls status: age category $\chi^{2}(1, N=$ $3,933)=14.00, p<0.001$; gender $\chi^{2}(1, N=3,933)=5.05, p<0.05$; dementia $\chi^{2}(1, N=3,933)=$ $10.51, p<0.01$; rheumatoid arthritis $\chi^{2}(1, N=3,933)=5.62, p<0.05$; epilepsy $\chi^{2}(1, N=3,933)$ $=4.63, p<0.05$; muscle weakness $\chi^{2}(1, N=3,933)=4.52, p<0.05$, and falls assessment $\chi^{2}(1$, $N=3,933)=104.31, p<0.0001$. For the significantly associated variables, odds ratio results indicate the following: 
- patients age 85 and older have 2.58 times higher odds for documentation of falls compared to patients age 65 to 84 controlling for all variables in the model, $95 \% \mathrm{CI}$ $[1.59,4.08]$

- female patients have 1.67 times higher odds for documentation of falls compared to male patients controlling for all variables in the model, $95 \%$ CI [1.07, 2.68];

- patients with documentation of dementia have 2.91 times higher odds for documentation of falls compared to patients without documentation of dementia controlling for all variables in the model, $95 \% \mathrm{CI}[1.55,5.26]$;

- patients with documentation of rheumatoid arthritis have 2.71 times higher odds for documentation of falls compared to patients without documentation of rheumatoid arthritis controlling for all variables in the model, $95 \% \mathrm{CI}[1.21,5.43]$;

- patients with documentation of epilepsy have 2.73 times higher odds for documentation of falls compared to patients without documentation of epilepsy controlling for all variables in the model, $95 \% \mathrm{CI}[1.10,6.05]$;

- patients with documentation of muscle weakness have 2.50 times higher odds for documentation of falls compared to patients without documentation of muscle weakness controlling for all variables in the model, $95 \%$ CI $[1.08,5.18]$;

- patients with documentation of having received a falls risk assessment have 285.24 times higher odds for documentation of falls compared to patients without documentation of having received a falls risk assessment controlling for all variables in the model, 95\% CI [93.21, 1091.99]. 
ROC analysis indicates an increased ability of the model to discriminate between patients with documentation of falls and those without documentation of falls (AUC $=0.79$ ). Model fit statistics indicate $\mathrm{AICc}=1015.16, \mathrm{BIC}=1190.50$ (Figure 4.3).

Table 4.6

Nominal logistic regression results - Model 3 with priority and extended falls risk variables

\begin{tabular}{|c|c|c|c|c|c|}
\hline & $\chi^{2}$ & $P$ & $O R$ & $\begin{array}{l}\text { Lower } \\
95 \% \text { CI }\end{array}$ & $\begin{array}{c}\text { Upper } 95 \% \\
\text { CI }\end{array}$ \\
\hline Age category (85 and older; 64-85) & $\overline{14.00}$ & $0.00 * * *$ & 2.58 & 1.59 & 4.08 \\
\hline Gender (Female; Male) & 5.05 & $0.02 *$ & 1.67 & 1.06 & 2.68 \\
\hline Gait/Balance impairment & 1.13 & 0.29 & 1.40 & 0.74 & 2.53 \\
\hline Vision impairment & 3.07 & 0.08 & 1.57 & 0.94 & 2.51 \\
\hline Hearing impairment & 0.20 & 0.65 & 1.12 & 0.66 & 1.84 \\
\hline Parkinson's disease & 2.28 & 0.13 & 0.31 & 0.04 & 1.34 \\
\hline Dizziness/Vertigo & 1.33 & 0.25 & 1.31 & 0.82 & 2.03 \\
\hline Cognitive impairment & 0.01 & 0.94 & 0.97 & 0.40 & 2.10 \\
\hline Walking aid & 1.03 & 0.31 & 3.82 & 0.18 & 27.66 \\
\hline Sedative medication & 0.04 & 0.83 & 1.07 & 0.56 & 1.89 \\
\hline Anti-epileptic medication & 0.31 & 0.57 & 0.77 & 0.28 & 1.80 \\
\hline Anti-hypertension medication & 1.75 & 0.19 & 1.31 & 0.88 & 1.98 \\
\hline Polypharmacy & 2.93 & 0.09 & 2.09 & 0.91 & 5.85 \\
\hline Race (Non-White; White) & 1.97 & 0.16 & 2.15 & 0.76 & 8.34 \\
\hline Insurance source (Public; Private) & 0.30 & 0.58 & 1.13 & 0.74 & 1.76 \\
\hline Hypertension & 0.11 & 0.74 & 1.01 & 0.66 & 1.85 \\
\hline Diabetes type 1 & 0.86 & 0.35 & 1.47 & 0.63 & 3.07 \\
\hline Diabetes type 2 & 1.20 & 0.27 & 1.27 & 0.82 & 1.94 \\
\hline Osteoporosis & 2.06 & 0.15 & 1.40 & 0.88 & 2.20 \\
\hline Hypotension & 0.31 & 0.58 & 1.22 & 0.59 & 2.31 \\
\hline Dementia & 10.54 & $0.00 * *$ & 2.91 & 1.55 & 5.26 \\
\hline Arthritis & 5.62 & $0.02 *$ & 2.71 & 1.21 & 5.42 \\
\hline Diabetic neuropathy & 0.08 & 0.78 & 1.15 & 0.38 & 2.82 \\
\hline Epilepsy & 4.63 & $0.03 *$ & 2.73 & 1.10 & 6.05 \\
\hline Muscle weakness & 4.51 & $0.03 *$ & 2.50 & 1.08 & 5.18 \\
\hline Diabetic retinopathy & 1.03 & 0.31 & 0.48 & 0.07 & 1.79 \\
\hline Falls assessment & 104.31 & $<0.00 * * * *$ & 258.24 & 93.21 & 1091.99 \\
\hline
\end{tabular}


Figure 4.3

ROC results - Model 3 with priority and extended falls risk variables $(A U C=0.79)$

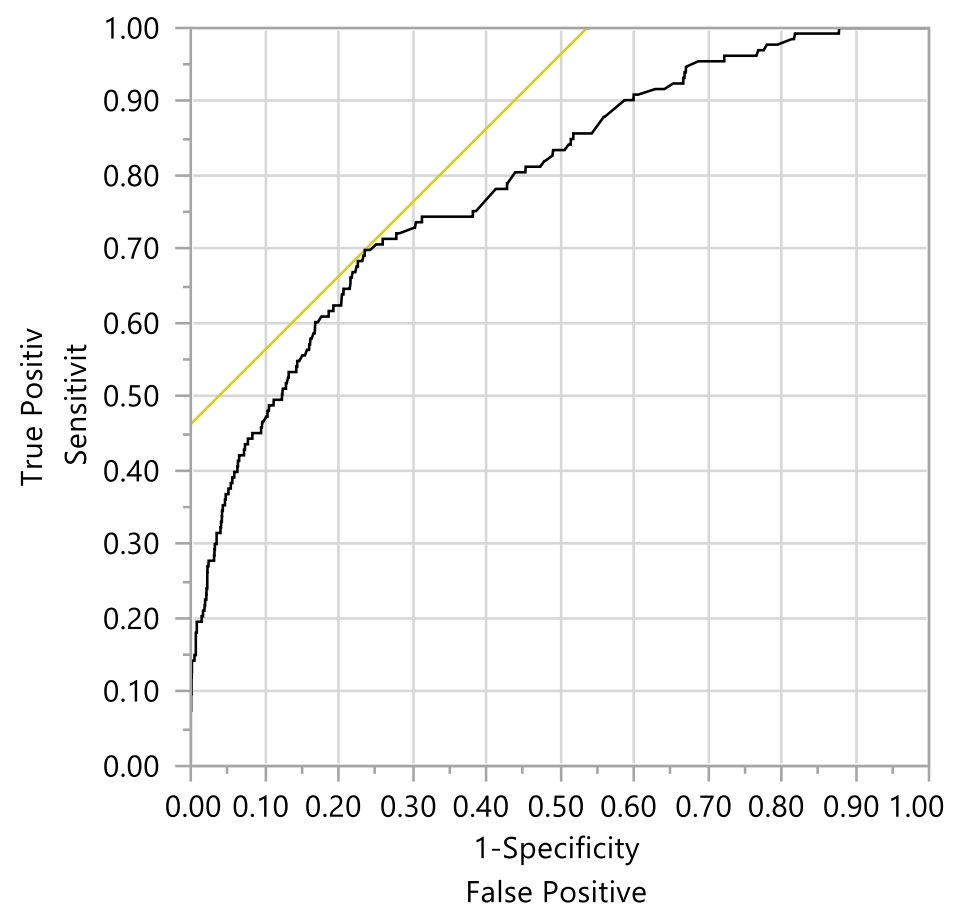

\subsection{Discussion}

The areas under the ROC curve, or AUCs, here calculated indicate how well the sets of risk variables, taken as a whole, discriminate between patients with and without documented falls. Across Models 1 through 3, we find an increased ability to make this discernment. In effect, the increase in the AUC across Models 1 through 3 are telling in terms of the value of the variables sets independently, and the greater collective value of the variables sets combined. In Model 1 in which only the priority measures were examined, we find an AUC of 0.69 which is overall weak. Comparatively, in Model 2 in which only the extended measures were examined we find an increase in the AUC to 0.75. This increase indicates, in this particular patient population, the value in looking beyond only the priority measures as identified by the USPSTF and recent systematic review to a set of secondary measures as identified by literature on falls 
among older adults. Further, in Model 3 which takes into account the priority and extended measures combined, we find an increase in the AUC to 0.79 demonstrating moderate discriminatory power and making the model more apt to be useful in a clinical setting. Further, factors included in this model are more reflective of the primary causes of falls among older adults in Appalachia, giving this approach potentially stronger clinical applications in WV.

\subsubsection{Limitations}

This analysis is subject to potential limitations in the documentation of EHR data such as miscoding, potential missing falls data, and limitations in data sharing from hospitals and other care locations where falls information may have been recorded. Additionally, this study proposed the development of a point-based algorithm to identify falls risk based on current USPSTF guidelines and meta-analysis on falls risk factors with the intent that an updated point-based ranking for falls risk would benefit the field. However, we have too few documented falls cases to reliably create such a prediction model. That stated, we can still accurately describe association among priority and extended variables in regards to documented falls. Further, this study is limited to analys is of internal validity only. Feasibility of conducting a test of external model validity was explored through a planned ROC analysis comparing patient risk identification using the gold standard Timed Get Up and Go test versus the EHR data-based risk identification model here developed (Hanley \& McNeil, 1983). The Timed Get Up and Go test has a sensitivity of $87 \%$ and specificity of $87 \%$ in identifying community-dwelling older adults at-risk for falls (Shumway-Cook, Brauer, \& Woollacott, 2000). A priori power analysis indicates need for 256 patients to have $80 \%$ power for detecting a medium sized effect using .05 criterion of statistical significance. While such an analysis is outside of the scope of the dissertation, future research building on the dissertation is planned to address external model validity. The 
strength of this study in its current form is one of practical importance to public health:

facilitating the identification of a sector of the patient population at increased risk for falls in a way which is efficient and data-driven given the health care demands of primary care.

\subsection{Conclusions}

There is value added in expanding beyond the priority falls risk factors. Repurposing EHR data allows for a broader look at falls risk factors in a way which is sensitive to the time constraints of the routine office visit and complementary to the movement in primary care to become meaningful users of EHR data. Whereas falls screenings take priority factors into account, we find that the set of extended measures is of particular importance among this patient population. In effect, this data-driven approach to falls risk identification allows for a broader scope in risk identification with increased discernment while also providing opportunity to supplement low falls risk screening rates. 
Chapter 5 


\subsection{Summary}

Unintentional falls among older adults are a complex public health problem both nationally and in WV. Across the US, nearly $40 \%$ of community-dwelling adults age 65 and older fall at least once a year making unintentional falls the leading cause of both fatal and nonfatal injuries among this age group (Michael, et al., 2010; Centers for Disease Control and Prevention, 2014). The human and financial costs of these injuries and their complications are worthy of increased public health attention. Addressing this public health problem in the primary care setting offers promise in identifying and addressing this at-risk population. However, the challenges in incorporating falls risk screening into primary care culminate in a problem of missed opportunity for screening, counseling, intervention, and ultimately prevention of falls among older adults. Given these barriers, the three studies comprising this body of research aim to better understand current falls risk screening practices in WV primary care and determine the potential for innovative use of routinely collected EHR data for enhanced clinical decision support to busy, often resource-thin primary care environments. This research is especially needed as, to date, there have been no published studies on using EHR data as clinical decision support in the identification of older adults at-risk for falls. The resultant information, methods, and tools are intended to help foster the Institute for Healthcare Improvement's Triple Aim of improved quality of patient-level care, improved health of patient populations, and decreased health care costs (Institute for Healthcare Improvement, 2013).

Focus group discussions with primary care partners serve as the cornerstone of this dissertation. These sessions were revealing in terms of helping to better understand falls risk screening practices in these sites, the impeding and promoting determinants to screening, and the potential for EHR data-based clinical decision support to be incorporated into the care setting. 
We find a significant lack of readiness to innovatively use routinely collected EHR data for population health management for falls prevention due to a myriad of environmental barriers and perceived obstacles to change. The topic of falls risk identification and prevention is a rarely discussed topic across these sites. Likewise, the extent to which older adult patients are screened for risk and referred for services is sparse at best. The four primary care centers in this study use no form of consistent, standardized screening. Furthermore, data routinely collected in EHRs such as age, demographics, diagnoses, and medications, while applicable to falls risk identification, are not viewed as actionable information. Moreover, falls-related data are not collected in standardized, well-understood ways. These findings give vital context to the EHR data examined in the later aims of this research.

Using de-identified EHR from a sample of WV primary care centers, we find that it is both feasible and worthwhile to repurpose routinely collected data for the purpose of identification of older adult patients at-risk for falls. Among the 3,933 patients 65 and older included in this research, only 133 patients $(3.4 \%)$ have an indication in their medical records of having had an unintentional fall at some point in the past. Searching the free text data was vital to finding even this low number of patients, as $33.8 \%$ were identified using free text searches. Given that nearly $40 \%$ of community-dwelling adults age 65 and older fall at least once a year, (Michael, et al., 2010; Centers for Disease Control and Prevention, 2014), our finding of 3.4\% with a history of falls underestimates the true number of falls cases. Given the focus group findings, underreporting of falls on the part of the patients and missed opportunities to learn of falls due to lack of information sharing across health care service sites are also contributing factors. Similarly, documentation of falls risk assessments were sparse with only 23 patients $(0.6 \%)$ having documentation of a falls risk assessment in their medical records at some point in 
the past. As with falls, locating falls risk assessments in the EHR data was largely dependent on semi-structured and free text data. CPT coding alone missed $26.1 \%$ of all falls risk assessments. There is clear benefit in accounting for multiple data types when searching for falls-related clinical information. Given findings from the focus group discussions on current handling of falls-related data in the EHRs, these results are not surprising.

The strengths of using routinely collected data become even more apparent when concurrently examining the collective body of priority and secondary falls risk factors. We find clear value added in expanding beyond the priority falls risk factors at identified by the USPSTF and recent systematic review. Repurposing EHR data in a population health framework allows for a broader look at falls risk factors in a way which is sensitive to the time constraints of the routine office visit and complementary to the movement in primary care to become meaningful users of EHR data. A data-driven approach to falls risk identification allows for a broader scope in risk identification with increased discernment while also providing opportunity to supplement low falls risk screening rates.

\subsection{Significance}

David Blumenthal, former National Coordinator for Health Information Technology, US Department of Health and Human Services, is quoted as saying: "Nothing is more fundamental to the future of medicine and health care than having better information that is well managed, easily accessible and timely in order to meet the needs of the US population and to improve the overall quality of care" (National Committee for Quality Assurance, 2009). Contained in that statement is a myriad of challenges and opportunities in building closer, stronger public health/primary care partnerships designed to improve key population health metrics such as unintentional falls among older adults. This dissertation, as a whole, demonstrates great need for 
enhanced mechanisms in falls screening in primary care and potential for EHR data to help facilitate those screenings. The significance of this dissertation then is one of practical importance to public health: better understanding the primary care environment as it relates to falls screening practices, collection of key metrics in EHRs, and ability to repurpose those data for supplemental clinical decision support in falls risk identification.

\subsection{Strengths and Weaknesses}

The focus group discussions in Aim 1 are based on a non-randomized sampling of WV primary care centers, making the study limited in terms of its generalizability. Further, the focus group interviews are susceptible to facilitator bias which can harm the validity and reliability of the findings (Miles \& Huberman, 1994). Facilitator bias was addressed by the primary and secondary research by avoiding opinion sharing and avoiding asking biased questions. Focus groups are also subject to both positive and negative group effects; ranging from problems due to dominant group members, unwillingness to share in a group setting, and issues in power and position (Kaplowitz \& Hoehn, 2001). However, the strength of the study is the rich, contextual information gathered on current falls screening practices, the impeding and promoting determinants to screening, and the potential for EHR data-based clinical decision support to be incorporated into the care setting. These are critical issues in planning any data-driven public health effort designed to increasing screening among older adults.

The de-identified data sets used in Aim 2 are based on purposive sampling, thereby decreasing the generalizability of the findings. Further, given the sources of the data (EHRs), we have access to intrinsic, biologic/behavioral falls risk factors but not extrinsic, environmental risk factors. We also encounter limitations in the documentation of EHR data such as miscoding, missing falls data, and gaps in data due to limited sharing of information from hospitals, physical 
rehabilitation, and other care locations where falls information may have been recorded.

However, the strength of the study is one of efficiency and innovation. We find that it is possible to repurpose EHR data to build a more comprehensive set of data by which to identify at-risk patients. These approaches are used with chronic health conditions, and can be applied to unintentional falls. While challenging, the potential public health benefits of repurposing data indicate that the effort is worthwhile.

In Aim 3, we initially proposed the development of a point-based algorithm to identify falls risk based on current USPSTF guidelines and meta-analysis on falls risk factors with the intent that an updated point-based ranking for falls risk would benefit the field. However, we have too few documented falls cases to reliably create such a prediction model. That stated, we were still able to accurately describe associations among priority and extended variables in regards to documented falls. Further, this study is limited to analysis of internal validity only. Feasibility of conducting a test of external model validity was explored through a planned ROC analysis comparing patient risk identification using the gold standard Timed Get Up and Go test versus the EHR data-based risk identification model here developed (Hanley \& McNeil, 1983). A priori power analysis indicates need for 256 patients to have $80 \%$ power for detecting a medium sized effect using .05 criterion of statistical significance. Such an analysis is outside of the scope of the dissertation. The strength of this study in its current form is one of practical importance to public health: facilitating the identification of a sector of the patient population at increased risk for falls in a way which is efficient and data-driven given the health care demands of primary care. 


\subsection{Future Research}

\subsubsection{Electronic health record data quality}

For EHR data to be most useful to not only unintentional falls risk identification and intervention but any health condition or injury, issues of data quality, format, and accessibility need to be addressed. (Mendes \& Rodrigues, 2011). Recognizing the limits in EHR data and developing steps or interventions to improve those data are paramount to not only health informatics but to patient care and outcomes. There is a complex mixture of environmental, organizational, training, and technical barriers impacting data quality. Given these challenges, future research efforts to make EHR data more usable for research and quality of care improvement are warranted. The Agency for Healthcare Research and Quality is a primary source of potential grant funds to address this issue, through the R03 small grants mechanism targeted to improving quality of care through applications of health information technology.

Interventions grounded on the sociotechnical approach to integration of health information technology are advisable. This approach emphasizes that healthcare systems be studied and best understood to inform the design, implementation and use of EHRs (Berg M., 1999; O'Carroll, Yasnoff, Ward, Ripp, \& Martin, 2003; Doolan, Bates, \& James, 2003). From the sociotechnical approach, there is no standard set of technological and/or organizational problems to be solved. Each setting poses unique difficulties in implementation (Berg M. , 1999). Improved EHR data quality, for example, is the byproduct of a thoughtful, well-planned combination of iterative modifications to traditional office procedures and flexibility in technology uptake. This is best accomplished in stages, at the pace appropriate for each site (Berg M. , 1999). EHR data completeness, accuracy, timeliness or currency of the data, and 
granularity are impacted by systems issues (Chan, Fowles, \&Weiner, 2010). This aligns with guidance from Stage Theory of Organizational Change, highlighting the stages of uptake, and diffusion of innovations surrounding health information technology - describing the social process by which systems are communicated and ultimately accepted or rejected. Taken as a whole, these theoretical frameworks provide a lens by which to examine the dynamics involved in EHR use and the ways in which challenges can be anticipated and overcome (California HealthCare Foundation, 2002).

\subsubsection{Testing external validity of falls risk identification models}

As noted in Aim 3 of this research, feasibility of conducting a test of external model validity was explored through a planned ROC analysis comparing patient risk identification using the gold standard Timed Get Up and Go test versus the EHR data-based risk identification model here explored (Hanley \& McNeil, 1983). A priori power analysis indicates need for 256 patients to have $80 \%$ power for detecting a medium sized effect using .05 criterion of statistical significance. While such an analysis is outside of the scope of the dissertation, future research using an appropriately sized data set could address external model validity.

\subsection{Conclusions}

This research supports a more in-depth understanding of issues vital to falls risk identification and ultimately prevention among older adults. In summary, increased public health efforts are needed to help foster a systems-based approach to falls risk identification and prevention in primary care. Primary care centers, especially in rural areas, often serve as the healthcare hub for communities. These centers are intimately linked with the care of their patients and are often already aware of community resources such as home health agencies, physical therapists, and other specialty care applicable to falls risks. However, the absence of 
established linkages and referral systems with these community resources contributes to unaddressed patient care needs. The complex health care needs of older adults combined with brief office visits in context of the absence of established communication and referral systems with local resources culminates in challenges which can be addressed only through enhancing the system of health care in these communities. We also find that falls risk factors are complex issues which are rarely addressed during routine care and inadequately documented in the EHRs. This obscures the public health problem of falls among older adults, and highlights the need for increased public health efforts to support practice facilitation and health systems improvement for falls risk identification, care for those risk factors, and ultimately prevention of falls. Fortunately in this research we find: 1) a clear want in developing a stronger sense of team-based care both within the clinic and across community-based partners; 2) viability in repurposing EHR data to build a data set of priority and extended falls risk factors for analysis; and 3) promise in being able to model falls risk using EHR data. Taken as a whole, these findings help to support the development of health systems better equipped to address falls among older adults. 


\title{
Appendix A: Aim 1 Institutional Review Board Approval
}

\author{
WestVirginiaUniversity. \\ Orlice of Research Integrity and Compliance

$\begin{array}{ll} & \text { Acknowledgement Letter Exempt Initial Protocol Review } \\ \text { To } & \text { Adam Baus } \\ \text { From } & \text { WVU Office of Research Integrity and Compliance } \\ \text { Approval Period } & 05 / 02 / 2014 \text { Expiration Date 05/01/2017 } \\ \text { Subject } & \text { Acknowledgement Letter Exempt Initial Protocol Review } \\ \text { Protocol Tracking } & \begin{array}{l}\text { 1403223131 } \\ \text { Title }\end{array} \\ & \begin{array}{l}\text { Understanding falls screening practices in select West Virginia primary care } \\ \text { centers }\end{array}\end{array}$

The above-referenced study was reviewed by the West Virginia University Institutional Review Board IRB and was granted exemption in accordance with 45 CFR 46.101 .

- This research study was granted an exemption because the Research involves educational tests, survey procedures, interview procedures or observation of public behavior and (1) information obtained is recorded in such a manner that human subjects cannot be identified, directly or through identifiers linked to the subjects; and (ii) any disclosure of the human subjects responses outside the research could not reasonably place the subjects at risk of criminal or civil liability or be damaging to the subjects financial standing, employability, or reputation [45 CFR 46.101(2)]. All exemptions are only good for three years. If this research extends more than three years beyond the approved date, then the researcher will have to request another exemption. The following documents have been acknowledged for use in this study and are available in the WVU+kc system:

- CONDITION OF APPROVAL: Once letters of permission are obtained from various sites, they must be submitted for approval with an amendment.

Documents for use in this study have been acknowledged and validated and are available in the WVUkc system in the Notes and Attachments section of your protocol.

If you have any questions, please contact the IRB at 3042937073.

Thank you.

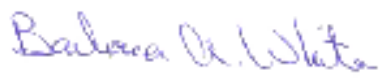




\title{
Appendix B: Focus Group Invitation Letter
}

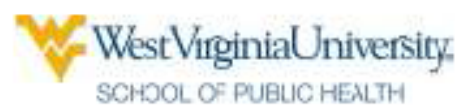

\author{
Administrator \\ Primary Care Center \\ Address \\ City, WV Zip
}

Date

Dear Administrator,

I invite the physicians, nurses, and medical staff of your organization to take part in a group discussion about falls screening practices in West Virginia primary care. This study is being conducted in partial fulfillment of my PhD in Public Health Sciences at West Virginia University. This study aims to understand the impeding and promoting determinants to falls screening, and the potential for electronic health record data-based clinical decision support to be incorporated into the care setting.

The group discussion would be led by me and Cecil Pollard with the WVU School of Public Health, Office of Health Services Research. The conversation will last about $\mathbf{4 5}$ minutes. This discussion would be held at your center, during lunch as to avoid disruption of patient services.

The following incentives are offered as an indication of our thanks and gratitude for participating:

- Copies of the Stopping Elderly Accidents, Deaths \& Injurles (STEADI) tool kit for health care providers from the Centers for Disease Control and Prevention

- Information on reimbursing insurers for falls risk assessments from the American Medical Association and the National Committee for Quality Assurance

- Information on meeting Physician Quality Reporting System and National Quality Forum guidelines for falls screening from the American Medical Association

- Lunch for all participants

The focus group would be audio-recorded in order to accurately record what is said. All information shared will remain confidential. Reports of study findings will not include any identifying information.

If you have any questions about this study, please contact me at 304-293-1083 or abaus@hsc.wvu.edu. This study has been approved by the West Virginia University Institutional Review Board (Protocol \#1 1403223131). We greatly value working with your organization.

Your signature on this form indicates agreement to participate. I will contact you after receiving this signed form to coordinate with you to schedule an appropriate time to hold the group discussion. You can return this form to me via email at abaus@hsc.wvu.edu or fax at 304-293-6685.

Adam Baus, MA, MPH

West Virginia University Office of Health Services Research

PhD Candidate, Public Health Sciences

Our organization agrees to participate.

Print Name, Title:

Signature:

Date:

SCHOOL OF PUELIC HENTH

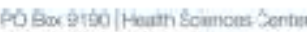

Morpentown WV 20502-9190

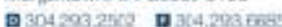




\section{Appendix C: Informed Consent Form}

\section{Understanding falls screening practices in select West Virginia primary care centers}

You are invited to participate in a group discussion about falls screening practices in primary care. This study is being conducted by Adam Baus with the West Virginia University School of Public Health in completion of the PhD in Public Health Sciences. You are invited to participate because you are part of the health center's care team. This study aims to understand the impeding and promoting determinants to falls screening, and the potential for electronic health record data-based clinical decision support to be incorporated into the care setting.

Participation is voluntary. If you agree to participate, you will take part in a group discussion with other members of the care team at your health center. The focus group will be led by Adam Baus and Cecil Pollard, both with the West Virginia University School of Public Health, Office of Health Services Research. The focus group will last about 45 minutes.

The focus group will be audio-recorded in order to accurately record what is said. You may request that the recording be paused at any time. You may also choose to leave the focus group at any time.

The information you will share with us will be kept confidential. Reports of study findings will not include any identifying information. Audio-recordings of the focus groups will be kept on a passwordprotected computer. The typed transcription will be kept on a password-protected computer and any printed copies will be kept in a locked file cabinet.

If you have any questions about this study, please contact Adam Baus at 304-293-1083 or abaus@hsc.wvu.edu.

Your signature on this consent form indicates agreement to participate in this study. You will be given a copy of this form to keep, whether or not you agree to participate. The second signed consent form will be kept by the researcher.

I have read the consent form and all of my questions about the study have been answered. I understand that the focus group will be audio recorded. I agree to participate.

Print name:

Signature:

Date: 


\section{Appendix D: Stopping Elderly Accidents, Deaths \& Injuries Toolkit (cover page only)}

\section{Algorithm for Fall Risk Assessment \& Interventions}

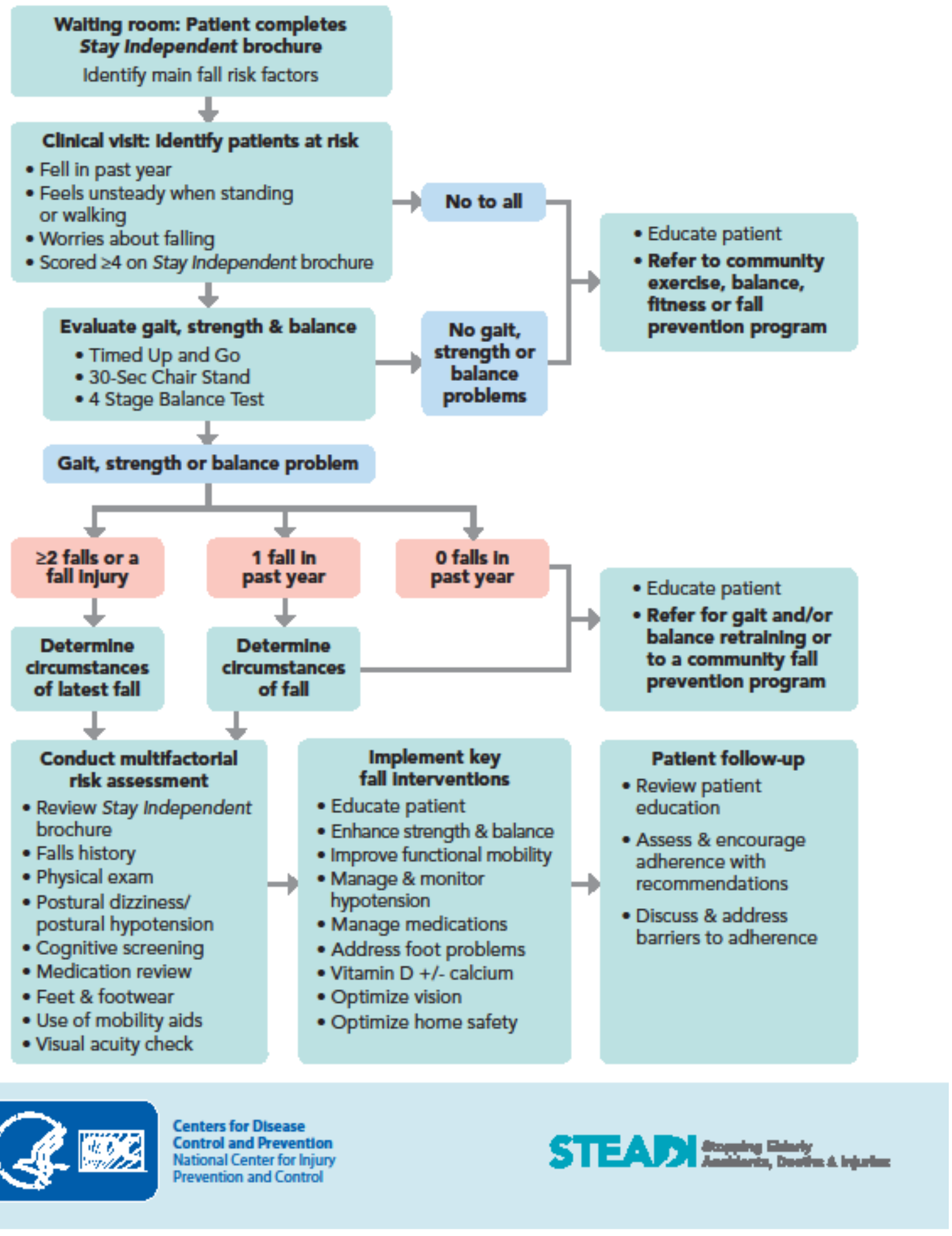




\section{Appendix E: Physician Quality Reporting System Measure 154 - Falls Risk Assessment}

"Measure \$154 (NQF: 0101): Falls: Risk Assessment

2013 PQRS OPTIONS FOR INDIVIDUAL MEASURES: CLAIMS, REGISTRY

This is a two-part measure which is paired with Measure $\$ 155$ : Falls: Plan of Care. If the falls risk assessment indicates the patient has documentation of two or more falls in the past year or any fall with injury in the past year (CPT II code 1100F is submitted), $\$ 155$ should also be reported.

\section{DESCRIPTION:}

Percentage of patients aged 65 years and older with a history of falls who had a risk assessment for falls completed within 12 months

\section{INSTRUCTIONS:}

This measure is to be reported a minimum of once per reporting period for patients seen during the reporting period. There is no diagnosis associated with this measure. This measure is appropriate for use in all non-acute settings (excludes emergency departments and acute care hospitals). This measure may be reported by cinicians who perform the quality actions described in the measure based on the services provided and the measure-specific denominator coding.

Measure Reporting via Claims:

CPT or HCPCS codes and patient demographics are used to identify patients who are included in the measure's denominator. CPT Category II codes are used to report the numerator of the measure.

When reporting the measure via claims, submit the listed CPT or HCPCS codes, and the appropriate CPT Category II codes OR the CPT Category II code(s) with the modfier. The modifiers allowed for this measure are: 1P- medical reasons, $8 \mathrm{P}$ - reason not otherwise specifed. All measure-specific coding should be reported on the claim(s) representing the eligible encounter.

Measure Reporting via Registry: CPT or HCPCS codes and patient demographics are used to identfy patients who are included in the measure's denominator. The numerator options as described in the quality-data codes are used to report the numerator of the measure.

The quality-data codes listed do not need to be submitted for registry-based submissions; however, these codes may be submitted for those registries that utilize cdaims data.

\section{DENOMINATOR:}

All patients aged 65 years and older who have a history of falls (history of falls is defined as 2 or more falls in the past year or any fall with injury in the past year)

Denominator Criteria (Eligible Cases):

Patients aged $\geq 65$ years on date of encounter

AND

Patient encounter during the reporting period (CPT or HCPCS): 97001, 97002, 97003, 97004, 99201 , 99202, 99203, 99204, 99205, 99212, 99213, 99214, 99215, 99304, 99305, 99306, 99307, 99308, 99309 , 99310, 99324, 99325, 99326 , 99327, $99328,99334,99335,99336,99337,99341,99342,99343,99344$, $99345,99347,99348,99349,99350$, G0402, G0438, G0439

Date: $11 / 16 / 2012$

Version 7.1

Page 325 of 637

CPT only copyright 2012 American Medical Associabion. All rights reserved. 
NUMERATOR:

Patients who had a risk assessment for falls completed within 12 months

Numerator Instructions: All components do not need to be completed during one patient visit, but should be documented in the medical record as having been performed within the past 12 months.

Definitions:

Fall - A sudden, unintentional change in position causing an individual to land at a lower level, on an object, the floor, or the ground, other than as a consequence of sudden onset of paralysis, epleptic seizure, or overwhelming external force.

Risk Assessment - Comprised of balance/gait AND one or more of the following: postural blood pressure, vision, home fall hazards, and documentation on whether medications are a contributing factor or not to falls within the past 12 months.

MUMERATOR NOTE: The correct combination of numerator code(s) must be reported on the claim form in arder to properly report this measure. The "correct combination" of codes may require the submission of mutiple numerator codes.

Numerator Quality-Data Coding Options for Reporting Satisfactorily:

Risk Assessment for Falls Completed

(Two CPT II codes B288F \& 1100F] are required on the claim form to submit this numerator option)

CPT II 3288F: Falls risk assessment documented

AND

CPT II 1100F: Patient screened for future fall risk, documentabion of two or more falls in the past year or any fall with injury in the past year

QR

Risk Assessment for Falls not Completed for Medical Reasons

(Two CPT II codes B288F-1P \& 1100F ] are required on the claim form to submit this numerator option) Append a modifier (IP) to CPT Category II code 3288F to report documented circumstances that appropriately exclude patients from the denominator.

3288F with 1P: Documentation of medical reason(s) for not completing a risk assessment for falls (i.e., reduced mobiity, bed ridden, immobile, confined to chair, wheelchair bound, dependent on

AND helper pushing wheelchair, independent in wheelchair or minimal help in wheelchair)

CPT II 1100F: Patient screened for future fall risk, documentation of two or more falls in the past year or any fall with injury in the past year

OR

If patient is not eligible for this measure because patient has documentation of no falls or anly one fall without injury the past year, report

Patient not at Risk for Falls

(One CPT II code [1101F] is required on the claim form to submit this numerator option)

CPT II 1101F: Patient screened for future fall risk; documentation of no falls in the past year or only one fal without injury in the past year

OR

If patient is not eligible for this measure because falls status is not documented, report: Falls Status not Documented

(One CPT II code [1101F-8P] is required on the claim form to submit this numerator option)

Date: $11 / 16 / 2012$

Version 7.1

CPT only copyright 2012 American Medical Associabion. All rights reserved.

Page 326 of 637 
Append a reporting modifer (8P) to CPT Category II code $1101 \mathrm{~F}$ to report circumstances when the patient is not eligible for the measure.

$1101 \mathrm{~F}$ with 8P: No documentation of falls status

$\underline{\mathrm{OR}}$

Risk Assessment for Falls not Completed, Reason not Otherwise Specified

(Two CPT II codes B288F -8P \& 1100F] are required on the daim form to submit this numerator option)

Append a reporting modifer (8P) to CPT Category II code $3288 \mathrm{~F}$ to report circumstances when the action

described in the numerator is not performed and the reason is not otherwise specified.

$3288 \mathrm{~F}$ with 8P: Falls risk assessment not completed, reason not otherwise specified

AND

CPT II 1100F: Patient screened for future fall risk, documentation of two or more falls in the past year or any fall with injury in the past year

\section{RATIONALE:}

Screening for specific medical condifions may direct the therapy. Although the clinical guidelines and supporting evidence calls for an evaluation of many factors, it was felt that for the purposes of measuring performance and faciitating implementation this inifial measure must be limited in scope. For this reason, the work group defined an evaluation of balance and gait as a core component that must be completed on all patients with a history of falls as well as four additional evaluations - at least one of which must be completed within the 12 month period. Data elements required for the measure can be captured and the measure is actionable by the physician.

\section{CLINICAL RECOMMENDATION STATEMENTS:}

Older people who present for medical attention because of a fall, or report recurrent falls in the past year, or demonstrate abnormalities of gait and/or balance should be offered a multifactorial falls risk assessment. This assessment should be performed by a health care professional with appropriate skils and experience, normally in the setting of a specialist falls service. This assessment should be part of an individualized, multifactorial intervention. (NICE) (Grade C)

Multifactorial assessment may include the following:

- identification of falls history

- assessment of gait, balance and mobility, and muscle weakness

- assessment of osteoporosis risk

- assessment of the older person's perceived functional ability and fear relating to falling

- assessment of visual impairment

- assessment of cognitive impaiment and neurological examination

- assessment of urinary incontinence

- assessment of home hazards

- cardiovascular examination and medication review (NICE) (Grade C)

A falls risk assessment should be performed for older persons who present for medical attention because of a fall, report recurrent falls in the past year, report dfficulties in walking or balance or fear of falling, or demonstrate unsteadiness or difficulty performing a gait and balance test.

The falls risk evaluation should be performed by a clinician with appropriate skills and experience. [C]

A falls risk assessment is a clinical evaluation that should include the following, but are not limited to:

- a history of fall circumstances

- review of all medications and doses

- evaluation of gait and balance, mobility levels and lower extremity joint function

- examination of vision

Date: $11 / 16 / 2012$

Version 7.1

CPT only copyright 2012 American Medical Association. All rights reserved. 
- examination of neurological function, muscle strength, proprioception, reflexes, and tests of cortical, extrapyramidal, and cerebellar function

- cognifive evaluation

- screening for depression

- assessment of postural blood pressure

- assessment of heart rate and rhythm

- assessment of heart rate and rhythm, and blood pressure responses to carotid sinus stimulation if appropriate

- assessment of home environment

The falls risks assessment should be followed by direct intervention on the identified risk. [A] (AGS)

Date: $11 / 16 / 2012$

Version 7.1

CPT only copyright 2012 American Medical Associafion. All rights reserved.

Page 328 of 637 


\section{Appendix F: Qualitative Analysis Codebook}

Stage 1 coding

\section{Conversation area}

Experiences in caring for older adults

\section{Coding}

Coding description

Complex care needs

This node includes all references to complexities in caring for older adult patients.

(1)
complicated when you're trying to deal with things, because one illness, you try to do something that might effect perhaps the other illnesses.

Reference 2. Focus Group 4: "Yeah, that's true, and also, not only is the resource not there, but $* * *$ brings up the point that there's a lot of different factors. Falls is a very complex problem. So it's also a Pandora's Box that, if you're already dealing with a lot of other medical problems, you might feel like, "I don't have the time to try and figure out what's causing their falls."

Difficulty communicating to patients

Generational differences
This node includes all references to difficulties in communicating with older adult patients. Reference 1. Focus Group 3: "You don't know what they understand."

Reference 2. Focus Group 4: 'They can't hear, and you're yelling. I mean really, you're yelling. And you hope that you're getting your message across."

This node includes all Reference 1. Focus Group 2: references to perceived "Sometimes, it's a whole generational differences among different experience. They older patients (i.e., patients $65+$ ) have a whole different set of lifestyles and ways of thinking about the Great compared to younger 
Stage 1 coding

Conversation area

Coding

Coding description

patients.

get rid of anything."

Reference 2. Focus Group 2:

'I think they're better about keeping appointments than the younger generation. If they have an appointment, they're going to come to their appointment. They're gonna come to their bloodwork. I mean, we do reminder calls. I think as far as that, I think they're more responsible with their own time."

Informal ways of This node includes all identifying falls risk references to informal ways in which participants reported identifying indicators of falls risks.
Lacking knowledge This node includes all about patient circumstances
Reference 1. Focus Group 1:

"Actually, your movement of a patient from the waiting room back to the exam room, the nurse, MA, whoever is with them is going to know if they have a mobility problem. True?"

Reference 2. Focus Group 3: 'I don't think there's a formal screening that I use per se, but I watch them as they get up from their chair and walk over to the exam room. I usually watch them walking down the hallway, if they're ambulating at all. And of course just questions." Reference 1. Focus Group 3: "I find it frustrating. You don't know what they understand, you don't know what they're doing at home. You don't know if they're taking their medication. You don't know if they're taking their medication correctly. 
You don't know if they're falling until you get information from the hospital that says, "Patient fell and cracked their head open." You just don't know, unless they've got..."

Reference 2. Focus Group 4: "and when it comes to falls, the first thing I'm thinking is someone to help figure out what's going on at home."

Need for home safety

Patient independence
This node includes all references to participants noting the need for home safety to help prevent falls.
Reference 1. Focus Group 2:

"I mean, we all know the dangers of, kind of throw rugs in their home, but those of us that have never done home health or made home visits, we can tell them these things. Until they fall and break that hip, *** will tell you, unless you have a caregiver who takes up those rugs and declutters the place, you might as well howl at the moon, because that's all it is."

Reference 2. Focus Group 4: "And we try to make sure they have all the safety equipment they need for home."

This node includes all Reference 1. Focus Group 1: references to the "Certainly, issues of giving up independence to one's children. They raised you and now you're putting restrictions on them as they fail."

Reference 2. Focus Group 2. 
Stage 1 coding

$\begin{array}{llll}\text { Conversation area } & \text { Coding } & \text { Coding description } & \text { Examples }\end{array}$

"And I think it all comes

back to that independence.

Or they don't want to bother

anyone. It's their routine.

You wash your windows in

the spring and you wash

them in the fall."

Patient reluctance to This node includes all Reference 1. Focus Group 2:

use assistive or references to

"I'd probably fail that test. I

safety devices

reluctance among would. I probably would. I

patients to use assistive mean, by the time I got up, devices, such as canes, 10 seconds would be up. walkers, wheelchairs, [laughter] I've had my share bathtub hand rails, etc. of falls, and it's very embarrassing. But it's not embarrassing enough to use that cane all the time."

Reference 2. Focus Group 3:

"A lot of times they are very resistant to getting help, such as a walker or a cane. Or bringing someone into the house to help them."

Patient transportation problems

Rewarding to care for older patients
This node includes all references to patients experiencing difficulty in transportation needed for activities of daily living.

Reference 1. Focus Group 2:

"I have a woman, she does not leave her house unless it's for a doctor's appointment. She says, 'You just tell me and I'll do my blood work and my visit all in one day." And she said, 'You can call me, but I do not leave my house.' I was like, 'Okay.'”

Reference 2. Focus Group 4: "We have an issue with transportation, when it comes to caring for them."

This node includes all Reference 1. Focus Group 3: references to 'I think I agree with ***, it's participants reporting frustrating, but it's also very 
Stage 1 coding

Conversation area

Coding

Coding description

Examples

finding it rewarding to rewarding to take care of

Treating older adults differently
Falls among older
Falls triggering help care for older adult patients.
This node includes all references to older adults being treated differently in society compared to younger individuals. older people, especially those who don't have a lot of resources and ability to care for themselves, and family support, because you're really their only support they have in some situations."

Reference 2. Focus Group 4: Respondent A: "We had a lot of time. It [frail elder program] had a lot of impact. I really enjoyed doing it. It's probably been a year and a half, two years since I had that. I'm not sure."

Respondent B: "They still call for her services."

Reference 1. Focus Group 2: Respondent A: "When you have a cane, people can ignore you instead of talking to you. It's just like if you're in a wheelchair. People talk to whoever's with you." Respondent B: "Or a walker. And we do the same thing. If we have somebody that comes in here with a walker, we tend to talk to whoever's with them. We all do it."

Reference 2. Focus Group 3: "There's a level of disrespect when it comes to being older. People don't have the drive to care for their elderly family members as they once did, and they still do in other cultures."

This node includes all Reference 1. Focus Group 1: 
Stage 1 coding

\begin{tabular}{|c|c|c|c|}
\hline Conversation area & Coding & Coding description & Examples \\
\hline \multirow[t]{3}{*}{ adults } & decline & $\begin{array}{l}\text { references to falls } \\
\text { among older patients } \\
\text { triggering subsequent } \\
\text { health events. }\end{array}$ & $\begin{array}{l}\text { "That's probably the first } \\
\text { thing that puts them into the } \\
\text { home of someone else, or } \\
\text { someone else in their home, } \\
\text { I'm guessing." }\end{array}$ \\
\hline & & & $\begin{array}{l}\text { Reference } 2 \text {. Focus Group } 2 \text { : } \\
\text { "And my father was in a } \\
\text { dementia facility. He just } \\
\text { went there in May. And he } \\
\text { had several falls, and he fell } \\
\text { in July and hit his head, and } \\
\text { it killed him. And so it's a } \\
\text { huge thing. And my mother } \\
\text { in law died of a head injury } \\
\text { too, from a fall in the } \\
\text { bathroom. On the death } \\
\text { certificate, it doesn't say that. } \\
\text { It says, "congestive heart } \\
\text { failure." It says, "COPD" on } \\
\text { my mother in law's, but } \\
\text { that's not what killed her. So } \\
\text { I think there's a whole lot } \\
\text { more falls that we don't } \\
\text { know about. Those are } \\
\text { anecdotal stories, but I think } \\
\text { there's a whole lot of people } \\
\text { that fall is their demise. And } \\
\text { I don't know the answer. I } \\
\text { wish I did." }\end{array}$ \\
\hline & $\begin{array}{l}\text { Falls underreported } \\
\text { by patients }\end{array}$ & $\begin{array}{l}\text { This node includes all } \\
\text { references to falls } \\
\text { being underreported by } \\
\text { patients. }\end{array}$ & $\begin{array}{l}\text { Reference } 1 . \text { Focus Group } 2 \text { : } \\
\text { "What was what's her name, } \\
\text { that we had to help hold her } \\
\text { as she came to the lab } \\
\text { yesterday? What was her } \\
\text { name? But she had this big } \\
\text { bruise on her arm, and I said, } \\
\text { "Oh, did you hit yourself?"" } \\
\text { And she said, "No, but I fell } \\
\text { and cracked my head off the } \\
\text { cement." And I was like, } \\
\text { 'Oh."' }\end{array}$ \\
\hline
\end{tabular}


Stage 1 coding

Examples

Reference 2. Focus Group 3:

'No. I think it's commoner

than we know, and I think

anyone that's older - and

there was an article in the

New York Times, of course

it was about an assisted

living community — but

people hide it. They don't

want anyone to know,

because they're afraid if they fall, "Somebody's going to

put me into a care facility, somebody's going to put me into an upper level care facility, or that's going to be more expensive."'"

Role of primary care is in falls prevention among older adults
Absence of policies and procedures

This node includes all references to absence of health center policies and procedures in addressing accidental falls among older adults.
Difficulty in addressing falls
Reference 1. Focus Group 1: 'I don't think it happens all the time. Like automatically checking an alc in a diabetic. I don't think it's a reflex, "We need to do it," type of thing yet. I don't think that the awareness is all that great yet, and I don't think we comply with doing that every single visit also."

Reference 2. Focus Group 4: "But is there something more that we should be doing?

Like should more than eight meds on their list trigger an in-depth, maybe polypharmacy? Should something else trigger it? Or should we just leave each individual clinician to use their magical medical powers?"

This node includes all Reference 1. Focus Group 2: references to
"Yeah, especially, was it last 
Stage 1 coding

\begin{tabular}{|c|c|c|c|}
\hline Conversation area & Coding & Coding description & Examples \\
\hline & factors & $\begin{array}{l}\text { participants expressing } \\
\text { difficulty in addressing } \\
\text { risk factors associated } \\
\text { with accidental falls } \\
\text { among older adults. }\end{array}$ & $\begin{array}{l}\text { week when I worked with } \\
* * * \text { ? That last patient of the } \\
\text { morning, that he had been } \\
\text { dizzy and fatigued? Oh, that } \\
\text { was a huge workup for him. } \\
\text { He's coming back. We did } \\
\text { some tests and some labs, } \\
\text { and that kind of stuff, and } \\
\text { he's coming back. And you } \\
\text { can spend thousands of } \\
\text { dollars on a dizziness } \\
\text { workup and send them to } \\
\text { neurology, and send them } \\
\text { here and there, and not get an } \\
\text { answer." }\end{array}$ \\
\hline & & & $\begin{array}{l}\text { Reference } 2 \text {. Focus Group } 4 \text { : } \\
\text { "I don't have the time to try } \\
\text { and figure out what's causing } \\
\text { their falls. That's the barrier, } \\
\text { because falls is a very } \\
\text { complex - there's a lot of } \\
\text { things you have to think } \\
\text { about and figure out." }\end{array}$ \\
\hline & $\begin{array}{l}\text { Educating patients } \\
\text { on falls risks }\end{array}$ & $\begin{array}{l}\text { This node includes all } \\
\text { references to } \\
\text { respondents expressing } \\
\text { their role in educating } \\
\text { patients on falls risk } \\
\text { factors. }\end{array}$ & $\begin{array}{l}\text { Reference } 1 \text {. Focus Group } 3 \text { : } \\
\text { "So we get off into other } \\
\text { risks for falls too. And then I } \\
\text { think about these patient } \\
\text { summaries, where you } \\
\text { include that education in } \\
\text { something you give the } \\
\text { person to take home. So I'll } \\
\text { always put that into patient } \\
\text { education, the first thing in } \\
\text { the summary, little things to } \\
\text { prevent falls." }\end{array}$ \\
\hline & & & $\begin{array}{l}\text { Reference } 2 \text {. Focus Group } 4 \text { : } \\
\text { "September I think is fall } \\
\text { month or something like that. } \\
\text { I would send out postcards } \\
\text { with little tips on fall } \\
\text { prevention, and we also gave }\end{array}$ \\
\hline
\end{tabular}


Stage 1 coding

Examples

them nightlights, because a lot of them would get up at night and go to the bathroom with no lights on anywhere, and they would fall. So we'd get nightlights. And also gave them like the socks that you get in the hospital that have the grips. We gave out those, and different things."

Financial barriers to This node includes all falls screening

references to respondents expressing perceived financial barriers to falls screening in primary care.
Reference 1. Focus Group 1:

"We have a different reimbursement structure. So I don't care if you do $\$ 300$ worth of stuff on a Medicare patient; you're only going to get $\$ 100$. It doesn't matter. Same with Medicaid. You give them all their immunizations, do their health maintenance exam and you only get $\$ 111$, period. If they come in for a cold, you get $\$ 111$. So it doesn't matter what you do; you get $\$ 111$. It may settle up in two years, and then we'll get $\$ 115$. [laughter] But you've expanded that much extra money for the two years until you've demonstrated that you've spent more than $\$ 111$, on average."

Reference 2. Focus Group 3: "You could automatically say, 'Anybody who's over this age, we're going to go ahead and give them fall screening.' That would be easy enough. But the problem is who's going to 
Examples

give them the information?

Who's going to pay for them to give the information? Ten minutes on patients in the morning, that's a lot of time. Who's going to pay for that time?"

Ideas spurred on practice changes

Need for home health
This node includes all references to instances in which ideas on practice changes were spurred by the focus group discussion.
Reference 1. Focus Group 1:

Respondent A: "Hey ***, if they're wanting the nurses to do that, is that something that could be added into social history?"

Respondent B: "That's a good place for it."

Respondent A: "Can it be like history of falls? And then you could document in the box."

Reference 2. Focus Group 2: "I'm going to be honest with you, until we had this - if somebody comes in and it's obvious they're falling, I'll say, 'Oh, gosh, let's write you for a quad cane, whatever." That's pretty much as far as it goes. I haven't referred a lot for gait training, to PT or anything. And if I do, are they gonna go? I should do the referrals, and at least I've done everything I can, but..."

This node includes all references to Reference 1. Focus Group 1: "As a matter of fact, I have a patient who told me that they were going to Amish country in a bus with all these older folks, and they have a blast. [laughter] So they do have some neat programs like that 
Stage 1 coding

Conversation area

Coding Coding description

for aging. But home care

locally also goes in home to

assess safety in-house, to see

if they need any kind of

safety equipment to help

them get out, sit down and

get up from the commode,

transfer easy like back and

forth from the bathtub, if

they can't shower."

Reference 2. Focus Group 2:

"Also, when we use house

calls, home health, and that's

part of their initial

assessment when they go

into the home, is safety and

fall risk in the home. So

they'll do that, and they'll

call us and say, "I think they

could use a raised toilet seat

or some grab bars in the

shower." And we can call

Life Guard Medical and

they'll go out and install the

stuff. We just send the order

to them with the patient's

demographics, and then they

Need for teambased care
This node includes all

references to

respondents stating the

value of having

interdisciplinary care

team members

involved in falls care

among older adults. go into the home."

Reference 1. Focus Group 3:

“**** brought up another

possibility, which you

mentioned PT. Never done

it, but I send plenty of older

people to PT when they need

more consistent, regular

treatments for their arthritis

or needs. I guess it's

possible you could ask the

physical therapist to do a fall

risk assessment. Because

they're going to be watching

them on a treadmill or on a 
$\begin{array}{llll}\text { Conversation area } & \text { Coding } & \text { Coding description } & \text { Examples }\end{array}$

stationary bike or doing any strength tests, working with them individually, will observe them for longer periods of time with activities. I don't know that they can do that; does anybody? "

Reference 2. Focus Group 4: "Yeah, a provider would say... They started the program, then I'd go to 'em and ask 'em if they had someone that was at high risk for fall, to let me evaluate them, and then I also got to do the home visits. So I could have up to 50 patients I'd do home visits on three times a year. I did that for quite a while."

Reluctance to screen This node includes all Reference 1. Focus Group 1: references to "I get a lot of this stuff respondents expressing comes across from insurance reluctance to screen for companies, Medicare and falls risk factors among Blue Cross/Blue Shield. older adults.

Quality nurse comes and talks about a bunch of things, wants everybody screened for falls. And I have $20 \%$ of my A1cs greater than nine. We can only deal with so many things. And I'm not going to throw not one more thing on to the providers, because they're already busy enough."

Reference 2. Focus Group 4: Respondent A: "So if they said, "We've found good 

falls and decreased nursing home admissions and decreased whatever..." Respondent B: "Decreased dollars."

Respondent A: "Yeah. Decreased hospital admissions - then I would be more likely to want to do it, rather than just it seems like a good idea, which it does."

Visits not dedicated This node includes all to falls prevention references to office Reference 1. Focus Group 1: visits among older patients not solely addressing falls risk. "So yes, if the patient is smoking, I like to do a smoking cessation talk every time. I like to do a cage question every time I see a drunk. I like to do intimate partner violence every time I see a person who has that there. So all these activists and their little thingies, the screenings, I like to do those, but if I've got 15 minutes to see a chronic, complicated patient, plus I've got to walk in there or work in there with some other problem, and plus my computer is not cooperating, that time is gone. I can't do it. I like to do fall precautions and fall preventions and refer them to this and that, but ..."

Reference 2. Focus Group 2: Respondent: 'It depends on the patient and what all we have to do too. If somebody's just come in for 


\section{$\begin{array}{llll}\text { Conversation area } & \text { Coding } & \text { Coding description }\end{array}$}

a blood pressure, then it's not as much as if they're coming in for diabetes and blood pressure and cholesterol and 500 other things."

Potential for using Inconsistent use of your EHR to the EHR

manage and use that data for falls screening
Yes potential
This node includes all references to inconsistent use of electronic health records for entry and management of falls risk information.
Reference 1. Focus Group 1: "And a lot of it isn't charted. The other people see it, though. And what you might think is irrelevant might not be irrelevant to somebody else. So if you didn't chart it, then somebody else didn't see it, and they think, "Well, that person's okay," so it depends on what you think is relevant versus their information as well."

Reference 2. Focus Group 4: "Oh, this person falls." But just one fall, I wouldn't put it on the problem list, and I would be willing to bet that the EHR probably does have some checkbox for falls, because fall prevention is one of those things that... [laughter]"

This node includes all references to participants indicating the potential for electronic health records to be a viable tool for falls screening among older adults.
Reference 1. Focus Group 1: 'I'm sitting here thinking about what you said about using our EMR and having some sort of flag or popup. As for myself, it would be nice. If I'm taking someone back to my X-ray department, which is all the way on the other end of the building, I would like to know that they are at risk for a fall, because I'm walking them down this hallway by 
Stage 1 coding

$\begin{array}{llll}\text { Conversation area } & \text { Coding } & \text { Coding description } & \text { Examples }\end{array}$

myself. You know what I mean? And I guess it would be nice maybe to have a little popup or even walking them back to the lab, even for the front office staff to say, okay, this patient's at risk for a fall. I know they're just walking right to there, but maybe I should walk with them for just a second. So I'm sitting here thinking it would be nice. I understand it would be kind of time consuming to put everything in and to do an assessment, but maybe for some of us..."

Reference 2. Focus Group 3: "I'd like to reiterate that the EHR patient summary is a big thing for patient education. That really does improve that communication. Elderly people especially don't remember that education offered in the office. You can't count on them knowing what you told them five minutes later. So having it in writing right after the visit, if you can be that efficient, clearly is a big addition to patient education."

Thoughts on using EHR data to identify at-risk patients
Organizational barriers to EHR use

This node includes all references to health center organizational barriers preventing use of electronic health records for identification of older patients at-risk for
Reference 1. Focus Group 1: 'I think there's also a human element to this too - how best to get the providers to then pick up on that information and follow through on it? There's a little bit of a behavioral part to 
Stage 1 coding

falls.

Technology barriers to EHR use
This node includes all references technological barriers to using electronic health records for identification of older patients at-risk for falls. that. And it's not just the EHR, but there's also the... [laughter]'"

Reference 2. Focus Group 3: "It would be helpful if that's exactly what happened - is if you walked into [laughter] and the score was there, and you just had to deal with it. That would be helpful. But having to have a lot of people enter data to get the score would not be especially helpful. Our nurses can't enter a lot of data, because they're busy as well."

Reference 1. Focus Group 3:

"It needs to be on the template because there's a whole bunch of check-off stuff we have that's on what I call the chart page, and that would mean going out of the note. Then you've got a computer delay. Every time you forget to do something or you have to go back and edit a note, you've got a computer delay and you forgot to do something, and then by the time the page flashes up, maybe you forgot what you were doing, because you've got Alzheimer's.

Reference 2. Focus Group 4: 'I bet there's something in the EHR, but the problem is there's so many things to check off and ask in the intake that you can get - c 


\title{
Appendix G: Aims 2 and 3 Institutional Review Board Approval
}

\author{
WestVriginiaUniversity. \\ Oflice of Research Integrity and Compliance

\section{Acknowledgement Letter Not Human Subject Research NHSR} \\ To \\ From \\ Approval Period \\ Subject \\ Protocol Tracking \\ Title \\ Adam Baus \\ WVU Office of Research Integrity and Compliance \\ 03/19/2014 Expiration Date 03/18/2019 \\ Not Human Subject Research Ackmowledgment \\ 1402217616 \\ Using secondary, de-identified electronic health record data to identify cases of \\ older patients at risk for falls
}

Thank you for your submission to the West Virginia University Institutional Review Board IRB.

It has been determined that your project does not meet the definition of human subject research for the following reasons

- In order to be considered human subject research, individually identifiable private information must be obtained or used in the research. If there is no individually identifiable private information involved, the project is not human subject research and does not require being submitted to the Office of Research Integrity \& Compliance. Private information must be individually identifiable (ie., the identity of the subject is or may be readily ascertained by the investigator or someone else associated with the information) in order to constitute research involving human subjects.

If you have any questions, please contact the IRB at 3042937073.

Thank you.

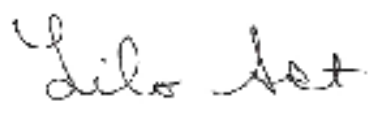

Board Designee Lilo Ast

Letter Sent By Lilo Ast on 03/19/2014 at 17:53:33-04:00 


\section{Appendix H: Priority Falls Risk Factors, Locations of Data Extraction, and Coding}

\begin{tabular}{|c|c|c|}
\hline Factor & Location & Coding \\
\hline Age $>=65$ & Demographics & Age categories calculated from date of birth \\
\hline \multirow[t]{2}{*}{$\begin{array}{l}\text { Cognitive } \\
\text { impairment }\end{array}$} & ICD-9 codes & $\begin{array}{l}\text { Cognitive disorder } 294.1 \text { - 294.9; Senile dementia } 290.0 \text { - } \\
\text { 290.3; Vascular dementia 290.4; Dementia with Parkinson's } \\
\text { disease 331.82; Mild cognitive impairment } 331.83\end{array}$ \\
\hline & $\begin{array}{l}\text { Medcin } \\
\text { findings }\end{array}$ & $\begin{array}{l}\text { AGE-RELATED COGNITIVE DECLINE 312268.00; } \\
\text { COGNITIVE DISORDER 312247.00; Cognitive Functions } \\
\text { Current Level Impaired 203821.00; Cognitive Functions } \\
\text { Current Level Impaired Mild 297368.00; Cognitive } \\
\text { Functions Current Level Superior 203819.00; Cognitive } \\
\text { Functions Current Level Totally Dependent 242551.00; } \\
\text { Cognitive Functions Decreased 203809.00; Cognitive } \\
\text { Functions Decreased From Premorbid Estimate 203810.00; } \\
\text { Cognitive Mini-Mental Status Exam Abnormal 296520.00; } \\
\text { LATE CVD EFFECTS - COGNITIVE DEFICITS } \\
\text { 98682.00; MILD COGNITIVE IMPAIRMENT 335113.00; } \\
\text { No Cognitive Function 8369.00; URINARY } \\
\text { INCONTINENCE DUE TO COGNITIVE IMPAIRMENT } \\
\text { 313474.00; DEMENTIA 272570.00; DEMENTIA } \\
\text { KNOWN (AXIS III) ETIOLOGY WITH BEHAVIOR } \\
\text { DISTURBANCE 214080.00; DEMENTIA OF } \\
\text { ALZHEIMER'S TYPE 278232.00; DEMENTIA OF } \\
\text { ALZHEIMER'S TYPE WITH BEHAVIOR } \\
\text { DISTURBANCE 278234.00; DEMENTIA OF } \\
\text { ALZHEIMER'S TYPE WITH EARLY ONSET 312241.00; } \\
\text { DEMENTIA OF ALZHEIMER'S TYPE WITH LATE } \\
\text { ONSET 312242.00; DEMENTIA OF KNOWN (AXIS III) } \\
\text { ETIOLOGY 35732.00; DEMENTIA OF UNKNOWN } \\
\text { (AXIS III) ETIOLOGY 35733.00; DEMENTIA OF } \\
\text { UNKNOWN (AXIS III) ETIOLOGY WITHOUT } \\
\text { BEHAVIORAL DISTURBANCE 314928.00; DEMENTIA } \\
\text { WITH BEHAVIORAL DISTURBANCE 318503.00; } \\
\text { DEMENTIA WITH LEWY BODIES 272878.00; } \\
\text { DEMENTIA, PATCHY 350856.00; FRONTOTEMPORAL } \\
\text { DEMENTIA 272877.00; HEAD INJURY WITH } \\
\text { DEMENTIA WITHOUT BEHAVIORAL DISTURBANCE } \\
\text { 312231.00; PARKINSON DISEASE W/ DEMENTIA W/O } \\
\text { BEHAVIORAL DISTURBANCE 312237.00; } \\
\text { PARKINSON DISEASE WITH DEMENTIA 38397.00; } \\
\text { PRESENILE DEMENTIA 312345.00; PRESENILE }\end{array}$ \\
\hline
\end{tabular}


DEMENTIA UNCOMPLICATED 312420.00; PRESENILE DEMENTIA WITH DELIRIUM 312421.00; PRESENILE DEMENTIA WITH DEPRESSED MOOD 312423.00; SENILE DEMENTIA 312559.00; SENILE DEMENTIA WITH DELUSIONAL FEATURES 312561.00; VASCULAR DEMENTIA 32694.00; VASCULAR DEMENTIA UNCOMPLICATED 38381.00; VASCULAR DEMENTIA WITH DELUSIONS 38383.00; VASCULAR DEMENTIA WITH DEPRESSED MOOD 38384.00

General notes Like "*Cognitive*" Or Like "*Dementia*" And Not Like "*flexibility*" And Not Like "*anxiety management*" And Not Like "*therapy*" And Not Like "*education*" And Not Like "*average*" And Not Like "*guided practice*" And Not Like "*normal*"

Dizziness- $\quad$ ICD-9 codes Vertigo

Medcin findings

Dizziness and giddiness, Light-headedness, Vertigo NOS 780.4; Vertigo 438.85

anxiety with dizziness or unsteady feelings 1179.00; BENIGN PAROXYSMAL POSITIONAL VERTIGO 32046.00; BENIGN PAROXYSMAL POSITIONAL VERTIGO BOTH EARS 312213.00; BENIGN PAROXYSMAL POSITIONAL VERTIGO LEFT EAR 312212.00; BENIGN PAROXYSMAL POSITIONAL VERTIGO RIGHT EAR 312211.00; BENIGN PAROXYSMAL VERTIGO OF CHILDHOOD 95303.00; CHLAMYDIAL INFECTIONS EPIDEMIC VERTIGO 97497.00; dizziness 650.00; dizziness episodes are recurrent 654.00; dizziness preceded 281450.00; dizziness preceded by chest pain 281455.00 ; dizziness preceded by flushing 281460.00; dizziness preceded by nausea 281461.00; dizziness preceded by sudden or severe headache 281451.00; dizziness preceded by sweating 281459.00; dizziness upon bending over 652.00; dizziness upon rolling over 2099.00; dizziness upon standing up 653.00; dizziness upon turning the head 116398.00; dizziness when walking up stairs 2100.00; dizziness while using hands or arms 651.00; headache preceded by everything spinning around (vertigo) 74.00; LATE CVD EFFECTS - VERTIGO 272323.00; PERIPHERAL VERTIGO 98368.00; spinning dizziness (vertigo) 655.00; spinning dizziness after rolling over 282960.00; spinning dizziness after turning the head 282961.00; spinning dizziness caused by noise 2009.00; spinning dizziness upon lying down 656.00; spinning dizziness with sudden changes in position 657.00; VERTIGO 275474.00; VERTIGO 
AURAL 275475.00; VERTIGO OF CENTRAL ORIGIN 96984.00; VERTIGO OF CENTRAL ORIGIN WITH MALIGNANT POSITIONAL VERTIGO 275478.00; VERTIGO OF CENTRAL ORIGIN WITH POSITIONAL NYSTAGMUS 275477.00; VERTIGO OTOGENIC 275476.00

General notes Like "*Dizzi*" Or Like "*Dizzy*" Or Like "*Vertigo*"

Fear of General notes Like "fall" And Like "*fear*" Or Like "*afraid*" Or Like falling "*worr*" Or Like "*scare*" Or Like "*fright*" Or Like "*concern*"

Gait-Balance ICD-9 codes impairment

Medcin findings

Abnormality of gait 781.2; Difficulty in walking 719.7; Lack of coordination 781.3; Other musculo skeletal symptoms referable to limbs 729.89

ATAXIC GAIT 278528.00; Ataxic Gait - Staggering Or Falling To The Right 9038.00; Balance Limited While Shifting Weight 208797.00; difficulty with balance 743.00; DISTURBANCE OF GAIT 278527.00; Gait - Antalgic 66733.00; Gait - Ataxic 9037.00; Gait - Ataxic, WideBased 9040.00; Gait - Hemiparetic 11143.00; Gait Hemiparetic, Left Side 11145.00; Gait - Hemiparetic, Right Side 11144.00; Gait - Insufficient For Exercise Testing 155110.00; Gait - Limping 9036.00; Gait - Scissoring 262002.00; Gait - Shuffling 9800.00; Gait - Spastic 9043.00; Gait - Spastic, Right-Sided 9044.00; Gait Stooped 240147.00; Gait - Swing Phase Foot Drop Left 8095.00; Gait - Swing Phase Foot Drop Right 8094.00; Gait - Toe Walk 11875.00; Gait - Waddling (Trendelenburg) 9048.00; Limited Balance 132533.00; PARALYTIC GAIT 278529.00; Sensation Romberg's Sign (balance lost without visual clues) 8909.00; SPASTIC GAIT 278530.00; STAGGERING GAIT 278531.00; Tandem Gait Test OffBalance To Left 261242.00; waddling gait 736.00

General notes Like "*Balance*" Or Like "Gait*" And Not Like "*Electrolyte*" And Not Like "*Denies*" And Not Like "*Meal*" And Not Like "*Outstanding*" And Not Like "*previous*" And Not Like "*revious*" And Not Like "*Food*" And Not Like "*Chemical*"

Gender Demographics Patient gender: F Female; M Male

Hearing ICD-9 codes Hearing loss $389.0-389.9$ impairment

Medcin

CENTRAL HEARING LOSS 37605.00; CONDUCTIVE 
HEARING LOSS 34074.00; CONDUCTIVE HEARING LOSS BOTH EARS 312207.00; CONDUCTIVE HEARING LOSS LEFT EAR 312206.00; CONDUCTIVE HEARING LOSS RIGHT EAR 312205.00; CONDUCTIVE HEARING LOSS, TYMPANIC MEMBRANE 37599.00; CONGENITAL EAR DEFORMITY CAUSING IMPAIRMENT OF HEARING 211210.00; difficulty hearing over background noise 282644.00; Hearing Difficulties 1002433.00; HEARING LOSS 34076.00; Hearing Loss 6676.00; Hearing Loss Bilaterally 6677.00; Hearing Loss Bilaterally Total 9445.00; Hearing Loss Left Only 6679.00; hearing loss left side only 145.00; Hearing Loss Right Only 6678.00; hearing loss right side only 144.00; Hearing Reception Threshold Whispered Voice Not Heard 155103.00; Hearing Services Hearing Aid Currently Being Worn 4055.00; loss of hearing 141.00; loss of hearing fluctuates 111726.00; loss of hearing for a month or more 1620.00; loss of hearing getting progressively worse 142.00; loss of hearing on both sides 1614.00; loss of hearing on one side only 143.00; loss of hearing which was sudden 111986.00; loss of hearing which was temporary 1619.00; MIXED CONDUCTIVE AND SENSORINEURAL HEARING LOSS 34077.00; NEURAL HEARING LOSS 37604.00; NOISE INDUCED HEARING LOSS 30788.00; Problems With Hearing 1003645.00; Problems With Hearing (on neurological exam) 11760.00; reported hearing problems using hearing aid both ears 127789.00; reported hearing problems using hearing aid right ear 127787.00; SENSORINEURAL HEARING LOSS 34075.00; SENSORINEURAL HEARING LOSS ASYMMETRICAL 311919.00; SENSORINEURAL HEARING LOSS BILATERAL 311925.00; SENSORINEURAL HEARING LOSS LEFT EAR 311924.00; SENSORINEURAL HEARING LOSS OF COMBINED TYPES 37606.00; SENSORINEURAL HEARING LOSS OF COMBINED TYPES BILATERAL 311922.00; SENSORINEURAL HEARING LOSS RIGHT EAR 311923.00; SENSORY HEARING LOSS 37603.00; SENSORY HEARING LOSS BILATERAL 311912.00; SENSORY HEARING LOSS UNILATERAL 312658.00; SPEECH AND LANGUAGE DEVELOPMENTAL DELAY DUE TO HEARING LOSS 312640.00; SUDDEN HEARING LOSS OF UNKNOWN ETIOLOGY 37597.00; total loss of hearing on both sides 1678.00

General notes Like "*Hearing*" Or Like "*Hear*" And Not Like 
History of ICD-9 codes falls

\begin{tabular}{|c|c|c|}
\hline & $\begin{array}{l}\text { Medcin } \\
\text { findings }\end{array}$ & $\begin{array}{l}\text { a fall 4363.00; a fall due to slipping, tripping, or stumbling } \\
\text { 124608.00; a fall from a bed 4955.00; a fall from a structure } \\
\text { 124407.00; a fall from furniture 120562.00; a fall from } \\
\text { stairs 4657.00; a fall into a hole } 120194.00 \text {; a fall, striking } \\
\text { an object 122430.00; Assess/Interv Future Risk Document } \\
\text { 2+ Falls In Past Year 303647.00; Ataxic Gait - Staggering } \\
\text { Or Falling To The Right 9038.00; fall due to ice and snow } \\
\text { 128644.00; fall in shower or empty bathtub 128697.00; fall } \\
\text { on same level from slipping, tripping and stumbling } \\
\text { 128645.00; INJURY DUE TO UNDETERMINED INTENT } \\
\text { FALL 95832.00; INJURY DUE TO UNDETERMINED } \\
\text { INTENT FALL HOUSE 212627.00; LATE EFFECTS OF } \\
\text { ACCIDENTAL FALL } 38136.00\end{array}$ \\
\hline & General notes & $\begin{array}{l}\text { Like "*fall*" And Not Like "*asleep*" And Not Like "*this } \\
\text { fall at*" And Not Like "*fallen asleep*" And Not Like } \\
\text { "*earlier this fall*" And Not Like "*last fall*" And Not Like } \\
\text { "*date falls*" And Not Like "*falls on Sun*" And Not Like } \\
\text { "*of last fall*" And Not Like "*filling fall*" And Not Like } \\
\text { "*in fall*" And Not Like "*hair fall*" And Not Like "*tooth } \\
\text { to fall*" And Not Like "*tonsils*" And Not Like "*going to } \\
\text { fall*" And Not Like "*cap fall*" And Not Like "*fall 20*" } \\
\text { And Not Like "*did not fall*" And Not Like "*preschool for } \\
\text { fall*" And Not Like "*this falls on*" And Not Like } \\
\text { "*fallopian*" And Not Like "*in the fall*" And Not Like } \\
\text { "*falls on a weekend" And Not Like "*falling asleep*" } \\
\text { And Not Like "*fall off on the*" And Not Like "*cancer last } \\
\text { fall*" And Not Like "*tsh is falling*" And Not Like "*f/p } \\
\text { on mobility*" And Not Like "*falling on a week" And Not } \\
\text { Like "*fall season*" And Not Like "*falling apart*" And } \\
\text { Not Like "*falls in this dosage*" And Not Like } \\
\text { "*falls rsik low*" Or Like "*fell*" }\end{array}$ \\
\hline \multirow{2}{*}{$\begin{array}{l}\text { Parkinson's } \\
\text { disease }\end{array}$} & ICD-9 codes & Parkinson's disease 332.0 \\
\hline & $\begin{array}{l}\text { Medcin } \\
\text { findings }\end{array}$ & $\begin{array}{l}\text { PARKINSON DISEASE 32004.00; PARKINSON } \\
\text { DISEASE W/ DEMENTIA W/O BEHAVIORAL } \\
\text { DISTURBANCE 312237.00; PARKINSON DISEASE }\end{array}$ \\
\hline
\end{tabular}

Accidental fall E880.0 - E888.9; Late effects of accidental fall E929.3; History of fall or at-risk for falling V15.88

a fall 4363.00; a fall due to slipping, tripping, or stumbling 124608.00; a fall from a bed 4955.00; a fall from a structure 124407.00; a fall from furniture 120562.00; a fall from (ans 4657.00 , a fall into a hole 120194.00, a fall, striking an object 122430.00, Assess/Interv Future Risk Document 2+ Falls In Past Year 303647.00; Ataxic Gait - Staggering Or Falling To The Right 9038.00; fall due to ice and snow 128644.00; fall in shower or empty bathtub 128697.00; fall on same level from slipping, tripping and stumbling 128645.00; INJURY DUE TO UNDETERMINED INTENT FALL 95832.00; INJURY DUE TO UNDETERMINED INTENT FALL HOUSE 212627.00; LATE EFFECTS OF

"*voices*" And Not Like "*test*" And Not Like "*exam*" And Not Like "*check*" And Not Like "*screen*" And Not Like "*inquiry*" And Not Like "*evaluation*" And Not Like "*black lung*" 
WITH DEMENTIA 38397.00

General notes Like "*Parkinson*" And Not Like "*Parkinsonism*" And Not Like "*Parkinsonian*" And Not Like "*Wolff*" And Not Like "*Not Positive*"

Poly- Medications Calculated based on current prescriptions for four or more pharmacy

Prescription Medications for antihypertensive

Prescription Medications for antiepileptic medications

Accuretic; Aldactazide; Aldoclor; Aldoclor-150; Aldoclor250; Aldoril 15; Aldoril 25; Aldoril D30; Aldoril D50; Aldoril; Aliskiren; Amiloride; Amlobenz; amlodipine; Amturnide; Apresazide; Atacand HCT; atenolol; atorvastatin; Avalide; azilsartan medoxomil; Azor; benazepril; bendroflumethiazide; Benicar HCT; BiDil; Bisoprolol; Caduet; candesartan; Capozide 25/15; Capozide 25/25; Capozide 50/15; Capozide 50/25; Capozide; captopril; chlorothiazide; Chlorthalidone ; Clorpres; Corzide 40/5; Corzide 80/5; Corzide; Demi-Regroton; deserpidine; Diltiazem; Diovan HCT; Diupres; Diupres-250; Diupres500; Diuretic Ap-Es; Dutoprol; Dyazide; Edarbyclor; Enalapril; Enduronyl; eprosartan; Esimil; Exforge HCT; Exforge; Felodipine; Fosinopril; guanethidine; hydralazine; Hydrap-ES; Hydra-Zide; hydrochlorothiazide; Hydropres; Hydropres-25; Hydropres-50; Hydroserpine; Hyzaar; Inderide; Irbesartan; Lexxel; Lisinopril; Lopressor HCT; losartan; Lotensin HCT; Lotrel; Maxzide; Maxzide-25; methyldopa; Metoprolol; Micardis HCT; Minizide; Moduretic 5-50; Moduretic; moexipril; Monopril HCT; Nadolol; Olmesartan; polythiazide; Prazosin; Prinzide; propranolol; quinapril; Quinaretic; Regroton; Renese-R; reserpine; Ser-Ap-Es; Serpazide; spironolactone; Tarka; Teczem; Tekamlo; Tekturna HCT; telmisartan; Tenoretic 100; Tenoretic 50; Tenoretic; Teveten HCT; Timolide 1025; Timolide; Timolol; trandolapril; triamterene; Tribenzor; Tri-Hydroserpine; Twynsta; Uni Serp; Uniretic; valsartan; Valturna; Vaseretic; Vaseretic 10-25; Vaseretic 5-12.5; Vaseretic; verapamil; Zestoretic; Ziac

Acetazolamide; Carbamazepine; Carbogen modified release; Clobazam; Clonazepam; Convulex; Desitrend; Diacomit; Diamox SR; Emeside; Epanutin; Epilim; Epilim Chrono; Epilim Chronosphere; Episenta prolonged release; Epival; Eslicarbazepine acetate; Ethosuximide; Frisium; Fycompa; Gabapentin; Gabitril; Inovelon; Keppra; Lacosamide; Lamictal; Lamotrigine; Levetiracetam; Lyrica; Neurontin; 
Prescription Medications for sedative

Use of walking aid/device

Vision ICD-9 codes impairment
Nitrazepam; Nootropil; Oxcarbazepine; Perampanel; Phenobarbital; Phenytoin; Phenytoin Sodium Flynn; Piracetam; Pregabalin; Primidone; Retigabine; Rivotril; Rufinamide; Sabril; Sodium valproate; Stiripentol; Tapclob; Tegretol; Tegretol Prolonged Release; Tiagabine; Topamax; Topiramate;Trileptal; Trobalt; Vigabatrin; Vimpat; Zarontin; Zebinix; Zonegran; Zonisamide

Adgan; Anergan 50; Antinaus 50; Aquachloral Supprettes; Atarax; Ativan; butabarbital; Butisol Sodium; chloral hydrate; Desyrel Dividose; Desyrel; dexmedetomidine; fentanyl; fospropofol; hydroxyzine; Hyzine; lorazepam; Lorazepam Intensol; Luminal; Lusedra; Mebaral; mephobarbital; Nembutal Sodium Nembutal; pentazocine; pentobarbital; Phenadoz; Phenergan; phenobarbital; Precedex; promethazine; Promethegan; secobarbital; Seconal Sodium; Seconal; Solfoton; Somnote; Sublimaze; Talwin; trazodone; Vistaril

General notes

Like "*wheeled walker*" Or Like "*wheel walker*" Or Like "*a walker*" Or Like "*using walker*" Or Like "*has walker*" Or Like "*new walker*" Or Like "*requested walker*" Or Like "*of walker*" Or Like "*about walker*" Or Like "*use walker*" Or Like "*uses walker*" Or Like "*give walker*" Or Like "*uses cane*" Or Like "*a cane*" Or Like "*has cane*" Or Like "*new cane*" Or Like "*used cane*" Or Like "*for cane*" Or Like "*of cane*" Or Like "*using cane*" Or Like "*requested cane*" Or Like "* give cane*" Or Like "*walking aid*" Or Like "*walking device*"

Blindness and low vision 369.0 - 369.9

Medcin findings
BINOCULAR VISION DISORDER 36625.00; blind spot (scotoma) 105.00; blurry vision 113.00; blurry vision as if looking through a glass of water 2016.00; blurry vision binocular 110203.00; blurry vision left 110202.00; blurry vision right 110201.00; COLOR BLINDNESS 30415.00; DAY BLINDNESS 318157.00; foggy vision 111364.00; foggy vision binocular 111367.00; foggy vision right 111365.00; headache preceded by double vision 68.00; headache preceded by loss of all vision in both eyes 66.00; headache preceded by loss of all vision in one eye (anopsia) 65.00; LATE CVD EFFECTS - VISION DISTURBANCES 272320.00; LEGALLY BLIND (USA DEFINITION) 36662.00; LEGALLY BLIND (USA DEFINITION) BOTH 
EYES 311746.00; LEGALLY BLIND (USA DEFINITION)

RIGHT EYE 311744.00; loss of part of field of vision 104.00; ONE EYE: PROFOUND IMPAIRMENT; OTHER

EYE: NEAR-NORMAL VISION 92938.00; ONEEYE:

TOTAL IMPAIRMENT; OTHER EYE: NORMAL

VISION 92933.00; Problems With Sight (on neurological exam) 11759.00; seeing insects at the edge of one's vision 1240.00; total loss of vision 1603.00; total vision loss left 2546.00; total vision loss unilaterally 1604.00;

TRAUMATIC BLINDNESS - LEFT EYE 39760.00; Vision

Assessment 6577.00; vision distortion 128.00; vision problems 111363.00; VISION SENSITIVITY

DEFICIENCY 335352.00; vision worsens during the day 281502.00; Visual Acuity - Cortical Blindness 6592.00; white / light spots in field of vision 111376.00; worsening distance and near vision 111313.00; worsening distance and near vision right 111847.00 ; worsening distance vision 2904.00; worsening distance vision left 111842.00;

worsening distance vision right 111841.00; worsening near vision 2905.00; worsening peripheral vision right 94.00; worsening vision 91.00; worsening vision occurring briefly (for a few minutes) 97.00; worsening vision progressing slowly 98.00; worsening vision right 102.00; worsening vision started suddenly 100.00 ; worsening vision sustained 111322.00; worsening vision worse in the morning 112172.00

General notes Like "*Blind*” Or Like "*Vision Imp*" Or Like "*Impaired Vision*" Or Like "*low vis*" Or Like "*vision*" Or Like "*sight*" And Not Like "*exam*" And Not Like "*milestone*" And Not Like "*guidance*" And Not Like "*supervision*" And Not Like "*oversight*" And Not Like

"*religious*" And Not Like "*test*" And Not Like "*provision*" And Not Like "*television*" And Not Like "*insight*" And Not Like *"Revision*" And Not Like "*20/_*" And Not Like "*plus_*" And Not Like "*not obscuring*" And Not Like "*confrontation*" 


\section{Appendix I: Expanded Falls Risk Factors, Locations of Data Extraction, and Coding}

\begin{tabular}{|c|c|c|}
\hline Factor & Location & Coding \\
\hline $\begin{array}{l}\text { Blood } \\
\text { pressure } \\
\text { systolic }\end{array}$ & Vitals & Systolic blood pressure value (mmHG) \\
\hline $\begin{array}{l}\text { Blood } \\
\text { pressure } \\
\text { diastolic }\end{array}$ & Vitals & Diastolic blood pressure value (mmHG) \\
\hline $\begin{array}{l}\text { Body mass } \\
\text { index }\end{array}$ & Vitals & Calculated body mass index \\
\hline \multirow[t]{2}{*}{$\begin{array}{l}\text { Diabetes } \\
\text { type I }\end{array}$} & ICD-9 codes & $\begin{array}{l}\text { Diabetes mellitus type I } 250.01 ; 250.03 ; 250.11 ; 250.13 \text {; } \\
250.21 ; 250.23 ; 250.31 ; 250.33 ; 250.41 ; 250.43\end{array}$ \\
\hline & $\begin{array}{l}\text { Medcin } \\
\text { findings }\end{array}$ & $\begin{array}{l}\text { DIAB W/ OPHTH MANIFESTATIONS TYPE } 1 \\
\text { UNCONTROLLED RIGHT EYE 277991.00; DIABET } \\
\text { HYPERGLYC HYPEROSMOLAR NONKETOTIC } \\
\text { STATE COMA (TYPE I) 92762.00; DIABETES } \\
\text { MELLITUS DIABETIC AUTONOMIC NEUROPATHY } \\
\text { TYPE } 1212787.00 ; \text { DIABETES MELLITUS DIABETIC } \\
\text { PERIPHERAL NEUROPATHY TYPE } 199839.00 ; \\
\text { DIABETES MELLITUS TYPE } 130481.00 ; \text { DIABETES } \\
\text { MELLITUS TYPE } 1 \text { - UNCONTROLLED 92759.00; } \\
\text { DIABETES MELLITUS TYPE } 1 \text { WITH COMPLICATION } \\
\text { 99851.00; DIABETES MELLITUS TYPE } 1 \text { WITH } \\
\text { COMPLICATION UNCONTROLLED 99853.00; } \\
\text { DIABETES MELLITUS TYPE 1 WITH } \\
\text { HYPERGLYCEMIA 315246.00; DIABETES MELLITUS } \\
\text { TYPE } 1 \text { WITH MANIFESTATIONS 99847.00; } \\
\text { DIABETES MELLITUS TYPE } 1 \text { WITH } \\
\text { MANIFESTATIONS UNCONTROLLED 99848.00; } \\
\text { DIABETES MELLITUS TYPE } 1 \text { WITH MULTIPLE } \\
\text { COMPLICATIONS 351497.00; DIABETES MELLITUS } \\
\text { TYPE } 1 \text { WITHOUT COMPLICATION 315582.00; } \\
\text { DIABETES MELLITUS WITH FOOT ULCER TYPE } 1 \\
\text { 276336.00; DIABETES MELLITUS WITH FOOT ULCER } \\
\text { TYPE 1 UNCONTROLLED 276337.00; DIABETES W/ } \\
\text { PERIPH CIRCULATORY DISORDER TYPE } 1 \\
\text { UNCONTROLLED 99846.00; DIABETES WITH } \\
\text { KETOACIDOSIS TYPE 1 99829.00; DIABETES WITH } \\
\text { KETOACIDOSIS TYPE 1 UNCONTROLLED 99830.00; } \\
\text { DIABETES WITH NEUROLOGICAL COMPLICATIONS }\end{array}$ \\
\hline
\end{tabular}


Diabetes type II
TYPE 1 276312.00; DIABETES WITH OPHTHALMIC MANIFESTATIONS TYPE 1 UNCONTROLLED 276308.00

General notes

Like "*Diabetes type $1 *$ " Or Like "*DM type $1 *$ " Or Like "*DM type I*" Or Like "*Diabetes type I**DM-1*" Or Like "*DM1*" Or Like "*Type $1 *$ " Or Like "*Type-1*" Or Like "*DMI*" OrLike "*DM-I*" Or Like "*Type-I*" Or Like "*Type I*" And Not Like "*Blood*" And Not Like "*Herpes*" And Not Like "*Imperfecta*" And Not Like "*Crystal*" And Not Like "*HSV*" And Not Like "** Genitals*" And Not "*type of Medica*" And Not Like "*typed*"

ICD-9 codes

Diabetes mellitus type II 250.00; 250.02; 250.10; 250.12;

$250.20 ; 250.22 ; 250.30 ; 250.32 ; 250.40 ; 250.42$

Medcin findings

DIAB W/ OPHTH MANIFESTATIONS TYPE 2 UNCONTROLLED BOTH EYES 277987.00; DIAB W/ OPHTH MANIFESTATIONS TYPE 2 UNCONTROLLED LEFT EYE 277986.00; DIAB W/ OPHTH MANIFESTATIONS TYPE 2 UNCONTROLLED RIGHT EYE 277985.00; DIABETES HYPERGLYCEMIC HYPEROSMOLAR NONKETOTIC STATE TYPE 2 99831.00; DIABETES MELLITUS DIABETIC AUTONOMIC NEUROPATHY TYPE 2 212789.00; DIABETES MELLITUS DIABETIC PERIPHERAL NEUROPATHY TYPE 2 99838.00; DIABETES MELLITUS TYPE 2 30480.00; DIABETES MELLITUS TYPE 2 - INSULIN-TREATED, NON-INSULIN DEPENDENT 350143.00; DIABETES MELLITUS TYPE 2 - UNCOMPLICATED, CONTROLLED 273144.00; DIABETES MELLITUS TYPE 2 - UNCOMPLICATED, UNCONTROLLED 92758.00; DIABETES MELLITUS TYPE 2 IN OBESE 350042.00; DIABETES MELLITUS TYPE 2 WITH COMPLICATION 99850.00; DIABETES MELLITUS TYPE 2 WITH COMPLICATION UNCONTROLLED 99852.00; DIABETES MELLITUS TYPE 2 WITH DIABETIC NEUROPATHIC ARTHROPATHY 315290.00; DIABETES MELLITUS TYPE 2 WITH GANGRENE 350059.00; DIABETES MELLITUS TYPE 2 WITH HYPERGLYCEMIA 315291.00; DIABETES MELLITUS TYPE 2 WITH HYPOGLYCEMIA 315292.00; DIABETES MELLITUS TYPE 2 WITH HYPOGLYCEMIA WITH COMA 315293.00; DIABETES MELLITUS TYPE 2 WITH MANIFESTATIONS 276315.00; DIABETES MELLITUS 
TYPE 2 WITH MANIFESTATIONS UNCONTROLLED 276316.00; DIABETES MELLITUS WITH FOOT ULCER TYPE 2 276338.00; DIABETES MELLITUS WITH FOOT ULCER TYPE 2 UNCONTROLLED 276339.00; DIABETES W/ NEUROLOGICAL COMPLICATIONS TYPE 2 UNCONTROLLED 276311.00;

General notes Like "*Diabetes type $2 *$ " Or Like "*DM type 2*" Or Like "*DM type II*" Or Like "*Diabetes type II**DM-2*" Or Like "*DM2*" Or Like "*Type $2 *$ " Or Like "*Type-2*" Or Like "*DMII*" Or Like "*DM-II*" Or Like "*Type-2*" Or Like "*Type II*" And Not Like "*Blood*" And Not Like “*Herpes*" And Not Like "*Imperfecta*" And Not Like "*Crystal" " And Not Like "*HSV*" And Not Like "Genitals*" And Not "*type of Medica*" And Not Like "*typed*"

Diabetic neuropathy
ICD-9 codes

Medcin findings

General notes

Diabetic
Diabetic neuropathy 357.2

CHRONIC PAINFUL DIABETIC NEUROPATHY 350370.00; DIABETES MELLITUS DIABETIC AUTONOMIC NEUROPATHY 30488.00; DIABETES MELLITUS DIABETIC AUTONOMIC NEUROPATHY TYPE 1 212787.00; DIABETES MELLITUS DIABETIC AUTONOMIC NEUROPATHY TYPE 2 212789.00; DIABETES MELLITUS DIABETIC AUTONOMIC NEUROPATHY UNCONTROLLED 92763.00; DIABETES MELLITUS DIABETIC MONONEUROPATHY SIMPLEX 350044.00; DIABETES MELLITUS DIABETIC PERIPHERAL NEUROPATHY 30487.00; DIABETES MELLITUS DIABETIC PERIPHERAL NEUROPATHY TYPE 1 99839.00; DIABETES MELLITUS DIABETIC PERIPHERAL NEUROPATHY TYPE 2 99838.00; DIABETES MELLITUS SECONDARY WITH DIABETIC NEUROPATHY 315150.00; DIABETES MELLITUS SECONDARY WITH PERIPHERAL NEUROPATHY 313960.00; DIABETES WITH DIABETIC NEUROPATHY 315314.00; DIABETES WITH DIABETIC POLYNEUROPATHY 315316.00; DIABETIC AUTONOMIC NEUROPATHY TYPE 1 UNCONTROLLED 212788.00; DIABETIC AUTONOMIC NEUROPATHY TYPE 2 UNCONTROLLED 212790.00

Like "*Neuropathy*" And Like "*Diab*"

Diabetic retinopathy $362.01-362.07$ 


\begin{tabular}{|c|c|c|}
\hline retinopathy & $\begin{array}{l}\text { Medcin } \\
\text { findings }\end{array}$ & $\begin{array}{l}\text { DIABETES MELLITUS SECONDARY WITH DIABETIC } \\
\text { RETINOPATHY 315139.00; DIABETES WITH } \\
\text { DIABETIC RETINOPATHY 315298.00; DIABETES } \\
\text { WITH DIABETIC RETINOPATHY PROLIFERATIVE } \\
\text { 315310.00; DIABETIC RETINOPATHY 98355.00; } \\
\text { DIABETIC RETINOPATHY NONPROLIFERATIVE } \\
\text { 30485.00; DIABETIC RETINOPATHY PRE- } \\
\text { PROLIFERATIVE 277921.00; DIABETIC } \\
\text { RETINOPATHY PRE-PROLIFERATIVE BOTH EYES } \\
\text { 277924.00; DIABETIC RETINOPATHY } \\
\text { PROLIFERATIVE 30486.00; DIABETIC RETINOPATHY } \\
\text { RETINAL MICROANEURYSMS BOTH EYES } \\
\text { 277966.00; DIABETIC RETINOPATHY TYPE } 2 \\
\text { 315211.00; RETINOPATHY 212800.00; RETINOPATHY } \\
\text { NONPROLIFERATIVE 210147.00; RETINOPATHY } \\
\text { NONPROLIFERATIVE BOTH EYES 277903.00; } \\
\text { RETINOPATHY NONPROLIFERATIVE LEFT EYE } \\
\text { 277902.00; TYPE 2 DIABETES WITH DIABETIC } \\
\text { RETINOPATHY 315265.00 }\end{array}$ \\
\hline & General notes & Like "*Retinopathy*" And Like "*Diab*" \\
\hline Epilepsy & $\begin{array}{l}\text { ICD-9 codes } \\
\text { Medcin } \\
\text { findings }\end{array}$ & $\begin{array}{l}\text { Epilepsy } 345.0 \text { - 345.91; V17.2 other neurological diseases } \\
\text { EPILEPSY 313582.00; EPILEPSY AND RECURRENT } \\
\text { SEIZURES 31974.00; EPILEPSY GENERALIZED } \\
\text { 335635.00; PYKNO-EPILEPSY WITH INTRACTABLE } \\
\text { SEIZURE 275268.00; SEIZURE DISORDER } \\
\text { GENERALIZED NONCONVULSIVE PYKNO-EPILEPSY } \\
\text { 275267.00 }\end{array}$ \\
\hline
\end{tabular}

General notes Like "*epilepsy*"

Essential ICD-9 codes Essential hypertension 401.0 - 401.9 hypertension

Medcin findings
ACP Staging Stage 1 Hypertension: 140-159/90-99 294917.00; ACP Staging Stage 2 Hypertension: Greater Than Or = 160/100 294918.00; ARTERIOLAR NEPHRITIS WITH HYPERTENSION 275572.00; BENIGN HYPERTENSION 350325.00; ESSENTIAL HYPERTENSION 33291.00; ESSENTIAL HYPERTENSION ACCELERATED 33289.00; 
ESSENTIAL HYPERTENSION BENIGN 34080.00;

ESSENTIAL HYPERTENSION MALIGNANT 33292.00;

HYPERTENSION (SYSTEMIC) 33288.00;

HYPERTENSION (SYSTEMIC) MALIGNANT

350241.00; HYPERTENSION DIASTOLIC ESSENTIAL

33290.00; HYPERTENSION SYSTOLIC 339874.00;

HYPERTENSION SYSTOLIC ESSENTIAL 33293.00;

SECONDARY HYPERTENSION 39910.00;

SECONDARY HYPERTENSION BENIGN 39911.00;

SECONDARY HYPERTENSION MALIGNANT 39912.00

General notes Like "*HTN*" Or Like "*Hyperten*" Or Like "*High

Blood Pressure*" Or Like "*High BP*" Or Like "*Elevated

BP*" Or Like "*Elevated Blood Pressure*" And Not Like

"*Hypertens heart*" And Not Like "*prehyperten*" And

Not Like "*hypertensive heart*" And Not Like

"*pregnancy*" And Not Like "*heavy pressure*" And Not

Like "*antihypertensive*" And Not Like "*ocular*" And

Not Like "*venous*" And Not Like "*eclampsic*" And Not

Like "*kidney*" And Not Like "*portal*" And Not Like

"*episode*" And Not Like "*intracerbral*" And Not Like

"*iatrogenic*" And Not Like "*renal*" And Not Like

"**enovascular*" And Not Like "**screening*" And Not

Like "*nephrosclerosis*" And Not Like "*pulmonary*" And

Not Like "*maternal*" And Not Like "*vascular*"

Vitals

Patients with last three blood pressure readings consistently greater than or equal to $140 / 90 \mathrm{mmHG}$

Ethnicity Demographics Patient ethnicity: Hispanic/Latino; Not Hispanic/Latino

Falls $\quad$ Medcin $\quad$ PT FALLS ASSESS-DOCD LE1/YR 1101F; PTFALLS

Assessment findings ASSESS-DOCD GE2>/YR 1100F

General notes Like "*fall*" And Like "*assess*"

Falls Medcin Anticipatory Guidance: Preventing Falls 71090.00; RN

Guidance findings Care: Monitoring Patient on Fall Precautions 76326.00

Height Vitals Height (inches)

Hypotension ICD-9 codes Hypotension 458

Medcin

findings

CHRONIC HYPOTENSION 38310.00; HYPOTENSION 38480.00; Hypotension 6058.00; HYPOTENSION

ORTHOSTATIC 'DELAYED' 213414.00;

IATROGENIC HYPOTENSION 38481.00; IATROGENIC

HYPOTENSION DRUG-INDUCED 95863.00; 
ORTHOSTATIC HYPOTENSION 38311.00; Orthostatic Hypotension 6059.00; ORTHOSTATIC HYPOTENSION IDIOPATHIC 30476.00

General notes Like "*hypotension*"

Vitals Patients with last three blood pressure readings consistently less than or equal to $90 / 60 \mathrm{mmHG}$

Insurance Demographics status

Muscle weakness

Osteoporosis

Prescription for dementia medication

Prescription Medications for diabetes type I medication
ICD-9 codes

Medcin

findings

General notes

ICD-9 codes

Medcin findings

General notes

Medications

Medications

Insurance source: MEDICAID; MEDICARE; PRIVATE INSURANCE; PUBLIC

Muscle weakness (generalized) 728.87

muscle weakness 281082.00; muscle weakness generalized 282527.00

Like "*Muscle*" and Like "*Weakness*" and Not Like "**if muscle*”

Osteoporosis $733.0-733.09$

OSTEOPOROSIS 30472.00; OSTEOPOROSIS DISUSE 30474.00; OSTEOPOROSIS DRUG-INDUCED 30475.00;

OSTEOPOROSIS IDIOPATHIC 34477.00;

OSTEOPOROSIS POSTMENOPAUSAL 30473.00; OSTEOPOROSIS SENILE 37653.00; OSTEOPOROSIS TRANSIENT, HIP 230088.00

Like "**steoporosis*" and Not Like osteoporosis*" and Not Like "*prevention of osteoporosis*"

ergoloid mesylates; ergoloid mesylates systemic; Haldol; Haldol Decanoate; haloperidol; haloperidol systemic; Hydergine

Apidra; Apidra Solostar; Exubera; Humalog; Humalog KwikPen; Humalog Mix 50/50; Humalog Mix 50/ 50 KwikPen; Humalog Mix 50 / 50 Pen; Humalog Mix 75 / 25; Humalog Mix 75 / 25 KwikPen; Humalog Mix 75 / 25 Pen; Humalog Pen; Humulin 50 / 50; Humulin 70 /30; Humulin 70 / 30 Pen; Humulin L; Humulin N; Humulin N Pen; Humulin R; Humulin R (Concentrated); Humulin U; Iletin II Regular Pork; Iletin Lente; insulin aspart; insulin
"*Dexascan normal*" and Not Like "*No 
aspart/insulin aspart protamine; insulin detemir; insulin glargine; insulin glulisine; insulin inhalation; insulin isophane; insulin isophane/insulin regular; Insulin Lente Pork; insulin lispro; insulin lispro/insulin lispro protamine; Insulin Purified Regular Pork; insulin regular; insulin zinc; insulin zinc extended; Lantus; Lantus OptiClik Cartridge; Lantus Solostar; Lente Iletin II; Levemir; liraglutide; Novolin 70 / 30; Novolin 70 / 30 Innolet; Novolin 70 / 30 PenFill; Novolin L; Novolin N; Novolin N Innolet; Novolin N PenFill; Novolin R; Novolin R Innolet; Novolin R PenFill; Novolog; NovoLog; FlexPen; NovoLog Mix 70 / 30; NovoLog Mix 70 / 30 FlexPen; Novolog Mix 70 / 30 PenFill; NovoLog PenFill; pramlintide; pramlintide amylin analogs; ReliOn / Novolin 70 / 30; ReliOn / Novolin R; Relion Novolin 70 / 30 Innolet; Relion Novolin N; Symlin; Symlin Pen; SymlinPen 120; SymlinPen 60; Velosulin BR; Victoza

Prescription Medications for diabetes type II medication acarbose; acetohexamide; ActoPlus Met; ActoPlus Met XR; Actos; albiglutide; alogliptin; alogliptin/metformin; alogliptin/pioglitazone; Amaryl; Apidra; Apidra Solostar; Avandamet; Avandaryl; Avandia; bromocriptine; Bydureon; Byetta; canagliflozin; chlorpropamide; chromium picolinate; colesevelam; Cr-GTF; CRM; Cycloset; dapagliflozin; DiaBeta; Diabinese; Duetact; Dymelor; exenatide; Exubera; Farxiga; Fortamet; glimepiride; glimepiride/pioglitazone; glimepiride/rosiglitazone; glipizide; GlipiZIDE XL; glipizide/metformin; Glucophage; Glucophage XR; Glucotrol; Glucotrol XL; Glucovance; Glumetza; glyburide; glyburide/metformin; Glycron; Glynase; Glynase PresTab; Glyset; Humalog; Humalog KwikPen; Humalog Mix 50 / 50; Humalog Mix 50/50 KwikPen; Humalog Mix 50 / 50 Pen; Humalog Mix 75 / 25; Humalog Mix 75 / 25 KwikPen; Humalog Mix 75 / 25 Pen; Humalog Pen; Humulin 50 / 50; Humulin 70 / 30; Humulin 70 / 30 Pen; Humulin L; Humulin N; Humulin N Pen; Humulin R; Humulin R (Concentrated) Humulin U; Iletin II Regular Pork; Iletin Lente; insulin aspart; insulin aspart/insulin aspart protamine; insulin detemir; insulin glargine; insulin glulisine; insulin inhalation, rapid acting; insulin isophane; insulin isophane/insulin regular; Insulin Lente Pork; insulin lispro; insulin lispro/insulin lispro protamine; Insulin Purified Regular Pork; insulin regular; insulin zinc; insulin zinc 
Prescription Medications

for anti-

epileptic

medication

\begin{tabular}{|c|c|}
\hline $\begin{array}{l}\text { Prescription } \\
\text { for } \\
\text { hypotension } \\
\text { medication }\end{array}$ & Medications \\
\hline $\begin{array}{l}\text { Prescription } \\
\text { for } \\
\text { osteoporosis } \\
\text { medication }\end{array}$ & Medications \\
\hline
\end{tabular}

extended; Invokana; Janumet; Janumet XR; Januvia; Jentadueto; Juvisync; Kazano; Kombiglyze XR; Lantus; Lantus OptiClik Cartridge; Lantus Solostar; Lente Iletin II; Levemir; linagliptin; linagliptin/metformin; liraglutide; Metaglip; metformin; metformin/pioglitazone; metformin/repaglinide; metformin/rosiglitazone; metformin/saxagliptin; metformin/sitagliptin; Micronase; miglitol; nateglinide; Nesina; Novolin 70 / 30; Novolin 70 / 30 Innolet; Novolin 70 / 30 PenFill; Novolin L; Novolin N; Novolin N Innolet; Novolin N PenFill; Novolin R; Novolin R Innolet; Novolin R PenFill; Novolog; NovoLog FlexPen; NovoLog Mix 70 / 30; NovoLog Mix 70 / 30 FlexPen; Novolog Mix 70 / 30 PenFill; NovoLog PenFill; Onglyza; Orinase; Oseni; pioglitazone; pramlintide; PrandiMet; Prandin; Precose; ReliOn / Novolin 70 / 30; ReliOn / Novolin R; Relion Novolin 70 / 30 Innolet; Relion Novolin $\mathrm{N}$; repaglinide; Rezulin; Riomet; rosiglitazone; saxagliptin; simvastatin/sitagliptin; sitagliptin; Starlix; Symlin; Symlin Pen; SymlinPen 120; SymlinPen 60; Tanzeum; tolazamide; tolbutamide; Tolinase; Tol-Tab; Tradjenta; troglitazone; Velosulin BR; Victoza; Welchol

acetazolamide; carbamazepine; Carbatrol; Cerebyx; Depakote; Depakote ER; Depakote Sprinkles; Diamox; Diamox Sequels; Dilantin; divalproex sodium; Epitol; ethotoin; ezogabine; Fanatrex; felbamate; Felbatol; fosphenytoin; Fycompa; gabapentin; Keppra; Keppra XR; levetiracetam; Lyrica; Mebaral; mephobarbital; Neurontin; Paradione; paramethadione; Peganone; perampanel; Phenytek; phenytoin; Phenytoin Sodium; Phenytoin Sodium, Prompt; Potiga; pregabalin; Sabril; Tegretol; Tegretol XR; Tridione; trimethadione; vigabatrin

droxidopa; Gilchew IR; Levophed; Levophed Bitartrate; Lusonal; midodrine; Nasop; Nasop12; norepinephrine; Northera; Orvaten; phenylephrine; Phenyl-T; ProAmatine; Ricobid-D; Vazculep

Aclasta; Actonel; Actonel with Calcium; Alcalak; alendronate; alendronate/cholecalciferol; Alora; Aquazide H; Atelvia; Binosto; Boniva; Calcarb; Calcarb with D; Calcet; Calci Mix; Calci-Chew; Calcio Del Mar; Calciquid; calcitonin; Calcitrate with D; Calcium 600 D; calcium carbonate; calcium carbonate/risedronate; Calcium Concentrate; calcium glubionate; calcium lactate; Calcium Liquid Softgel; Calcium Oyster Shell; calcium/vitamin d; Cal-Gest; Caltrate; Caltrate 600 with D Plus Soy; Caltrate 
Prescription Medications

for

Parkinson's

disease

medication

Prescription Medications

for

rheumatoid

arthritis

medication
600+D; Caltrate Colon Health; Caltrate Gummy Bites; Caltro with Vitamin D; Cenestin; Citracal + D; Citracal Calcium Gummies; Citracal Petites; Citracal Regular 250 mg + D; Citrus Calcium with Vitamin D; Climara; Clinagen LA 40; conjugated estrogens; Dep Gynogen; Depogen; DHT; DHT Intensol; Dical Captabs; Dical-D; Dicalphos plus D; Didronel; dihydrotachysterol; Dioval 40; DuraEstrin; Duragen; Enjuvia; Esclim; Esidrix; esterified estrogens; Estraderm; estradiol; Estradiol Patch; Estragyn LA 5; Estratab; Estra-V 40; Estro-Cyp; estropipate; EstroSpan 40; etidronate; Evista; Forteo; Fortical; Fosamax; Fosamax Plus D; Gynodiol; Gynogen LA 20; hydrochlorothiazide; HydroDIURIL; Hytakerol; ibandronate; Medidiol 10; Menaval-20; Menest; Menostar; Miacalcin; Miacalcin Nasal; Microzide; Neo-Calglucon; Nephro Calci; O-Cal-D; Ogen; Ogen 0.625; Ogen 1.25; Ogen 2.5; Ortho-Est; Os-Cal 500; Os-Cal $500+$ D; Os-Cal 500 + Extra D; Oysco 500; Oysco 500 with D; Oysco D; Oysco D with Calcium; Oyst Cal 500; Oyst-Cal-D; Oyster Cal; Oyster Calcium; Oyster Shell; Oyster Shell Calcium; Oyster Shell Calcium 500; Oyster Shell Calcium with Vitamin D; Oystercal-D; Oyster-D; Posture; Posture-D H / P; Premarin; Premarin Intravenous; Premphase; Prempro; Prolia; raloxifene; Reclast; Ridactate; Risacal-D; risedronate; teriparatide; UPCal D; Valergen; Vivelle; Vivelle-Dot; zoledronic acid; Zometa

Akineton; Akineton HCl; amantadine; Apokyn; apomorphine; Artane; Atamet; Azilect; belladonna; Belladonna Tincture; benztropine; biperiden; bromocriptine; carbidopa/entacapone/levodopa; carbidopa/levodopa; Cogentin; Comtan; Eldepryl; entacapone; Exelon; Kemadrin; Larodopa; levodopa; Mirapex; Mirapex ER; Neupro; Parcopa; Parlodel; pergolide; Permax; pramipexole; procyclidine; rasagiline; Requip; Requip Starter Kit; Requip $\mathrm{XL}$; rivastigmine; ropinirole; rotigotine; selegiline; Sinemet; Sinemet CR; Stalevo; Stalevo 100; Stalevo 125; Stalevo 150; Stalevo 200; Stalevo 50; Stalevo 75; Symmetrel; Tasmar; tolcapone; Trihexane; trihexyphenidyl; Zelapar

Absorbine Jr Extra Strength; Actemra; Acthar; Actiprofen; Actron; Addaprin; Advil; Advil Liqui-Gels; Aflaxen; A-G Profen; Aleve; A-Methapred; Amigesic; Analgesic Balm; Analgesic Balm Greaseless; Anaprox; Anaprox-DS; Anexsia; Ansaid; Arava; Aristocort; Aristocort For 
Injection; Arthricare Cream; Arthritis Pain; Arthritis Pain Formula; Arthrotec; Ascriptin Enteric; Aspercreme Cream; Aspergum; Aspirin Buffered; Aspirin Lite Coat; Aspiritab; Aurolate; Azasan; Azulfidine; Azulfidine EN-tabs; Banalg; Banalg Hospital Strength; Baycadron; Bayer Aspirin; Bayer Aspirin Extra Strength Plus; Bayer Women's Aspirin With Calcium; Bengay; BENGAY Arthritis; BENGAY Greaseless; BENGAY Original; BENGAY Ultra; BENGAY Vanishing Scent; Bextra; Boroleum; Buffered Aspirin; Bufferin; Bufferin Arthritis Strength; Bufferin Extra Strength; Castiva Cooling; Cataflam; Celebrex; Cimzia; Clinacort; Clinalog; Clinoril; Co-Gesic; Cold \& Hot Pain Relief; Comfort Pac with Naproxen; Cooling Gel; Cortone Acetate; Cuprimine; Daypro; Decadron; Deep Down Pain Relief; Deltasone; Depen; Depen Titratabs; Depmedalone; Depo-Medrol; Depopred; De-Sone LA; Dexacen-4;

Dexacort Phosphate in Turbinaire; Dexamethasone Intensol; Dexasone; Dexasone LA; Dexpak Taperpak; Disalcid;

Dolacet; Dolagesic; Dolobid; Duexis; Duraflex Comfort; Duralone; Easprin; EC-Naprosyn; Ecotrin; Ecotrin Adult Low Strength; Ecotrin Maximum Strength; Ecpirin; Empirin; Enbrel; Eucalyptamint; Exocaine Plus; Fasprin; Feldene; Flanax Pain Reliever; Flex-All 454; Flex-All 454 Maximum Strength; Flex-All 454 Ultra Plus; Genacote; Gengraf; Genpril; Gordogesic; GRX Analgesic Balm; H.P. Acthar Gel; Halfprin; Haltran; Heet Analgesic Liniment; Heet Triple Action; Humira; Hycet; Hydrocet; IBU; IBU200; Ibu-4; Ibu-6; Ibu-8; Ibu-Tab; Imuran; Indocin; Indocin IV; Indocin SR; Kenalog-10; Kenalog-40; Ken-Jec 40; Kineret; Leader Naproxen Sodium; Liquicet; Litecoat Aspirin; Lodine; Lodine XL; Lorcet 10 / 650; Lorcet Plus; Lortab; Maxidone; Meclomen; Medipred; Medi-Seltzer; Medralone; Medrol; Medrol Dosepak; Menthol C; Mentholatum Deep Heating; Mentholatum Pain Gel; Mentholatum Pain Patch; Methylcotol; MethylPREDNISolone Dose Pack; Meticorten; Midol Extended Relief; Midol IB; Minit Rub; Mobic; Morgidox; Motrin; Motrin IB; Muscle Rub; Myochrysine; Myoflex Cream; Nalfon; Naprelan; Naprosyn; Neoral; Nephro-Derm; Norco; Norwich Aspirin; Nuprin; Ocudox; Orencia; Orudis; 
Orudis KT; Oruvail; Otrexup; Pain Stick Arthritis Formula; Pain Stick Sports Formula; PainZone; Panalgesic Gold;

Penetran Pain Relieving; Plaquenil; Precise Pain Relieving; Predacorten; Prednicot; Prevacid NapraPAC; Prevacid NapraPAC 375; Prevacid NapraPAC 500; Proprinal; QProfen; Rasuvo; Rayos; Relafen; Remicade; Rheumatrex Dose Pack; Rhuli Gel; Ridaura; Rituxan; Salflex; Salonpas Pain Gel; Salonpas Pain Patch; Salonpas Pain Spray; Salsitab; Satogesic; Satogesic Hot Gel; Satogesic Pad; Simponi; Solganal; Solu-Medrol; Solurex; Solurex LA; Stagesic; Sterapred; Sulfazine; Theracodophen Low 90; Thera-Gesic Plus; Tolectin; Tolectin DS; Triamcot; TriBuffered Aspirin; U-Tri-Lone; Vaporizing Cold Rub; Vicodin ES; Vimovo; Voltaren; Voltaren-XR; Wintergreen Oil; Xeljanz; Ximino; Xodol; YSP Aspirin; Zamicet; Zema Pak; Zolvit; ZORprin; Zydone

\begin{tabular}{|c|c|c|}
\hline $\begin{array}{l}\text { Prescription } \\
\text { for vertigo } \\
\text { medication }\end{array}$ & Medications & $\begin{array}{l}\text { Adgan; Anergan 50; Antinaus 50; Antivert; Bonine; } \\
\text { diphenidol; diphenidol systemic; Dramamine II; Dramamine } \\
\text { Less Drowsy; D-Vert; Meclicot; meclizine; Meni-D; } \\
\text { Phenadoz; Phenergan; promethazine; Promethegan; Travel- } \\
\text { Ease; Vontrol }\end{array}$ \\
\hline ace & Demographics & $\begin{array}{l}\text { AMERICAN INDIAN OR ALASKA NATIVE; ASIAN; } \\
\text { BLACK OR AFRICAN AMERICAN; MULTIPLE } \\
\text { RACES; NATIVE HAWAIIAN; NATIVE HAWAIIAN OR } \\
\text { OTHER PACIFIC ISLANDER; OTHER PACIFIC } \\
\text { ISLANDER; UNREPORTED/REFUSED TO REPORT; } \\
\text { WHITE }\end{array}$ \\
\hline \multirow{2}{*}{$\begin{array}{l}\text { Rheumatoid } \\
\text { arthritis }\end{array}$} & ICD-9 codes & Rheumatoid arthritis 714.0 \\
\hline & $\begin{array}{l}\text { Medcin } \\
\text { findings }\end{array}$ & $\begin{array}{l}\text { INFLAMMATORY MYOPATHY SECONDARY TO } \\
\text { RHEUMATOID ARTHRITIS 95358.00; } \\
\text { POLYMYOSITIS IN RHEUMATOID ARTHRITIS } \\
\text { 91066.00; POLYNEUROPATHY SECONDARY TO } \\
\text { RHEUMATOID ARTHRITIS 95335.00; RHEUMATOID } \\
\text { ARTHRITIS 31844.00; RHEUMATOID ARTHRITIS } \\
\text { ANKLE 230186.00; RHEUMATOID ARTHRITIS ANKLE } \\
\text { LEFT 230189.00; RHEUMATOID ARTHRITIS ANKLE } \\
\text { LEFT TALONAVICULAR 230190.00; RHEUMATOID } \\
\text { ARTHRITIS ANKLE RIGHT 230187.00; RHEUMATOID } \\
\text { ARTHRITIS ANKLE RIGHT TALONAVICULAR } \\
\text { 230188.00; RHEUMATOID ARTHRITIS FELTY'S }\end{array}$ \\
\hline
\end{tabular}


SYNDROME 31845.00; RHEUMATOID ARTHRITIS

KNEE LEFT 230996.00; RHEUMATOID ARTHRITIS

NODULE NECROBIOTIC 33833.00; RHEUMATOID

ARTHRITIS RF POSITIVE 279141.00; RHEUMATOID

ARTHRITIS WRIST BILATERAL 230993.00

General notes Like "*rheumatoid arthritis*" And Not Like "*juvenile*"

And Not Like "*screen*" And Not Like "*exam*"

Stumble General notes Like "*stumble*"

Slip General notes

Like "*slip*" and Not Like "*order slip*" and Not Like

"*slipped disc*" and Not Like "*slip faxed*" and Not Like

"*work slip*" and Not Like "*slippage*" and not like

"*needs slip*" and Not Like "*slip given*" and Not Like

"*dyslipidemia*" and Not Like "*school slip*" and Not

Like "*slip for*" and Not Like "*lab slip*" and Not Like

"*packing slip*" and Not Like "* given slip*" and Not Like

"*referral slip*" and Not Like "* permission slip*"

Trip General notes

Like "*trip*" Or Like "*tripped*" And Not Like "*strip*"

And Not Like "*amitriptyline*" And Not Like "*triple*"

And Not Like "*tripple*" And Not Like "*school trip*" And

Not Like "*airplane trip*" And Not Like "*car trip*" And

Not Like "*Tripack*" And Not Like "*lipatripsey*" And

Not Like "*trip to*" And Not Like "*amitriptylline*" And

Not Like "*anitriptyline*" And Not Like "*trips*" And Not

Like "*going on a trip*" And Not Like "*planning on a

trip*" And Not Like "*amitriptalyine*" And Not Like

"*Triplix*" And Not Like "* trip back to*" And Not Like

"*Tripak*" And Not Like "*make a trip*" And Not Like

"*made a trip*" And Not Like "*making a trip*" And Not

Like "*amitriptylline*" And Not Like "*amytriptoline*"

And Not Like "*Amytriptyline*" And Not Like "*triptan*"

And Not Like "*Amitripytlkine*" And Not Like "*field

trip*" And Not Like "*amitriptylene*" And Not Like

"*lithotripsy*" And Not Like "*making another*" And Not

Like "*make another*" And Not Like "*take a trip*" 


\section{Appendix J: Falls Risk Factors, References, and Risk Categories}

\begin{tabular}{|c|c|c|}
\hline Factor & Reference & $\begin{array}{c}\text { Risk } \\
\text { category }\end{array}$ \\
\hline Age $>=65$ & $\begin{array}{l}\text { (Caton, Wiley, Zhao, Moran, \& Zapka, 2011; Larson \& } \\
\text { Bergmann, 2008; Malone, Vollbrecht, \& Burke, 2010; } \\
\text { McInnes, Seers, \& Tutton, 2011; Roberts, McKay, \& } \\
\text { Shaffer, 2008; Stalenhoef, Crebolder, Knottnerus, \& Van } \\
\text { Der Horst, 1997; Tremblay, Berndt, Luther, Foulis, \& } \\
\text { Frensh, 2009; Weber, White, \& McIlvried, 2005) }\end{array}$ & Biologic \\
\hline $\begin{array}{l}\text { Cognitive } \\
\text { impairment }\end{array}$ & $\begin{array}{l}\text { (Agency for Healthcare Research and Quality, 2006; } \\
\text { Carpenter C. R., 2009; Deandrea, et al., 2010; Flores, 2012; } \\
\text { Gerbier, et al., 2011; Harlein, Dassen, Halfens, \& Heinze, } \\
\text { 2009; Huang, Gau, Lin, \& Kernohan, 2003; Larson \& } \\
\text { Bergmann, 2008; Sirkin \& Rosner, 2009; Malone, } \\
\text { Vollbrecht, \& Burke, 2010; Melton, Horvat, \& Ray, 2011; } \\
\text { McInnes, Seers, \& Tutton, 2011; Stalenhoef, Crebolder, } \\
\text { Knottnerus, \& Van Der Horst, 1997; Tremblay, Berndt, } \\
\text { Luther, Foulis, \& Frensh, 2009; Weber, White, \& } \\
\text { McIlvried, 2005) }\end{array}$ & Biologic \\
\hline $\begin{array}{l}\text { Dizziness- } \\
\text { Vertigo }\end{array}$ & $\begin{array}{l}\text { (Deandrea, et al, 2010; Huang, Gau, Lin, \& Kernohan, } \\
\text { 2003; Murray, Hill, Phillips, \& Waterson, 2005; Myers, } \\
\text { 2003; Stalenhoef, Crebolder, Knottnerus, \& Van Der Horst, } \\
\text { 1997) }\end{array}$ & Biologic \\
\hline Fear of falling & (Deandrea, et al., 2010) & Biologic \\
\hline $\begin{array}{l}\text { Gait-Balance } \\
\text { impairment }\end{array}$ & $\begin{array}{l}\text { (Caton, Wiley, Zhao, Moran, \& Zapka, 2011; Deandrea et } \\
\text { al., 2010; Dhital \& Stanford, 2010; Flores, 2012; Ganz, } \\
\text { Bao, Shekelle, \& Rubenstein, 2007; Huang, Gau, Lin, \& } \\
\text { Kernohan, 2003; Larson \& Bergmann, 2008; McInnes, } \\
\text { Seers, \& Tutton, 2011; Melton, Horvat, \& Ray, 2011; } \\
\text { Murray, Hill, Phillips, \& Waterson, 2005; Rubenstein, } \\
\text { Powers, \& MacLean, 2001; Sirkin \& Rosner, 2009; } \\
\text { Stalenhoef, Crebolder, Knottnerus, \& Van Der Horst, 1997; } \\
\text { Tremblay, Berndt, Luther, Foulis, \& Frensh, 2009; Weber, } \\
\text { White, \& Mcllvried, 2005) }\end{array}$ & Biologic \\
\hline Gender (female) & (Deandrea, et al., 2010; Shanthi \& Krishnaswamy, 2005) & Biologic \\
\hline General notes & $\begin{array}{l}\text { (Chen, Hripcsak, \& Friedman, 2006; Friedman \& Hripcsak, } \\
\text { 1999; Gerbier, et al., 2011; Ware, Mullett, \& Jagannathan, } \\
\text { 2009) }\end{array}$ & $\begin{array}{l}\text { Biologic / } \\
\text { Behavioral }\end{array}$ \\
\hline
\end{tabular}


Get Up and Go

Test results

Hearing

impairment

History of falls (especially 1-2+

falls in past 12

months)

Total number of medications prescribed on a scheduled basis (i.e., total number of active medications )

$(>=4$ medications as an independent risk factor)

$\mathrm{Rx}$ for antihypertensive

Parkinson's disease

Rx for antiepileptic

$\mathrm{Rx}$ for sedative

(Carpenter C. R., 2009; Deandrea, et al., 2010; Hegeman, van den Bemt, Duysens, \& van Limbeek, 2009; Larson \& Bergmann, 2008; Shanthi \& Krishnaswamy, 2005; Sirkin \& Rosner, 2009; Stalenhoef, Crebolder, Knottnerus, \& Van Der Horst, 1997; Weber, White, \& McIlvried, 2005)

Use of walking (Deandrea, et al, 2010; Myers, 2003)

(Flores, 2012; Huang, Gau, Lin, \& Kernohan, 2003;

Biologic preventive services task force recommendation statement, 2012)

(Deandrea, et al., 2010; Myers, 2003)

Biologic

(Agency for Healthcare Research and Quality, 2006;

Carpenter, Scheatzle, D'Antonio, Ricci, \& Coben, 2009;

Carpenter C. R., 2009; Caton, Wiley, Zhao, Moran, \&

Zapka, 2011; Close, Hooper, Glucksman, Jackson, \& Swift, 2003; Deandrea, et al, 2010; Dhital \& Stanford, 2010;

Flores, 2012; Tremblay, Berndt, Luther, Foulis, \& Frensh, 2009)

(Caton, Wiley, Zhao, Moran, \& Zapka, 2011; Deandrea, et al., 2010; Flores, 2012; Larson \& Bergmann, 2008; Malone, Vollbrecht, \& Burke, 2010; Murray, Hill, Phillips, \& Waterson, 2005; Rubenstein, Powers, \& MacLean, 2001; Sirkin \& Rosner, 2009; Weber, White, \& McIlvried, 2005)

(Deandrea, et al., 2010; Hegeman, van den Bemt, Duysens, \& van Limbeek, 2009; Huang, Gau, Lin, \& Kernohan, 2003)

(Carpenter C. R., 2009; Deandrea, et al., 2010; Larson \& Bergmann, 2008; Stalenhoef, Crebolder, Knottnerus, \& Van Der Horst, 1997)

(Deandrea, et al., 2010)

Behavioral

Behavioral

Biologic

aid/device 
Vision

impairment
(Deandrea et al., 2010; Dhital \& Stanford, 2010; Flores, 2012; Harlein, Dassen, Halfens, \& Heinze, 2009; Huang, Gau, Lin, \& Kernohan, 2003; Larson \& Bergmann, 2008;

McInnes, Seers, \& Tutton, 2011; Melton, Horvat, \& Ray, 2011; Moyer, 2012; Murray, Hill, Phillips, \& Waterson, 2005; Myers, 2003; J. V. Odom, Odom, \& Leys, 2011; C. T. Ray \& Wolf, 2008; Rubenstein, Powers, \& MacLean, 2001; Salonen \& Kivela, 2012; Tremblay, Berndt, Luther, Foulis, \& Frensh, 2009; Weber, White, \& Mcllvried, 2005) 
Appendix K: Aim 3 Data Dictionary

\begin{tabular}{|c|c|c|c|c|}
\hline Variable name & $\begin{array}{l}\text { Variable } \\
\text { definition }\end{array}$ & $\begin{array}{l}\text { Data } \\
\text { type }\end{array}$ & $\begin{array}{l}\text { Modeling } \\
\text { type }\end{array}$ & Value labels \\
\hline Patient_ID & $\begin{array}{l}\text { Patient ID } \\
\text { (unique, } \\
\text { deidentified } \\
\text { patient identifier } \\
\text { linked with } \\
\text { clinic code) } \\
\text { Clinic code } \\
\text { (code for } \\
\text { location at } \\
\text { which patient is } \\
\text { seen, linked }\end{array}$ & Numeric & Nominal & \\
\hline clinic_code & $\begin{array}{l}\text { Patient_ID) } \\
\text { Age }\end{array}$ & Numeric & Nominal & . \\
\hline Age & (continuous) & Numeric & Ordinal & $\dot{0}=$ Female $1=$ \\
\hline Gender & Gender & Numeric & Nominal & $\begin{array}{l}\text { Male } \\
0=\text { White } ; 1=\end{array}$ \\
\hline Race & Race & Numeric & Nominal & $\begin{array}{l}\text { Non-White } \\
0=\text { Not } \\
\text { Hispanic/Latino } \\
; 1=\end{array}$ \\
\hline Ethnicity & $\begin{array}{l}\text { Ethnicity } \\
\text { Insurance }\end{array}$ & Numeric & Nominal & $\begin{array}{l}\text { Hispanic/Latino } \\
0=\text { Private; } 1=\end{array}$ \\
\hline Insurance_source & source & Numeric & Nominal & $\begin{array}{l}\text { Public } \\
0=\text { Non- }\end{array}$ \\
\hline Payor_category & $\begin{array}{l}\text { Insurance payor } \\
\text { category }\end{array}$ & Numeric & Nominal & $\begin{array}{l}\text { Managed care; } 1 \\
=\text { Managed care }\end{array}$ \\
\hline FallsAsmt & $\begin{array}{l}\text { Documented } \\
\text { falls assessment } \\
\text { Year in which } \\
\text { falls assessment } \\
\text { was last }\end{array}$ & Numeric & Nominal & $\begin{array}{l}0=\text { No falls } \\
\text { assessment; } 1= \\
\text { Falls assessment }\end{array}$ \\
\hline FallsAsmt_Year & documented & Numeric & Ordinal & $\dot{0}=$ No falls \\
\hline FallsGuidance_Year & $\begin{array}{l}\text { Documented } \\
\text { falls guidance } \\
\text { Year in which } \\
\text { falls guidance } \\
\text { was last } \\
\text { documented }\end{array}$ & Numeric & Nominal & $\begin{array}{l}\text { guidance; } 1= \\
\text { Falls guidance }\end{array}$ \\
\hline
\end{tabular}




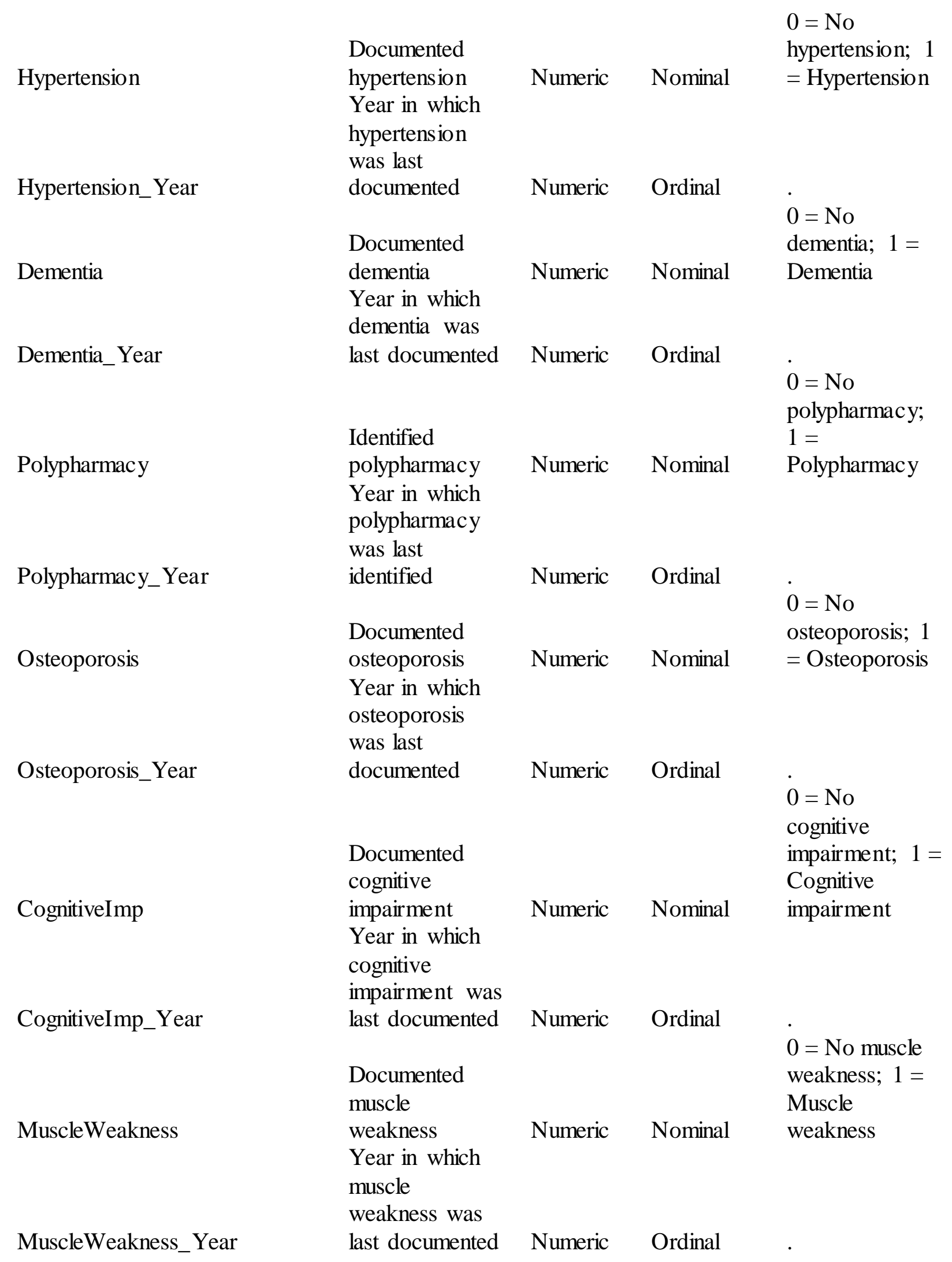




\begin{tabular}{|c|c|c|c|c|}
\hline HearingImp & $\begin{array}{l}\text { impairment } \\
\text { Year in which } \\
\text { hearing } \\
\text { impairment was }\end{array}$ & Numeric & Nominal & impairment \\
\hline HearingImp_Year & $\begin{array}{l}\text { last documented } \\
\text { Documented }\end{array}$ & Numeric & Ordinal & $\dot{0}=$ No arthritis \\
\hline Arthritis & $\begin{array}{l}\text { arthritis } \\
\text { Year in which } \\
\text { arthritis was last }\end{array}$ & Numeric & Nominal & $1=$ Arthritis \\
\hline Arthritis_Year & $\begin{array}{l}\text { Documented } \\
\text { dizziness/vertig }\end{array}$ & Numeric & Ordinal & $\begin{array}{l}\dot{0}=\text { No } \\
\text { dizzines s/vertig } \\
\text { o; } 1= \\
\text { Dizziness/Verti }\end{array}$ \\
\hline Dizziness-Vertigo & $\begin{array}{l}\text { o } \\
\text { Year in which } \\
\text { dizzines s/vertig } \\
\text { o was last }\end{array}$ & Numeric & Nominal & go \\
\hline Dizziness-Vertigo_Year & $\begin{array}{l}\text { documented } \\
\text { Documented }\end{array}$ & Numeric & Ordinal & $0=$ No DM- $1 ; 1$ \\
\hline DM-1 & $\begin{array}{l}\text { DM-1 } \\
\text { Year in which } \\
\text { DM-1 was last }\end{array}$ & Numeric & Nominal & $=\mathrm{DM}-1$ \\
\hline DM-1_Year & $\begin{array}{l}\text { documented } \\
\text { Documented }\end{array}$ & Numeric & Ordinal & $0=$ No DM- $2 ; 1$ \\
\hline DM-2 & $\begin{array}{l}\text { DM-2 } \\
\text { Year in which } \\
\text { DM-2 was last }\end{array}$ & Numeric & Nominal & $=\mathrm{DM}-2$ \\
\hline DM-2_Year & $\begin{array}{l}\text { documented } \\
\text { Documented } \\
\text { DM- }\end{array}$ & Numeric & Ordinal & $\begin{array}{l}0=\text { No DM- } \\
\text { Retinopathy; } 1 \\
=\text { DM- }\end{array}$ \\
\hline DM-Retinopathy & $\begin{array}{l}\text { Retinopathy } \\
\text { Year in which } \\
\text { DM- } \\
\text { Retinopathy } \\
\text { was last }\end{array}$ & Numeric & Nominal & Retinopathy \\
\hline DM-Retinopathy_Year & $\begin{array}{l}\text { documented } \\
\text { Documented }\end{array}$ & Numeric & Ordinal & $\begin{array}{l}0=\text { No DM- } \\
\text { Neuropathy; } 1=\end{array}$ \\
\hline DM-Neuropathy & DM-Neuropathy & Numeric & Nominal & DM-Neuropathy \\
\hline DM-Neuropathy_Year & $\begin{array}{l}\text { Year in which } \\
\text { DM-Neuropathy }\end{array}$ & Numeric & Ordinal & . \\
\hline
\end{tabular}


was last

documented

Epilepsy

Epilepsy_Year

Fall_Year

FearFalling_Year

Gait-BalanceImp

Gait-BalanceImp_Year

Hypotension

Hypotension_Year

VisionImp

VisionImp_Year
Documented

epilepsy

Year in which

epilepsy was

last documented

Documented

fall

Year in which a

fall was last

documented

Documented

fear of falling

Year in which

fear of falling

was last

documented

Documented

gait/balance

impairment

Year in which

gait/balance

impairment was

last documented

Documented

hypotension

Year in which

hypotension

was last

documented

Documented

vision

impairment

Year in which

vision

impairment was

last documented
$1=$ Epilepsy

Numeric Ordinal

$0=$ No history of fall; $1=$

Numeric Nominal History of fall

Numeric Ordinal

$0=$ No fear of falling; $1=$ Fear

Numeric Nominal of falling

Numeric Ordinal

$0=$ No

gait/balance

impairment; 1 = Gait/Balance

Numeric Nominal impairment

Numeric Ordinal

$0=\mathrm{No}$

Numeric Nominal = Hypotension

Numeric Ordinal

$0=$ No vision impairment; 1 = Vision

Numeric Nominal impairment

Numeric Ordinal 


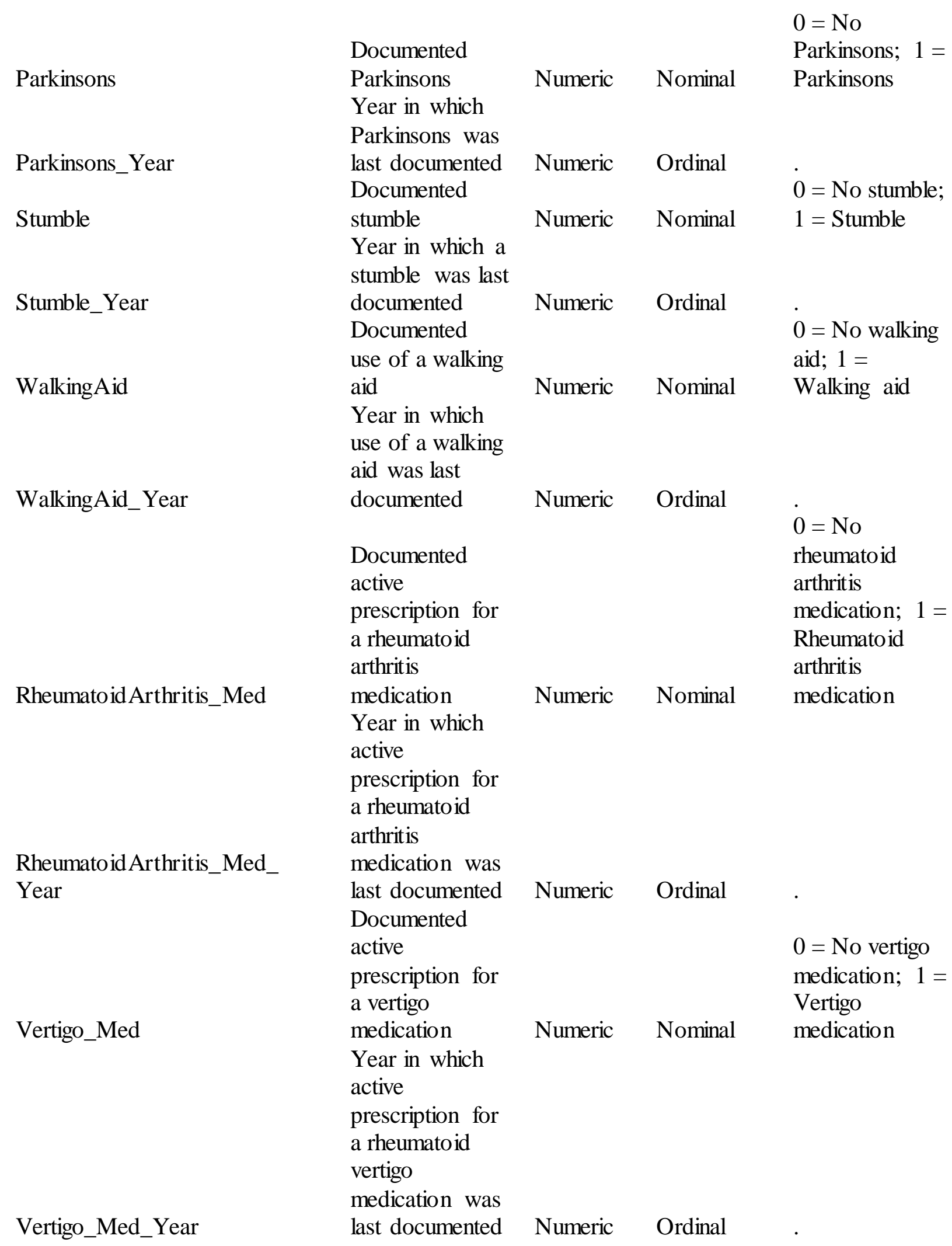


Sedative_Med

Sedative_Med_Year

AntiEpileptic_Med

AntiEpileptic_Med_Year

AntiHTN_Med

AntiHTN_Med_Year

Dementia_Med

Dementia_Med_Year
Documented

active

prescription for

a sedative

medication

Year in which

active

prescription for

a sedative

medication was

last documented

Documented

active

prescription for

an anti-epileptic

medication

Year in which

active

prescription for

an anti-epileptic

medication was

last documented

Documented

active

prescription for

an anti-

hypertensive

medication

Year in which

active

prescription for

an anti-

hypertensive

medication was

last documented

Documented

active

prescription for a dementia

medication

Year in which

active

prescription for a dementia

medication was

last documented
$0=$ No sedative medication; 1 = Sedative

Numeric Nominal medication

Numeric Ordinal

$0=$ No antiepileptic medication; 1 = Anti-epileptic

Numeric Nominal medication

Numeric Ordinal

$0=$ No antihypertensive medication; $1=$ Antihypertensive Numeric Nominal medication

Numeric Ordinal $0=\mathrm{No}$ dementia medication; 1 = Dementia Numeric Nominal medication

Numeric Ordinal 
DM-1_Med

DM-1_Med_Year

DM-2_Med

DM-2_Med_Year

Epilepsy_Med

Epilepsy_Med_Year

Hypotension_Med

Hypotension_Med_Year
Documented

active

prescription for

a DM-1

medication

Year in which

active

prescription for

a DM-1

medication was

last documented

Documented

active

prescription for

a DM-2

medication

Year in which

active

prescription for

a DM-2

medication was

last documented

Documented

active

prescription for

an epilepsy

medication

Year in which

active

prescription for

an epilepsy

medication was

last documented

Documented

active

prescription for a hypotension

medication

Year in which

active

prescription for

a hypotension

medication was

last documented
$0=$ No DM- 1

medication; $1=$

DM-1

Numeric Nominal medication

Numeric Ordinal

$0=$ No DM -2

medication; $1=$

DM-2

Numeric Nominal medication

Numeric Ordinal

$0=$ No epilepsy medication; $1=$ Epilepsy

Numeric Nominal medication

Numeric Ordinal

$0=\mathrm{No}$

hypotension

medication; 1 = Hypotension

Numeric Nominal medication 
Osteoporosis_Med

Osteoporosis_Med_Year

Parkinsons_Med

Parkinsons_Med_Year

Height

Height_Year

Height_DaysDiff

Weight

Weight_Year

Weight_DaysDiff
Documented

active

prescription for

an osteoporosis

medication

Year in which

active

prescription for

an osteoporosis

medication was

last documented

Documented

active

prescription for

a Parkinsons

medication

Year in which

active

prescription for

a Parkinsons

medication was

last documented

Last recorded

patient height

(in inches)

Last year in

which patient

height was

recorded

Time interval in

days between

date of first visit

and date of last

documentation

of patient height

Last recorded

patient weight

(in pounds)

Last year in

which patient

weight was

recorded

Time interval in days between

date of first visit

and date of last
$0=\mathrm{No}_{\mathrm{o}}$ osteoporosis

medication; $1=$ Osteoporosis

Numeric Nominal medication

Numeric Ordinal

$0=$ No

Parkinsons

medication; $1=$

Parkinsons

Numeric Nominal medication

Numeric Ordinal

Numeric Continuous .

Numeric Ordinal .

Numeric Ordinal

Numeric Continuous .

Numeric Ordinal

Numeric Ordinal 


\begin{tabular}{|c|c|c|c|}
\hline & $\begin{array}{l}\text { documentation } \\
\text { of patient } \\
\text { weight }\end{array}$ & & \\
\hline & $\begin{array}{l}\text { Last calculated } \\
\text { patient body }\end{array}$ & & \\
\hline BMI & $\begin{array}{l}\text { mass index } \\
\text { Last year in } \\
\text { which patient } \\
\text { body mass } \\
\text { index was }\end{array}$ & Numeric & Continuous \\
\hline BMI_Year & $\begin{array}{l}\text { calculated } \\
\text { Time interval in } \\
\text { days between } \\
\text { date of first visit } \\
\text { and date of last } \\
\text { calculation of } \\
\text { patient body }\end{array}$ & Numeric & Ordinal \\
\hline BMI_DaysDiff & $\begin{array}{l}\text { mass index } \\
\text { Last } \\
\text { documented } \\
\text { systolic blood }\end{array}$ & Numeric & Ordinal \\
\hline Systolic & $\begin{array}{l}\text { pressure reading } \\
\text { Last year in } \\
\text { which systolic } \\
\text { blood pressure } \\
\text { reading was }\end{array}$ & Numeric & Continuous \\
\hline Systolic_Year & $\begin{array}{l}\text { documented } \\
\text { Time interval in } \\
\text { days between } \\
\text { date of first visit } \\
\text { and date of last } \\
\text { documentation } \\
\text { of systolic blood }\end{array}$ & Numeric & Ordinal \\
\hline Systolic_DaysDiff & $\begin{array}{l}\text { pressure reading } \\
\text { Last } \\
\text { documented } \\
\text { diastolic blood }\end{array}$ & Numeric & Ordinal \\
\hline Diastolic & $\begin{array}{l}\text { pressure reading } \\
\text { Last year in } \\
\text { which diastolic } \\
\text { blood pressure } \\
\text { reading was }\end{array}$ & Numeric & Continuous \\
\hline Diastolic_Year & documented & Numeric & Ordinal \\
\hline
\end{tabular}


Diastolic_DaysDiff

Age_Cat_1

Age_Cat_2

BMI_Cat_1

Closest_BMI

Closest_Systolic

Closest_Diastolic
Time interval in days between date of first visit and date of last documentation of diastolic blood pressure reading using 65-84 and $85+$ age ranges Recoded age, using 65-74, 7584 , and $85+$ age ranges Recoded body mass index, using $<30$ and $>=30$ (obese) ranges

BMI measurement closest to the date of last documented fall. If no fall, then result = latest BMI Systolic blood pressure reading closest to the date of last documented fall. If no fall, then result $=$ latest systolic reading
Diastolic blood pressure reading closest to the date of last documented fall. If no fall, then result $=$ latest diastolic reading
Numeric Ordinal

Character Nominal .

Character Nominal .

$\begin{array}{ll}0 & =<30 ; 1= \\ \text { Character Nominal } \quad>=30\end{array}$

Numeric Continuous .

Numeric Continuous . 


\section{References}

Agency for Healthcare Research and Quality. (2006). Interdisciplinary teams perform daily reviews of real-time information to identify and address risk factors in older patients. Retrieved September 13, 2012, from www.innovations.ahrq.gov.aspx?id=3310

Amatayakul, M. (2005). EHR? Assess readiness first. Healthcare Financial Management, 59(5), 112-113.

American Geriatrics Society. (2001). Guideline for the prevention of falls in older persons. Journal of the American Geriatrics Society, 49(5), 664-672.

American Medical Association. (2012). Measure \#154 (NQF: 0101): falls: risk assessment. Retrieved from http://www.apta.org/uploadedFiles/APTAorg/Payment/Medicare/Pay_for_Performance/P QRS/2011/PQRSMeasure154.pdf

American Medical Association. (2013). Clinical decision support tools. Retrieved from http://www.ama-assn.org/ama/pub/physician-resources/practice-managementcenter/practice-operations/health-information-technology/clinical-decision-supporttools.page

American Medical Association and National Committee for Quality Assurance. (2010). Geriatric care falls risk assessment. Retrieved from http://www.rheumatology.org/Practice/Office/Pqri/2010_Measures/Measure_155/measur es154_155_specification/

Averbach Transcription. (2015). Averbach Transcription. Retrieved from https://avtranscription.com/

Baron, R. (2007). Quality improvement with an electronic health record: achievable, but not automatic. Annals of Internal Medicine, 147(8), 549-552.

Baron, R., Fabens, E., Schiffman, M., \& Wolf, E. (2005). Electronic health records: just around the corner? or over the cliff? Annals of Internal Medicine, 143, 222-226.

Bartholomew, L., Parcel, G., Kok, G., Gottlieb, N., \& Fernandez, M. (2011). Environmentoriented theories. In L. Bartholomew, G. Parcel, G. Kok, N. Gottlieb, \& M. Fernandez, Planning health promotion programs (3rd edition ed., pp. 113-167). San Francisco: Josey-Bass.

Bates, D. (2005). Physicians and ambulatory electronic health records. Health Affairs, 24(5), 1180-1189. 
Baus, A. D., Hendryx, M., \& Pollard, C. (2012). Identifying patients with hypertension: a case for auditing electronic health record data. Perspectives in Health Information Management, Spring, 1-15.

Baus, A., Wood, G., Pollard, C., Summerfield, B., \& White, E. (2013). Registry-based diabetes risk detection schema for the systematic identification of patients at risk for diabetes in West Virginia primary care centers. Perspectives in Health Information Management, Fall, 1-10.

Benin, A., Vitkauskas, G., Thornquist, E., Shapiro, E., Concato, J., Aslan, M., \& Krumholz, H. (2005). Validity of using an electronic medical record for assessing quality of care in an outpatient setting. Medical Care, 43(7), 691-698.

Berg, M. (1999). Patient care information systems and health care work: a sociotechnical approach. International Journal of Medical Informatics, 55(2), 87-101.

Berg, M. (2001). Implementing information systems in health care organizations: myths and challenges. International Journal of Medical Informatics, 64(2-3), 143-156.

Berg, P., Alessio, M., Mills, M., \& Tong, C. (1997). Circumstances and consequences of falls in independent community-dwelling older adults. Age and Ageing, 26(4), 261-268.

Berg, W. (1997). Circumstances and consequences of falls in independent community-dwelling older adults. Age and Ageing, 26(4), 261-268.

Blalock, J., Casteel, C., Roth, T., Ferreri, S., Demby, B., \& Shankar, V. (2010). Impact of enhanced pharmacologic care on the prevention of falls: a randomized controlled trial. The American Journal of Geriatric Pharmacotherapy, 8(5), 428-440.

Bohannon, R. (2006). Reference values for the timed up and go test: a descriptive meta-analysis. Journal of Geriatric Physical Therapy, 29(2), 64-68.

Bomgar. (2013). Secure remote desktop control with remote support software. Retrieved from http://www.bomgar.com

Botsis, T., Hartvigsen, G., Chen, F., \& Weng, C. (2010). Secondary use of EHR: data quality issues and informatics opportunities. Summit on Translational Bioinformatics, pp. 1-5.

Boyd, C., Darer, J., Boult, C., Fried, L., Boult, L., \& Wu, A. (2005). Clinical practice guidelines and quality of care for older patients with multiple comorbid diseases: implications for pay for performance. Journal of the American Medical Association, 294(6), 716-724.

Bradley, C., Penberthy, L., Devers, K., \& Holden, D. (2010). Health services research and data linkages: issues, methods, and directions for the future. Health Services Research, 45(5), 1468-1488. 
Bradley, E., Curry, L., \& Devers, K. (2007). Qualitative data analysis for health services research: developing taxonomy, themes, and theory. Health Services Research, 42(4), 1758-1772.

Bristol, N. (2005). The muddle of US electronic medical records. The Lancet, 365, 1610-1611.

Burton, L., Anderson, G., \& Kues, I. (2004). Using electronic health records to help coordinate care. The Milbank Quarterly, 82(3), 457-481.

Bush, R., Lord, E., \& Borrott, N. (2009). Diffusion of innovations: applying concepts in primary care and general practice. Healthy Communities Research Centre.

Business Intelligence Insider. (2014). Data integration techniques (ETL and data federation). Retrieved from http//bi-insider.com/portfolio/data-integration-techniques-etl-and-datafederation/

California HealthCare Foundation. (2002). Diffusion of innovation in health care. Institute for the Future, Oakland.

California Healthcare Foundation. (2003). Electronic medical records: lessons learned from small physician practices. San Francisco: California Healthcare Foundation.

Carpenter, C. R. (2009). Will my patient fall? Annals of Emergency Medicine, 53, 398-400.

Carpenter, C., Scheatzle, M., D'Antonio, J., Ricci, P., \& Coben, J. (2009). Identification of fall risk factors in older adult emergency department patients. Academic Emergency Medicine, 211-219.

Casteel, C., Blalock, J., Ferreri, S., Roth, T., \& Demby, B. (2011). Implementation of a community pharmacy-based falls prevention program. The American Journal of Geriatric Pharmacotherapy, 9(5), 310-319.

Caton, C., Wiley, M. K., Zhao, Y., Moran, W. P., \& Zapka, J. (2011). Improving internal medicine residents' falls assessment and evaluation: an interdisciplinary, multistrategy program. Journal of the American Geriatrics Society, 59, 1941-1946.

Centers for Disease Control and Prevention. (2013). STEADI (Stopping Elderly Accidents, Deaths \& Injuries) tool kit for health care providers. Retrieved from http://www.cdc.gov/homeand recreationalsafety/Falls/steadi/index.html

Centers for Disease Control and Prevention. (2014). Falls among older adults: an overview. Retrieved from http./www.cdc.gov/homeandrecreationalsafety/falls/adultfalls.html 
Chan, K., Fowles, J., \& Weiner, J. (2010). Electronic health records and the reliability and validity of quality measures: a review of the literature. Medical Care Research and Review, 67(5), 503-527.

Chen, E., Hripcsak, G., \& Friedman, C. (2006). Disseminating natural language processed clinical narratives. American Medical Informatics Association 2006 Symposium Proceedings, 126-130.

Christiadi. (2010). Why West Virginia population is aging faster than the U.S. West Virginia University, College of Business and Economics. Morgantown: Bureau of Business and Economic Research.

Cigolle, C., Ha, J., Min, L., Lee, P., Gure, T., Alexander, N., \& Blaum, C. (2015). The epidemiologic data on falls, 1998-2010: more older Americans report falling. The Journal of the American Medical Association. doi:10.1001/jamainternmed.2014.7533

Clarke, R. (1999). A primer in diffusion of innovations theory. Retrieved from http://www.rogerclarke.com/SOS/InnDiff.html

Close, J. C., Hooper, R., Glucksman, E., Jackson, S. H., \& Swift, C. G. (2003). Predictors of falls in a high risk population: results from the prevention of falls in the elderly trial (PROFET). Emergency Medicine Journal, 20, 421-425.

Coffey, A., \& Atkinson, P. (1996). Concepts and coding. In A. Coffey, \& P. Atkinson, Making sense of qualitative data (pp. 26-53). Newbury park: Sage Publications.

Damberg, L., Shortell, M., Raube, K., Gillies, R., Rittenhouse, D., McCurdy, K., \& Casalino, P. (2010). Relationship between quality improvement processes and clinical performance. The American Journal of Managed Care, 16(8), 601-606.

de Lusigna, S., \& van Weel, C. (2005). The use of routinely collected computer data for research in primary care: opportunities and challenges. Family Practice, 23(2), 253-263.

Dean, B., Lam, J., Natoli, J., Butler, Q., Aguilar, D., \& Nordyke, R. (2009). Use of electronic medical records for health outcomes research: a literature review. Medical Care Research and Review, 66(6), 611-638.

Deandrea, S., Lucenteforte, E., Bravi, F., Foscht, R., La Vecchta, C., \& Negri, E. (2010). Risk factors for falls in community-dwelling older people: a systematic review and metaanalysis. Epidemiology, 21, 658-668.

Dhital, A., \& Stanford, M. R. (2010). Visual loss and falls: a review. Eye, 24, 1437-1446.

Doolan, D., Bates, D., \& James, B. (2003). The use of computers for clinical care: a case series of advanced US cities. Journal of American Medical Informatics Association, 10, 94-107. 
Eliot \& Associates. (2005). Guidelines for conducting a focus group. Retrieved from http://assessment.aas.duke.edu/documents/How_to_Conduct_a_Focus_Group.pdf

Ethredge, L. (2010). Creating a high-performance system for comparative effectiveness research. Health Affairs, 29(10), 1761-1767.

Fabre, J. M. (2009). Identification of falls risk factors in community-dwelling older adults: validation of the comprehensive falls risk screening instrument.

Farley, T., Dalal, M., Mostashari, F., \& Frieden, T. (2010). Deaths preventable in the US by improvements in the use of clinical preventive services. American Journal of Preventive Medicine, 38(6), 600-609.

Fitzgerald, L., Ferlie, E., Wood, M., \& Hawkins, C. (2002). Interlocking interactions, the diffusion of innovations in health care. Human Relations, 55(12), 1429-1449.

Flores, E. K. (2012). Falls risk assessment and modification. Home Health Care Management \& Practice, 24(4), 198-204.

Forster, M., Brinkhof, M., Graber, C., Boulle, A., Sphor, M., Baelestre, E., \& Egger, M. (2008). Electronic medical record systems, data quality and loss to follow-up: survey of antiretroviral therapy programmes in resource-limited settings. Bulletin of the World Health Organization, 86(12), 939-947.

Freeman, E., Muñoz, B., Rubin, G., \& West, K. (2007). Visual field loss increases the risk of falls in older adults: the Salisbury eye evaluation. Investigative Opthalmology \& Visual Science, 48(10), 4445-4450.

Friedman, C., \& Hripcsak, G. (1999). Natural language processing and its future in medicine. Academic Medicine, 74(8), 890-895.

Gans, D., Kralewski, J., Hammons, T., \& Dowd, B. (2005). Medical groups' adoption of electronic health records and information systems. Health Affairs, 24(5), 1323-1333.

Ganz, D. A., Bao, Y., Shekelle, P. G., \& Rubenstein, L. Z. (2007). Will my patient fall? American Medical Association, 297(1), 77-86.

Gerbier, S., Yarovaya, O., Gicquel, Q., Millet, A., Smaldore, V., Pagliaroli, V., \& Metzger, M.H. (2011). Evaluation of natural language processing from emergency department computerized medical records for intra-hospital syndromic surveillance. BioMed Central Public Health, 11(50). doi:10.1186/1472-6947-11-50

Glanz, K. (2002). Perspectives on group, organization, and community interventions. In K. Glanz, B. Rimer, \& F. Lewis, Health behavior and health education (3rd edition ed., pp. 389-403). San Francisco: John Wiley \& Sons, Inc. 
Goldschmidt, P. (2005). HIT and MIS: implications of health information technology and medical information systems. Communication of the ACM, 48, pp. 69-74.

Greenhalgh, T., Stramer, K., Bratan, T., Byrne, E., Mohammad, Y., \& Russell, J. (2008). Introduction of shared electronic records: multi-site case study using diffusion of innovation theory. British Medical Journal, 337:a1786. doi:10.1136/bmj.a1786

Hanley, J., \& McNeil, B. (1983). A method of comparing the areas under receiver operating characteristic curves derived from the same cases. Radiology, 148(3), 839-843.

Hanna, K., Anderson, S., \& Maddox, S. (2005). Think research: using electronic medical records to bridge patient care and research. Washington, D.C.: The Center for Accelerating Medical Solutions.

Harlein, J., Dassen, T., Halfens, R. J., \& Heinze, C. (2009). Fall risk factors in older people with dementia or cognitive impairment: a systematic review. Journal of Advanced Nursing, 65(5), 922-933.

Harting, J., Rutten, G. M., Rutten, S. T., \& Kremers, S. P. (2009). A qualitative application of the diffusion of innovations theory to examine determinants of guideline adherence among physical therapists. Physical Therapy, 89(3), 221-232.

Hayden, S., Lupher, K., Caldwell, E., Miller, T., Kobayashi, M., Reynolds, R., \& Diggs, G. (2004). Strengths and needs assessment of older adults in the Denver metro area: technical report. Boulder: National Research Center. Retrieved from http://www.drcog.org/documents/Aging\%20Needs\%202.pdf

Hayrinen, K., Saranto, K., \& Nykanen, P. (2008). Definition, structure, content, use and impacts of electronic health records: a review of the research literature. International Journal of Medical Informatics, 77(5), 291-304.

Hazlehurst, B., Sittig, F., Stevens, J., Smith, S., Hollis, F., Vogt, M., \& Rigotti, A. (2005). Natural language processing in the electronic medical record: assessing clinician adherence to tobacco treatment guidelines. American Journal of Preventive Medicine, 29(5), 434-439.

Hegeman, J., van den Bemt, B. J., Duysens, J., \& van Limbeek, J. (2009). NSAIDS and the risk of accidental falls in the elderly: a systematic review. Drug Safety, 32(6), 489-498.

Heinze, T., Morsch, L., \& Holbrook, J. (2001). Mining free-text medical records. American Journal of Medical Informatics Association, Proc AMIA Symp, 254-258.

Hersh, R., Campbell, H., Evans, A., \& Brownlow, D. (1996). Empirical, automated vocabulary discovery using large text corpora and advanced natural language processing tools. American Medical Informatics Association, Proc AMIA Annual Fall Symp, 159-163. 
Hersh, W. (2002). Medical informatics: improving health care through information. Journal of the American Medical Association, 288(16), 1955-1958.

Himmelstein, D., \& Woodlander, S. (2005). Hope and hype: predicting the impact of electronic medical records. Health Affairs, 24(5), 1121-1123.

Hoff, G., Ottestad, P., Skaflotten, S., Bretthauer, M., \& Moritz, V. (2009). Quality assurance as an integrated part of the electronic medical record - a prototype applied for colonoscopy. Scandinavian Journal of Gastroenterology, 44, 1259-1265.

Hsieh, H., \& Shannon, S. (2005). Three approaches to qualitative content analysis. Qualitative Health Research, 15(9), 1277-1288.

Huang, H. C., Gau, M. L., Lin, W. C., \& Kernohan, G. (2003). Assessing risk of falling in older adults. Public Health Nursing, 20(5), 399-411.

Institute for Healthcare Improvement. (2013). IHI triple aim initiative. Retrieved from http:/www.ihi.org/offerings/Initiatives/TripleAim/Pages/default.aspx

Janetti, A. (2005). Case and grounded theory as qualitative research methods. Retrieved from http://www.redorbit.com/news/science/130594/case_and_grounded_theory_as_qualitativ e_research_methods

Johnson, T. (1985). Accidental falls among geriatric patients: can more be prevented? Journal of the American Medical Association, 77(8), 633-639.

Jones, T. S., Ghosh, T. S., Horn, K., Smith, J., \& Vogt, R. L. (2011). Primary care physicians perceptions and practices regarding fall prevention in adults 65 years and over. Accident Analysis and Prevention, 43, 1605-1609.

Kabiri, A., \& Chiadmi, D. (n.d.). A methodfor modelling and organizing ETL processes. EMI School.

Kaiser Family Foundation. (2013). State health facts - individual state profiles. Retrieved from http://kfforg/other/state-indicator/total-fqhes/?state=WV

Kaplan, B., \& Harris-Salamone, K. (2009). Health IT success and failure: recommendations from literature and an AMIA workshop. Journal of the American Medical Informatics Association, 16(3), 291-299.

Kaplowitz, M., \& Hoehn, J. (2001). Do focus groups and individual interviews reveal the same information for natural resource valuation? Ecological Economics, 36, 237-247. 
Kristianson, K., Ljunggren, H., \& Gustafsson, L. (2009). Data extraction from a semi-structured electronic medical record system for outpatients: a model to facilitate the access and use of data for quality control and research. Health Informatics Journal, 15(4), 305-319.

Krueger, R. (2002). Designing and conducting focus group interviews. St. Paul: University of Minnesota. Retrieved from http://www.eiu.edu/ ihec/Krueger-FocusGroupInterviews.pdf

Kuhn, K., \& Guise, D. (2001). From hospital information systems to health information systems. Methods of Medical Informatics, 40(4), 275-287.

Kukafka, R., Ancker, J., Chan, C., Chelico, J., Khan, S., Mortoti, S., \& Stephens, K. (2007). Redesigning electronic health record systems to support public health. Journal of Biomedical Informatics, 40(4), 398-409.

Larson, L., \& Bergmann, T. F. (2008). Taking on the fall: the etiology and prevention of falls in the elderly. Clinical Chiropractic, 11, 148-154.

Lewis, L., Moutoux, M., Slaughter, M., \& Bailey, P. (2004). Characteristics of individuals who fell while receiving home health services. Physical Therapy, 84(1), 23-32.

Lindsay, R., James, E., \& Kippen, S. (2004). The timed up and go test: unable to predict falls on the acute medical ward. Australian Journal of Physiotherapy, 50, 249-251.

Lorenzi, N., \& Riley, R. (2000). Managing change: an overview. Journal of American Medical Informatics Association, 72(2), 116-124.

Lorenzi, N., Riley, R., Blyth, A., Southon, G., \& Dixon, B. (1997). Antecedents of the people and organizational aspects of medical informatics. Journal of American Medical Informatics Association, 4(2), 79-93.

Makam, A. N., Nguyen, O. K., Moore, B., Ma, Y., \& Amarasingham, R. (2013). Identifying patients with diabetes and the earliest date of diagnosis in real time: an electronic heath record case-finding algorithm. BioMed Central Medical Informatics and Decision Making, 13(81), 1-7.

Malack, B. A. (2012). HIMSS topical review. Retrieved from Best practices of clinical decision support:

http://www.himss.org/files/HIMSSorg/content/files/BestPracticesC linicalDecisionS uppor t.pdf

Malone, M. L., Vollbrecht, M. S., \& Burke, L. (2010). Acute care for elders (ACE) tracker and e-geriatrician: methods to disseminate ACE concepts to hospitals with no geriatricians on staff. Journal of the American Geriatrics Society, 58, 161-167. 
Maurer, S., Burcham, J., \& Cheng., H. (2004). Diabetes mellitus is associated with an increased risk of falls in elderly residents of a long-term care facility. The Journals of Gerontology, 60(9), 1157-1162.

Maxwell-Downing, D. (2011). Data quality and the electronic health record (EHR). Association of periOperative Registered Nurses Journal, 94(6), 603-631.

May, J. (2005). The electronic medical record: a valuable partner. The Journal of Medical Practice Management, 21(2), 100-102.

McDonald, C. (1997). The barriers to electronic medical record systems and how to overcome them. Journal of American Medical Informatics Association, 4(3), 213-221.

McInnes, E., Seers, K., \& Tutton, L. (2011). Older people's views in relation to risk of falling and need for intervention: a meta-ethnography. Journal of Advanced Nursing, 67(12), 2525-2536.

Melton, F., Horvat, M., \& Ray, C. (2011). Intrinsic and functional components of falls risk in older adults with visual impairments. Insight: Research and Impact in Visual Impairment and Blindness, 4(2), 66-73.

Mendes, R., \& Rodrigues, P. (2011). Main barriers for quality data collection in EHR: a review. Health Informatics 2011 - International Conference on Health Informatics, (pp. 451454).

Meystre, S., \& Haug, P. (2005). Automation of a problem list using natural language processing. BioMed Central Medical Informatics and Decision Making, 5(30).

Meystre, S., \& Haug, P. (2006). Improving the sensitivity of the problem list in an intensive case unit by using natural language processing. American Medical Informatics Association, Proc AMIA Symp, 554-558.

Michael, Y., Whitlock, E., Kin, J., Fu, R., O'Connor, E., \& Gold, R. (2010). Primary CareRelevant Interventions to Prevent Falling in OlderAdults: A Systematic Evidence Review for the U.S. Preventive Task Force. Annals of Internal Medicine, 815-825.

Milery, M., \& Kukafka, R. (2010). Health information technology and quality of care: strategies for reducing disparities in underresourced settings. Medical Care Research and Review, 67(5), 268S-298S.

Miles, M. B., \& Huberman, A. M. (1994). Qualitative data analysis: an expanded sourcebook (2nd edition). London: Sage Publications, Inc.

Miller, R., \& Sim, I. (2004). Physicians' use of electronic medical records: barriers and solutions. Health Affairs, 23(2), 116-126. 
Moyer, V. (2012). Prevention of falls in community-dwelling older adults: US Preventive Services Task Force recommendation statement. Annals of Internal Medicine, 157, 197204.

Murphy, J. (2010). The journey to meaningful use of electronic health records. Nursing Economics, 28(4), 283-286.

Murray, K. J., Hill, K., Phillips, B., \& Waterson, J. (2005). A pilot study of falls risk and vestibular dysfunction in older fallers presenting to hospital emergency departments. Disability and Rehabilitation, 27(9), 499-506.

Myers, H. (2003). Hospital fall risk assessment tools: a critique of the literature. International Journal of Nursing Practice, 9, 223-235.

Nagle, B., \& Williams, N. (n.d.). Methodology brief: introduction to focus groups. Center for Assessment, Planning \& Accountability.

Nahm, M., Pieper, C., \& Cunningham, M. (2008). Quantifying data quality for clinical trials using electronic data capture. PLoS One, 3(8), e3049.

National Committee for Quality Assurance. (2009). Quality profiles: focus on supporting quality improvement through the use of health information technology. National Committee for Quality Assurance and Pfizer.

National Committee for Quality Assurance. (2013). Start to finish: patient-centered medical home $(P C M H)$ recognition. Retrieved from http://www.ncqa.org/Programs/Recognition/PatientCenteredMedicalHomePCMH.aspx

Nichols, G. A., Desai, J., Lafata, J. E., Lawrence, J. M., O’Connor, P. J., Pathak, R. D., \& Waitzfelder, B. (2012). Construction of a multisite datalink using electronic health records for the identification, surveillance, prevention, and management of diabetes mellitus: the SUPREME-DM project. Preventing Chronic Disease, 110311.

NVivo. (2015). NVivo software for qualitative research. Retrieved from http://www.qsrinternational.com/default.aspx

O'Carroll, P., Yasnoff, W., Ward, M., Ripp, L., \& Martin, E. (2003). Public health informatics and information systems. New York: Springer-Verlag New York, Inc.

Odom, J. V., Odom, C. V., \& Leys, M. J. (2011). Does improving vision reduce the risk of falls? A review. Insight: Research and Impact in Visual Impairment and Blindness, 4(2), 92-99.

Office of the National Coordinator for Health Information Technology. (2015). EHR incentives and certification. Retrieved from http://www.healthit.gov/providers-professionals/howattain-meaningful-use 
Office of the National Coordinator for Health Information Technology. (2015). Health IT Dashboard. Retrieved from Medicare professional stage 1 meaningful use attestation: http://dashboard.healthit.gov/index.php

Okun, S., McGraw, D., Stang, P., Larson, E., Goldmann, D., Kupersmith, J., \& Murray, M. (2013). Making the case for continuous learning from routinely collected data. Washington, D.C.: Institute of Medicine of the National Academies. Retrieved from http://www.iom.edu/makingthecase

Pandza, H. (2009). Minimal data sets for electronic medical records in hospital settings. Acta Informatica Medica, 17(2), 71-73.

Persell, S., Kho, A., Thompson, J., \& Baker, D. (2009). Improving hypertension quality measurement using electronic heath records. Medical Care, 47(4), 388-394.

Piva, S., Fitzgerald, G., Irrgang, J., Bouzubar, F., \& Starz, T. (2004). Get up and go test in patients with knee osteoarthritis. Archives of Physical Medicine and Rehabilitation, 85, 284-289.

Podsiadlo, D., \& Richardson, S. (1991). The timed "Up \& Go": a test of basic functional mobility for frail elderly persons. Journal of the American Geriatrics Society, 39, 142148.

Poses, R. M. (1999). One size does not fit all: questions to answer before intervening to change physician behavior. Joint Commission Journal on Quality Improvement, 25, 486-495.

Prevention of falls in community-dwelling older adults: US preventive services task force recommendation statement. (2012). Annals of Internal Medicine, 157(3), 197-204.

Prine, S. (1998). Working toward a design for qualitative research. In A. Teppo, Qualitative methods in mathematics education (pp. 79-97). Reston: The National Council of Teachers of Mathematics, Inc.

Ray, C. T., \& Wolf, S. L. (2008). Review of intrinsic factors related to fall risk in individuals with visual impairments. Journal of Rehabilitation Research \& Development, 45(8), 1117-1124.

Richardson, R., Hicks, J., \& Walker, B. (2002). Falls in rural elders: an empirical study of risk factors. Journal of the American Board of Family Practice, 15(3), 178-182.

Roberts, D. C., McKay, M. P., \& Shaffer, A. (2008). Increasing rates of emergency department visits for elderly patients in the united states, 1990 to 2003. Annals of Emergency Medicine, 51(6), 769-774.

Rogers, E. (1983). Diffusion of innovations. New York: Free Press. 
Romano, M., \& Stafford, R. (2011). Electronic health records and clinical decision support systems. Archives of Internal Medicine, 171(10), 897-903.

Rose, A., Schnipper, J., Park, E., Poon, E., Li, Q., \& Middleton, B. (2005). Using qualitive studies to improve the usability of an EMR. Journal of Biomedical Informatics, 38, 5160.

Rubenstein, L. Z., Powers, C. M., \& MacLean, C. H. (2001). Quality indicators for the management and prevention of falls and mobility problems in vulnerable elders. Annals of Internal Medicine, 135(8), 686-693.

Rural Assistance Center. (2013). FQHC frequently asked questions. Retrieved from http://www.raconline.org/topic s/clinics/fqhcfaq.php\#whatis

Salonen, L., \& Kivela, S. L. (2012). Eye diseases and impaired vision as possible risk factors for recurrent falls in the aged: a systematic review. Current Gerontology and Geriatrics Research, 2-10.

Sanson-Fisher, R. W. (2004). Diffusion of innovation theory for clinical change. Adopting Best Evidence in Practice, 180, S55-S56.

SAP Business Objects. (2013). SAP business management software solutions, applications and services. Retrieved from http://www.sap.com/index.epx

SAS. (2013). SAS business analytics and business intelligence software. Retrieved from http://www.sas.com

Satinsky, M. (2004). EHR: questions come before answers. Review of Opthalmology, 67-72.

Shanthi, G. S., \& Krishnaswamy, B. (2005). Risk factors for falls in elderly. Journal of the Indian Academy of Geriatrics, 2, 57-60.

Shires, D. A., Stange, K. C., Divine, G., Ratliff, S., Vashi, R., Tai-Seale, M., \& Lafata, J. E. (2012). Prioritization of evidence-based preventive health services during periodic health examinations. American Journal of Preventive Medicine, 42(2), 164-173.

Shumway-Cook, A., Brauer, S., \& Woollacott, M. (2000). Predicting the probability for falls in community-dwelling older adults using the timed up \& go test. Physical Therapy, 80(9), 896-903.

Siegler, L. (2010). The evolving medical record. Annals of Internal Medicine, 153(10), 671-677.

Siggeirsdóttir, K., Akranes, J., Jónsson Jr., H., \& Iwarsson, S. (2002). The timed 'up \& go' is dependent on chair type. Clinical Rehabilitation, 609-616. 
Simon, J., Rundall, T., \& Shortell, S. (2005). Drivers of electronic medical record adoption among medical providers. Joint Commission on Accreditation of Healthcare Organizations, 31(11), 631-639.

Sirkin, A. J., \& Rosner, N. G. (2009). Hypertensive management in the elderly population at risk for falls. Journal of the American Academy of Nurse Practitioners, 21, 402-408.

Stalenhoef, P. A., Crebolder, H. F., Knottnerus, A., \& Van Der Horst, F. G. (1997). Incidence, risk factors and consequences of falls among elderly subjects living in the community: a criteria-based analysis. European Journal of Public Health, 3, 328-334.

Stevens, J. A., \& Schuster, R. J. (2013). The stopping elderly accidents, deaths, \& injuries (STEADI) tool kit for health care providers. Centers for Disease Control and Prevention.

Stokes, M. E., Davis, C. S., \& Koch, G. G. (2012). Categocial data analysis using SAS. (T. edition, Ed.) Cary: SAS Institute, Inc.

Strauss, A., \& Corbin, J. (1994). Grounded theory methodology: an overview. In N. Denzin, \& Y. Lincoln, Handbook of qualitative research (pp. 1-18). London: Sage Publications.

SuccessEHS. (2013). SuccessEHS EHR and practice management solutions. Retrieved from http://www.successehs.com

Taylor, R., Bower, A., Girosl, F., Bigelow, J., Fonkych, K., \& Hills tead, R. (2005). Promoting health information technology: is there a case for more-aggressive government action? Health Affairs, 24(5), 1234-1245.

Terry, A., Chevendra, V., Think, A., Stewart, M., Marshall, J., \& Cejic, S. (2010). Using your electronic medical record for research: a primer for avoiding pitfalls. Family Practice, 27, 121-126.

Tolar, M., \& Balka, E. (2011). Beyond individual patient care: enhanced use of EHR data in a primary care setting. International Perspectives in Health Informatics, 164, 143-147.

Tremblay, M. C., Berndt, D. J., Luther, S. L., Foulis, P. R., \& Frensh, D. D. (2009). Identifying fall-related injuries: text mining the electronic medical record. Information Technology \& Management, 10, 253-265.

Twohig, P. L., \& Putnam, W. (2002). Group interviews in primary care research: advancing the state of the art or ritualized research? Family Practice, 19(3), 278-284.

US Census Bureau. (2012). State \& County QuickFacts. Retrieved from West Virginia: http:/quickfacts.census.gov/qfd/states/54000.html 
US Department of Health \& Human Services. (2014). Health information privacy. Retrieved from Guidance regarding methods for de-identification of protected health information in accordance with the Health Insurance Portability and Accountability Act (HIPAA) privacy rule: http://www.hhs.gov/ocr/privacy/hipaa/understanding/coveredentities/Deidentification/guidance.html

US Department of Health and Human Services - Health Resources and Services Administration. (2013). 2013 health center data - West Virginia program grantee data. Retrieved from http://bphc.hrsa.gov/uds/datacenter.aspx? year=2013\&state=WV

US Department of Health and Human Services. (2013). 2020 topice \& objectives: older adults. Retrieved from http:/healthypeople.gov/2020/topicsobjectives2020/objectiveslist.aspx?topic Id=31

Villaveces, A., Mutter, R., Owens, P., \& Barrett, M. (2013). Causes of injuries treated in the emergency department, 2010. Rockville, MD: Agency for Healthcare Research and Quality. Retrieved from http://www.hcup-us.ahrq.gov/reports/statbriefs/sb156.pdf

Vishwanath, A., Singh, S., \& Winkelstein, P. (2010). The impact of electronic medical record systems on outpatient workflows: a longitudinal evaluation of its workflow effects. International Journal of Medical Informatics, 79, 778-791.

Wagner, K., Lee, F., White, A., Ward, D., \& Ornstein, S. (2000). Impact of electronic medical record system on community-based primary care clinics. The Journal of the American Board of Family Practice, 13(5), 338-348.

Walsh, S. (2004). The clinician's perspective on electronic health records and how they can affect patient care. British Medical Journal, 328, 1184.

Ware, H., Mullett, C., \& Jagannathan, V. (2009). Natural language processing framework to assess clinical conditions. Journal of the American Medical Informatics Association, $16(4), 585-589$.

Weber, V., White, A., \& McIlvried, R. (2005). An electronic medical record (EMR)-based intervention to reduce polypharmacy and falls in an ambulatory elderly population. ournal of General Internal Medicine, 23(4), 399-404.

Weiner, M. G., Lyman, J., Murphy, S., \& Weiner, M. (2007). Electronic health records: highquality electronic data for higher-quality clinical research. Informatics in Primary Care, $15,121-127$.

Wenger, N. S., Solomon, D. H., Roth, C. P., MacLean, C. H., Saliba, D., Kamberg, C. J., \& Shekelle, P. G. (2003). The quality of medical care provided to vulnerable communitydwelling older patients. Annals of Internal Medicine, 139, 740-747. 
West Virginia Bureau for Public Health. (2008). Healthy people 2010: prevention map for the future. Retrieved from http://www.wvdhhr.org/bph/hp2010/

West Virginia University Office of Health Services Research. (2013). Chronic Disease Electronic Management System. Retrieved from http://publichealth.hsc.wvu.edu/ohsr/Services/Chronic-Disease-Electronic-ManagementSystem-(CDEM

Whiteman, C., Davidov, D., Tadros, A., \& D'Angelo, J. (2012). Falls and dilemmas in injury prevention in older West Virginians. The West Virginia Medical Journal, 108(3), 14-20.

Wrightson, W. (2010). A comparasion of elecetronic and handwritten anaesthetic records for completness of information. Anaesthesia and Intensive Care, 38(6), 1052-1058. 